VENTILATION DURING SLEEP ONSET 


\title{
VENTILATION DURING SLEEP ONSET
}

\author{
by \\ Michael \\ Ian M. Colrain B.Sc.(Hons.) \\ in the Department of Psychology
}

Submitted in fulfilment of the requirements

of the degree of Doctor of Philosophy

University of Tasmania

January, 1988. 
I certify that this thesis contains no material which has been accepted for the award of any other higher degree or graduate diploma in any university, and that to the best of my knowledge and belief the thesis contains no copy or paraphrase of material previously published or written by another person, except where due reference is made in the text of the thesis.

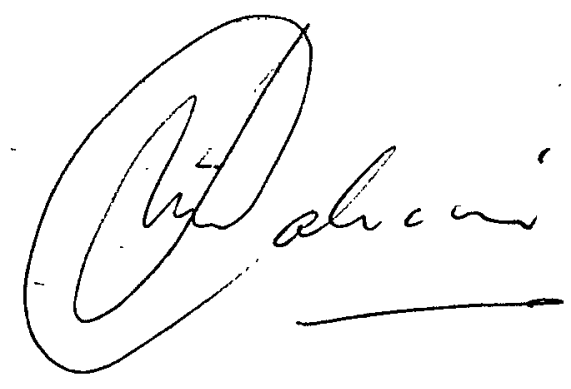

\section{Access to, and copying of, thesis}

The thesis copy lodged in the University Library shall be made available by the University for consultation but, for a period of two years after the thesis is lodged, it shall not be made available for loan or photocopying without the written consent of the author and in accordance with the laws of copyright.

After a thesis has been examined, the following authority will apply. Please complete your request, and sign below.

(i) I agree/do gree that the thesis may be made avala

(ii) I agree/ that the thesis may be made available for photocopying.

(iii) I note that my consent is required only to cover the two-year period following approval of my thesis for the award of my degree. After this, access to the Library copy will be subject only to any general restrictions laid down in Library regulations.

Signed:

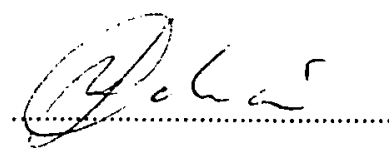<smiles>CC(C)(C)C(C)(C)C(C)(C)C(C)(C)C(C)(C)C</smiles>

Lodged in Morris Miller Central Library: $12 . . .1 . . . \ldots / 198 \ldots .9$ from which date the two years embargo will apply. 
I certify that this thesis contains no material which has been accepted for the award of any other higher degree or graduate diploma in any university, and that to the best of my knowledge and belief the thesis contains no copy or paraphrase of material previously published or written by another person, except where due reference is made in the text of the thesis. 


\begin{abstract}
Many aspects of human respiration are known to change from wakefulness to NREM sleep, including the level of ventilation, and the responsiveness to chemical respiratory stimuli. The differences in ventilation between wakefulness and sleep are due to a number of factors, one of which has been hypothesised to be the cessation of a wakefulness drive to respiration. Such an hypothesis would link the stimulation of respiration to the activity within the ascending reticular activating system, activity which varies greatly during the period of sleep onset, and which is responsible for the changes in activity seen at the cerebral cortex. The hypothesis allows the prediction that cessation of the wakefulness drive would occur rapidly and with a consistent relationship to EEG changes during the sleep onset period.
\end{abstract}

The present literature lacks an accurate description of the relationship between changes in ventilation and alterations in cortical arousal as measured by the EEG. This thesis reports three experiments designed to investigate ventilation during sleep onset and to provide such a description, in both males and females. An assessment is also made of the extent to which respiratory data collected over the sleep onset period is influenced by the measurement devices used.

In the first study, sleep onsets were studied in five young male adults in a series of single subject designs in which sleep onsets were replications. The results indicated that during sleep onset, the commencement of continuous theta activity in the EEG was associated with a substantial, rapid and highly predictable reduction in ventilation. The change in ventilation was typically due to a reduction in tidal volume, and was in part secondary to a reduction in metabolic rate. It was speculated that the non-metabolic component may reflect the loss of a wakefulness drive to respiration, though other interpretations were not eliminated. 
Sex differences exist in the incidence of sleep related respiratory dysfunction. In addition, there is uncertainty as to whether females show sleep related decreases in the responsiveness to chemical stimuli. This uncertainty has often been explained in terms of different subjects being in different cycles of the menstrual cycle when investigated. These data led to doubt as to whether females possess a wakefulness drive to respiration and, if so, whether it is affected by progesterone levels.

The second experiment sought to investigate ventilation during sleep onset in normal young females, during both phases of the menstrual cycle. Sleep onsets were studied in five subjects in a series of single subject designs. The results obtained supported those of the male subjects. That is, decreases in ventilation were associated with the onset of theta activity in the EEG. Further, no menstrual phase differences existed in the magnitude of the ventilation decrease or in its timing relative to EEG variations. Thus, females demonstrated the same pattern of ventilation change as males, indicating that they also possess a wakefulness drive.

In the first two experiments ventilation was monitored with a mask and breathing valve attached to a pneumotachograph and flow meter. There is evidence that during wakefulness, the increased dead space and respiratory load associated with such equipment substantially elevates the level of ventilation. The existence of such an effect while awake, but not during sleep, would also explain the above results. The third experiment was designed to determine whether the decrease in ventilation, associated with the onset of theta activity, could also be obtained without the mask and associated apparatus.

Two young male subjects were investigated in three conditions. First, wearing a mask as per the previous experiments as well as abdominal and thoracic strain gauges. Second, wearing a nasal catheter with strain gauges, and third, wearing the strain gauges 
only. Once again the subjects were treated as single case designs with sleep onsets within each condition as replications. Data from the first condition was used to construct regression equations to enable the estimation of tidal volume from the strain gauges.

Each subject required different regression equations for wakefulnes and sleep to accurately estimate ventilation from the strain gauge data. Different regression equations were also required for each unique set of strain gauges used. The data indicated that the practice in earlier studies, of estimating ventilation during sleep, using regression equations based on short calibration sessions held during wakefulness, has probably involved the introduction of error.

Both subjects displayed decreases in ventilation in all three conditions, associated with the onset of theta activity in the EEG. The elevation of the ventilation level associated with the mask did not vary significantly between wakefulness and sleep. Thus the data from the first two experiments was not produced by the use of specific respiratory apparatus, and is thus consistent with the wakefulness drive hypothesis. 


\section{ACKNOWLEDGEMENTS}

I am extremely thankful for the work of my supervisor, John Trinder. His inspiration, friendship and assistance with the data analysis and writing of the thesis are all greatly appreciated. Thanks must also go to Iain Montgomery, who has provided much needed practical and moral support as a second supervisor. Special acknowledgement is also due to Geoff Fraser, who assisted with the scoring of sleep records and suffered more or less silently through three years of sharing an office with me.

Only the willing and capable support of the technical staff of the Psychology Department enabled the equipment demands of data collection to be met. I wish to thank John Wanless, Brian Rieusett, Steve Bradford, Richard Rogers and Bob Reid for designing, building and maintaining the equipment used and Peter Tattam for writing and constantly updating the computer software.

I would like to thank the following people who assisted with the collection of blood for analysis in the second experiment: Sr. Fiona Urquhart; Dr. Ian Taylor; Sr. Jean Brown; and the staff of the University Student Health Clinic. Thanks are also due to the staff of the Department of Clinical Chemistry, Royal Hobart Hospital, who conducted the analysis of the samples.

The studies of this thesis were very demanding on the participating subjects. Without their understanding and willing participation the studies would have been much more difficult to complete. I am very grateful to all of them.

Finally, and most importantly, I would like to express my deepest gratitude to my parents, and to my wife Carol. Without the slightest complaint, they have tolerated the erratic hours and fragile temperament associated with my conducting sleep experiments over the last three years. In addition, wherever possible they have unselfishly provided much needed practical assistance. It was their support and love which really made this thesis possible. 


\section{ACKNOWLEDGEMENTS}

I am extremely thankful for the work of my supervisor, John Trinder. His inspiration, friendship and assistance with the data analysis and writing of the thesis are all greatly appreciated. Thanks must also go to Iain Montgomery, who has provided much needed practical and moral support as a second supervisor. Special acknowledgement is also due to Geoff Fraser, who assisted with the scoring of sleep records and suffered more or less silently through three years of sharing an office with me.

Only the willing and capable support of the technical staff of the Psychology Department enabled the equipment demands of data collection to be met. I wish to thank John Wanless, Brian Rieusett, Steve Bradford, Richard Rogers and Bob Reid for designing, building and maintaining the equipment used and Peter Tattam for writing and constantly updating the computer software.

I would like to thank the following people who assisted with the collection of blood for analysis in the second experiment: Sr. Fiona Urquhart; Dr. Ian Taylor; Sr. Jean Brown; and the staff of the University Student Health Clinic. Thanks are also due to the staff of the Department of Clinical Chemistry, Royal Hobart Hospital, who conducted the analysis of the samples.

The studies of this thesis were very demanding on the participating subjects. Without their understanding and willing participation the studies would have been much more difficult to complete. I am very grateful to all of them.

Finally, and most importantly, I would like to express my deepest gratitude to my parents, and to my wife Carol. Without the slightest complaint, they have tolerated the erratic hours and fragile temperament associated with my conducting sleep experiments over the last three years. In addition, wherever possible they have unselfishly provided much needed practical assistance. It was their support and love which really made this thesis possible. 


\section{TABLE OF CONTENTS}

\section{Page}

ABSTRACT iii

ACKNOWLEDGEMENTS vi

GLOSSARY xi

LIST OF TABLES .

LIST OF FIGURES Xiv

Chapter One: Introduction. 1

Chapter Two: Respiration during NREM sleep. 5

2.1 The Level of Ventilation. 6

2.2 Chemical Control Mechanisms. 8

2.3 Metabolic Rate. 10

2.4 Physical Properties. $\quad 11$

2.5 Animal studies. 12

2.6 Summary. 14

Chapter Three: The Nature of Respiratory Control and the Evidence for a Wakefulness Drive to Respiration. 16

3.1 Respiratory Control Mechanisms. . 17

3.2 Reticular Influences on Awake - Sleep Transitions. 19

3.3 Evidence for a Wakefulness Drive to Respiration. 20 
3.3.1 The Level of Ventilation in NREM Sleep. $\quad 20$

3.3.2 Post-hyperventilation Apnea. 21

3.3.3 Clinical Evidence for a Wakefulness Drive. 22

3.3.4 Changes in Brain Stem Influences on 23 Respiration.

3.3.5 Higher Brain Centre Influence on Respiratory 25 Control.

3.4 The Relationship between EEG and Measured Respiratory Changes.

3.5 Summary.

Chapter Four: Experiment One: Ventilation During Sleep Onset in Male Subjects.

4.1 Introduction. $\quad 31$

4.2 Method. 31

4.2.1 Subjects and Design. 31

4.2.2 Equipment. 32

4.2.3 Variables. 33

4.2.4 Data Analysis. ‥ 34

4.3 Results. $\quad 36$

4.4 Discussion. $\quad 52$

Chapter Five: The Evidence for Sex Differences in Respiration. 53

5.1 Ventilation During NREM Sleep in Females. 54

5.2 Sex Differences in Chemical Control Mechanisms. 55

5.2.1 Awake Differences. . 55

5.2.2 Sleep Related Changes. 56 
5.3 Evidence for Sex Differences in Sleep Related

Respiratory Dysfunction.

5.4 Evidence for Progesterone as a Respiratory Stimulant. 59

5.4.1 Luteal - Follicular Comparisons. 60

5.4.2 Respiration During Pregnancy. 61

5.4.3 Progesterone as Therapy for Respiratory 62

Dysfunction.

5.4.4 Effects in Normal Males and Experimental

Animals.

5.4.5 Mechanism of Action. 63

5.5 The Role of Testosterone in Sex Differences. 64

5.6 Summary. $\quad \dot{6} \quad \dot{65}$

Chapter Six: Experiment Two: Ventilation During Sleep Onset in Normal Females. $\quad 66$

6.1 Introduction. 67

6.2 Method. 67

6.2.1 Subjects and Design. 67

6.2.2 Equipment. 69

6.2.3 Variables. 69

$\begin{array}{lll}\text { 6.2.4 Data Analysis. } & 70\end{array}$

6.3 Results. 70

6.3.1 Progesterone Assay. 70

6.3.2 General Findings. 71

6.3.3 Luteal - Follicular Comparison. 84

6.4 Discussion. 92 
Chapter Seven: Methodological Considerations. 95

7.1 Sensory stimulation. 96

7.2 Upper-airway Resistance. 98

7.3 Apparatus effects. 98

7.4 Implications for Awake - Asleep comparisons. 101

Chapter Eight: Experiment Three: Equipment Artifact and Ventilation During 103 Sleep Onset.

8.1 Introduction. 104

8.2 Method. 104

8.2.1 Subjects and Design. 104

8.2.2 Equipment. 105

8.2.3 Variables. 106

$\begin{array}{lll}8.3 & \text { Results } & 107\end{array}$

8.3.1 Multiple Regression Analysis. $\quad 107$

8.3.2 Ventilation During Sleep Onset. 111

$\begin{array}{lll}8.4 & \text { Discussion. } & 122\end{array}$

$\begin{array}{ll}\text { Chapter Nine: Discussion and Conclusions. } & 124\end{array}$

9.1 Male Subjects. 126

9.2 Female Subjects. 126

9.3 The Effect of a Mask on Ventilation. $\quad 127$

9.4 Summary. $\quad 129$

9.5 Implications for Future Research. 130

References. 129

$\begin{array}{ll}\text { APPENDICES } & 151\end{array}$ 


\section{GLOSSARY}

CD Respiratory cycle duration.

EEG Electroencephalograph.

EMG Electromyograph

EOG Electro-oculograph.REM

FetCO 2 Estimate of end-tidal carbon dioxide based on expired air samples.

$\mathrm{FetO}_{2} \quad$ Estimate of end-tidal oxygen based on expired air samples.

$\mathrm{P}_{\mathrm{A}} \mathrm{CO}_{2} \quad$ The partial pressure of carbon dioxide at the alveoli.

REM Rapid Eye Movement sleep̀.

$\mathrm{VCO}_{2} \quad$ Minute carbon dioxide production.

VE Minute ventilation of expired air.

VE(Extrap) Minute ventilation of expired air extrapolated from single breath $\mathrm{Vt}$ and $\mathrm{CD}$ values.

VE(Extrap)* Minute ventilation of expired air extrapolated from single breath $\mathrm{Vt}(\mathrm{Regr})$ and $\mathrm{CD}$ values.

VE(Regr) Minute ventilation of expired air estimated from regression equations using abdominal and thoracic strain guage measurements.

$\mathrm{VE} / \mathrm{VO}_{2}$ The ventilation equivalent calculated by dividing VE by $\mathrm{VO}_{2}$.

$\mathrm{VO}_{2} \quad$ Minute oxygen consumption.

$\mathrm{V} / \mathrm{PCO}_{2}$ Ventilation related to changes in the partial pressure of carbon dioxide.

Vt Tidal volume.

$\mathrm{Vt}(\mathrm{Regr}) \quad$ Tidal volume estimated from regression equations using abdominal and thoracic strain guage measurements. 


\section{LIST OF TABLES}

Page

TABLE 4.1 Respiration during sleep onset: Minute values for male subjects.

TABLE 4.2 The relationship during sleep onset between significant decreases in ventilation and EEG activity in male subjects.

TABLE 4.3 Average correlations between EEG and respiratory variables during particular phases of sleep onset in male subjects.

TABLE 4.4 Breath by breath changes in respiratory variables before and after the transition from EEG alpha/beta to theta activity in male subjects.

TABLE 4.5 Breath by breath changes in respiratory variables in alpha - theta - alpha transitions for male subjects.

TABLE 6.1 Respiration during sleep onset: Minute values for female subjects.

TABLE 6.2 The relationship during sleep onset between significant decreases in ventilation and EEG activity in female subjects. $\quad 76$

TABLE 6.3 Average correlations between EEG and respiratory variables during particular phases of sleep onset in female subjects.

TABLE 6.4 Breath by breath changes in respiratory variables before and after the transition from EEG alpha/beta to theta activity in female subjects.

TABLE 6.5

Breath by breath changes in respiratory variables in alpha - theta - alpha transitions for female subjects. 
TABLE 6.6 Respiration during sleep onset: Minute values for

follicular and luteal menstrual phases.

TABLE 6.7 Summary of the analysis of the timing of ventilatory decreases comparing follicular with luteal phase.

TABLE 6.8 Summary of the analysis of the correlation coefficients between ventilation and EEG state comparing follicular with luteal phase.

TABLE 6.9 Breath by breath changes in respiratory variables before and after the transition from EEG alpha/beta to theta activity in follicular and luteal phases.

TABLE 8.1 Regression equations relating abdominal and thoracic movement to tidal volumes.

TABLE 8.2 Respiration during sleep onset : Minute values for the MASK, CATH and GAUGE conditions.

TABLE 8.3 Breath by breath changes in respiratory variables before and after the transition from EEG alpha/beta to theta activity in the MASK, CATH and GAUGE conditions. 116

TABLE 8.4 Summary ANOVA tables for the analysis of equipment condition. 


\section{LIST OF FIGURES}

FIGURE 4.1 Minute ventilation for subject D.L. during the final 10 minutes of wake, the transitional period of mixed wake/stage 1 sleep, and the first 10 minutes of stage 2 sleep.

FIGURE 4.2 Minute ventilation extrapolated from individual breaths for subject M.W. The values for the final 5 breaths before cessation of alpha in the EEG, and the following 35 breaths are shown.

FIGURE 4.3 Tidal volume for subject M.W. The values for the final 5 breaths before cessation of alpha in the EEG, and the following 35 breaths are shown.

FIGURE 6.1 Minute ventilation for subject G.O. during the final 5 minutes of wake, the transition period of mixed wake/stage 1 sleep, and the first 10 minutes of stage 2 sleep.

FIGURE 6.2 Minute ventilation extrapolated from individual breaths for subject J.S. The values for the final 5 breaths before cessation of alpha in the EEG, and the following 35 breaths are shown. 
FIGURE 6.3 The ventilation equivalent for subject J.B. for the luteal and follicular menstrual phases. Data is presented from the last 5 minutes of wake, the transitional period of mixed wake / stage 1 sleep and the first 10 minutes of stage 2 sleep.

FIGURE 8.1 Minute values of end tidal $\mathrm{CO}_{2}$ for subject A.C. during the last five 5 minutes of wake, the transitional period of mixed wake / stage 1 sleep and the first 10 minutes of stage 2 sleep. Data is presented from the MASK and CATH conditions.

FIGURE 8.2 Minute ventilation extrapolated from individual breaths for subject A.C. The values of the final 5 breaths before cessation of alpha in the EEG and the following t 35 breaths are shown. Data is presented from the MASK, CATH, and GAUGE conditions.

FIGURE 8.3 End tidal Oxygen for subject D.L. The values of the final 5 breaths before cessation of alpha in the EEG and the following 35 breaths are shown. Data is presented from the CATH and MASK conditions. 
CHAPTER ONE

INTRODUCTION 


\section{CHAPTER ONE}

\section{INTRODUCTION}

Reports published over the last one hundred years suggest that breathing differs between wakefulness and sleep (Douglas, 1984). The early studies using behavioural identification of sleep, and more recent investigations using electrophysiological methods, have typically shown a lower level of ventilation in sleep, particularly non rapid eye movement (NREM) sleep. In addition, recent studies have shown variations in the chemical control and physical mechanics of respiration during sleep.

This thesis was concerned with the change which occurs in ventilation during sleep onset. Informal observations reported in the literature have suggested that this change occurs systematically at the point of sleep onset. The aim of this thesis was to test the validity of these observations. However, as has been reported by a number of authors, the sleep onset period is difficult to analyse (Hudgel \& Devadatta, 1984; Hudgel, Martin, Johnson \& Hill, 1984; Tabachnik, Muller, Bryan \& Levison, 1981; Yamashiro, Fukushima, Okudaira, Suzuku \& Nishi, 1987). To a large extent, this difficulty stems from the fact that the transition from wakefulness to sleep is characterised by a succession of rapid oscillations in arousal level before stable sleep is achieved. In order to successfully describe ventilation during this period, it was necessary to develop both particular analysis techniques for resolving data and computer software which allowed a breath-by-breath analysis of ventilation during the transitional period.

The description of ventilatory change during sleep onset is pertinent to understanding the respiratory control mechanism responsible for the change. It has been suggested that the reduction in ventilation during sleep is a consequence of the cessation of a "wakefulness drive" to ventilation (Sullivan, 1980, p. 221). On the basis of this theory of respiratory control, it was predicted that the ventilation change during sleep onset would be rapid and systematically associated with changes in cortical arousal. 
Chapter two reviews the literature which has reported changes in respiration known to occur with NREM sleep in humans and other animal species. It does not include a review of changes in respiration associated with REM sleep. This review of respiratory changes during sleep emphasises NREM sleep because in normal adults, sleep begins with NREM sleep. The NREM stages 2, 3, and 4 are followed by REM sleep approximately 90 minutes later. The alteration of NREM and REM then continues throughout sleep in approximately 90 minute cycles.

There are a number of points of evidence for a wakefulness drive to respiration. These include: the differences in ventilation and chemical ventilatory drives between wakefulness and sleep; the occurrence of apnea following hyperventilation during sleep; the existence of respiratory diseases with state specific symptomatologies; and the fact that sleep-related changes occur in neural activity in brain stem arousal and respiratory centres. This evidence is reviewed in Chapter three. Chapter four describes an experiment conducted on normal male subjects which investigated ventilation changes associated with sleep onset.

A male predominance in disordered breathing during sleep has been reported (Block, Boysen, Wynne \& Hunt, 1979), implying a sex difference in respiration and perhaps in respiratory control. The greater levels of circulating progesterone in females during the luteal phase could contribute to these differences. Chapter five reviews the evidence for sex differences in respiration during wakefulness and sleep, and the evidence for progesterone acting as a respiratory stimulant. The possible existence of sex differences in respiratory control suggests that the change in respiration at sleep onset may also differ between males and females. Chapter six presents a study which investigated respiration during sleep onset in female subjects. 
When investigating human respiration, one has to compromise between the need to make direct measurements of physiology and the need to use a non-invasive methodology. It is known that the apparatus used in many experiments has systematic influences on respiration (Gilbert, Auchincloss, Brodsky \& Boden, 1972). If the effects of the equipment itself are specific to either wakefulness or sleep, comparisons of the two states are likely to produce erroneous results. Chapter seven reviews the literature concerning these influences. Chapter eight presents the results of an investigation into the effects of the apparatus used in the previous two studies of the present thesis. Finally, Chapter nine discusses the theoretical implications of the three studies of the thesis. 


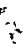

CHAPTER TWO

RESPIRATION DURING NREM SLEEP 


\section{CHAPTER TWO \\ RESPIRATION DURING NREM SLEEP}

\subsection{The level of ventilation}

There is a substantial literature which indicates that many aspects of respiratory function vary between wakefulness and NREM sleep. Minute ventilation has been consistently found to decrease with sleep (Birchfield, Sieker \& Heyman, 1959; Bulow, 1963; Bulow \& Ingvar, 1961; Douglas White, Pickett, Weil \& Zwillich, 1982a; Douglas, White, Weil, Pickett, Martin, Hudgel \& Zwillich, 1982b; Gleeson, Zwillich, Braier \& White, 1986; Gothe, Altose, Goldman \& Cherniack, 1981; Gothe, Cherniack \& Williams, 1986; Hudgel et al., 1984; Lopes, Tabachnik, Muller \& Levison, 1983; Lugaresi, Coccagna, Cirignotta, Farneti, Gallasi, Di Donato \& Verucchi, 1978; Magnussen, 1944; Reed \& Kellogg, 1958; 1960a; 1960b; Rist, Daubenspeck \& McGovern, 1986; Robin, Whaley, Crump \& Travis, 1958; Skatrud \& Berssenbrugge, 1983; Skatrud \& Dempsey, 1983; Tabachnik et al., 1981; White, 1986; White, Douglas, Pickett, Weil \& Zwillich, 1982; White, Weil \& Zwillich, 1985b; Yamashiro et al., 1987). In addition, Gothe, Goldman, Cherniack \& Mantey (1982), reported a non-significant decrease.

In many previous reviews there has been no differentiation between results which met, and those which failed to meet, tests of statistical significance. For example, the Gothe et al. (1982) study above, is often cited as providing evidence that ventilation decreases with sleep. The clear separation of significant and non-significant results in this thesis enables the presentation of all the available and relevant evidence in a more accurate manner. This approach is adopted throughout the thesis whenever citing from a number of studies in support of a particular argument. 
Shore, Millman, Silage, Chung \& Pack (1985), in a study comparing young and elderly subjects, reported a significant decrease in ventilation among the young group, with only a slight fall in the elderly. However, a similar study by Krieger, Turlot, Mangin \& Kurtz (1983) reported significant reductions in the ventilation of both young and old subjects, with no notable age effect. Finally, Duron (1972) and Duron, Andrac \& Laval (1967), provided the only reports to contradict the general finding, as they found no change in ventilation from wakefulness to sleep.

The sleep-related minute ventilation decrease is often associated with a decrease in tidal volume (Birchfield et al., 1959; Bulow, 1963; Bulow \& Ingvar, 1961; Douglas et al., 1982a; Gothe et al., 1981; Gothe et al., 1986; Hudgel et al., 1984; Lopes et al., 1983; Magnussen, 1944; Skatrud \& Berssenbrugge, 1983; White, 1986; White et al., 1982; White et al., 1985b). Gothe et al. (1982) and Tabachnik et al. (1981) reported non-significant decreases. Shore et al. (1985) reported a sleep associated decrease in tidal volume among young subjects. Elderly subjects displayed an increase in tidal volume during stages one and two when compared with wakefulness, however slow wave sleep values were below those of wakefulness.

Reports of respiratory rate changes with NREM sleep have been more variable than those of tidal volume. In a number of studies, respiratory rate has been reported to decrease during sleep (Gothe et al., 1986; Khatri \& Freis, 1967; Lopes et al., 1983; Shore et al., 1985; Snyder, Hobson, Morrisson \& Goldfrank, 1964; Tabachnik et al., 1981; Yamashiro et al., 1987). However, increases have also been reported (Birchfield et al., 1959; Douglas et al., 1982a; Duron et al., 1967; Hudgel et al., 1984; Kumar, van Diest, Hofman, Visser, Poelstra \& Bakker, 1980). Finally, Gothe et al. (1981; 1982) reported a non-significant decrease with sleep, and White (1986) reported "little change". 


\subsection{Chemical Control Mechanisms}

Associated with the lower level of ventilation during sleep, are increases in the partial pressure of carbon dioxide at the alveoli and in arterial blood (Birchfield et al., 1959; Bulow, 1963; Bulow \& Ingvar, 1961; Coccagna \& Lugaresi, 1978; Douglas et al., 1982a; Douglas, White, Weil, Pickett \& Zwillich, 1982c; Gothe et al., 1981; Gothe et al., 1982; Gothe et al., 1986; Hedemark \& Kronenberg, 1982; Magnussen, 1944; Naifeh \& Kamiya, 1981; Naifeh, Kamiya \& Monroe-Sweet, 1982; Reed \& Kellogg, 1958; 1960a; 1960b; Rist et al., 1986; Robin et al., 1958; Shore et al., 1985; Townsend, Prinz \& Obrist, 1973; White, 1986; White et al., 1982). There is also a decrease in blood pH (Birchfield, 1959; Coccagna \& Lugaresi, 1978; Robin et al., 1958). These changes indicate an increased tolerance for carbon dioxide during sleep.

Another manifestation of increased tolerance for carbon dioxide is a sleep related decrease in the ventilatory response to increases in carbon dioxide (Bellville, Howland, Steed \& Houde, 1959; Berthon-Jones \& Sullivan, 1984; Birchfield et al., 1959; Bulow, 1963; Bulow \& Ingvar, 1961; Douglas et al., 1982c; Gothe et al., 1981; Gothe et al., 1986; Magnussen, 1944; Naifeh, Severinghaus \& Kamiya, 1987; Reed \& Kellogg, 1958; 1960b; Rist et al., 1986; Robin et al., 1958). The one exception to these findings was provided by Hedemark \& Kronenberg (1982), who found no difference between awake and NREM sleep hypercapnic responses.

These results indicate that there is a variation in the metabolic control of ventilation between the awake and NREM sleep states. The change in the hypercapnic ventilatory response usually involves a shift of the $\mathrm{V} / \mathrm{PCO}_{2}$ curve to the right, and a slight reduction in slope (Cherniack, 1981). While this finding is well established, there is also some evidence of sex differences (Berthon-Jones \& Sullivan, 1984; and Newsom-Davis, Loh, Nodal, Charnock, 1978), the implications of which are discussed in Chapter five.

Several studies have reported that NREM sleep is associated with a decrease in 
oxygen partial pressure, or oxyhaemoglobin saturation, in normal subjects (Birchfield et al., 1959; Coccagna \& Lugaresi, 1978; Douglas et al., 1982a; Gothe et al., 1986; Kopelman, Apps, Cope \& Empey, 1985; Reed \& Kellogg, 1958; Tabachnik et al., 1981).However, Robin et al. (1958), reported a non-significant decrease in oxygen partial pressure and no change in saturation. Peset (1984), found no change in saturation and Gothe et al. (1982) only a slight decrease.

The general opinion of reviewers is that hypoxic ventilatory responses are little altered by NREM sleep (Cherniack, 1981; Phillipson, 1978). This belief is supported by three recent studies which failed to find a state-related difference (Gothe et al., 1986; Hedemark \& Kronenberg, 1982; White et al., 1982). A dissenting view is that of Douglas (1984), who cited two studies which reported a sleep-related decrease in the response (Berthon-Jones \& Sullivan, 1982; Douglas et al., 1982b). The uncertainty of conclusions in the literature are exemplified by the study by Gothe et al. (1982), in which 17 young adults were investigated. Of these, ten demonstrated no effect, five a significant increase, and two a significant decrease in response during NREM sleep. As with the hypercapnic response, there is some evidence of a possible sex difference (White et al., 1982) which is discussed in Chapter five. 


\subsection{Metabolic Rate}

It has been demonstrated that metabolic rate decreases from wakefulness to NREM sleep. This has been the case both for measurements of oxygen consumption (Brebbia \& Altshuler, 1965; Duron et al., 1967; Haskell, Palca, Walker, Berger \& Heller, 1981; Kreider \& Iampietro, 1959; Magnussen, 1944; Robin et al., 1958; Webb \& Hiestand 1975; White et al., 1985b; Yamashiro et al., 1987), and carbon dioxide production (Bulow, 1963; Duron et al., 1967; Robin et al., 1958; Shapiro, Goll, Cohen \& Oswald, 1984; White et al., 1985b; Yamashiro et al., 1987).

The major function of ventilation is to provide the oxygen required for metabolism and to remove the carbon dioxide produced. It could be argued that the lowering of metabolic rate during NREM sleep is the major factor leading to the reported decrease in ventilation. This hypothesis is supported by studies demonstrating a strong correlation between ventilation and metabolic rate measurements (Phillipson, Bowes, Townsend, Duffin, Cooper, 1981; Phillipson, Duffin, Cooper, 1981; White et al., 1985b). However, the increased carbon dioxide partial pressure associated with sleep indicates that ventilation decreases by an amount greater than that required by a decrease in metabolic rate (Sullivan, 1980). Further, a metabolic rate explanation of the decrease in ventilation is insufficient to explain the observed variations in the chemical control mechanisms. The implications of these issues are discussed in Chapter three. 


\subsection{Physical Properties}

There are a number of changes to the mechanics of respiration which occur with NREM sleep. In wakeful, supine subjects, there is a greater abdominal (diaphragmatic) contribution to respiration than thoracic (intercostal muscles) (Goldman, 1982; Sharp, Goldberg, Druz \& Danon, 1975). Most researchers report that during NREM sleep this situation is reversed, there being relatively more thoracic breathing (Goldie \& Green, 1961; Gothe et al., 1981; Gothe et al., 1982; Mortola \& Anch, 1978; Naifeh \& Kamiya, 1981; Tabachnik et al., 1981; Timmons, Salamy, Kamiya \& Girton, 1972). At least one study failed to find a difference between awake and NREM sleep patterns (Tusiewicz, Moldofsky, Bryan \& Bryan, 1977). Naifeh \& Kamiya (1981) suggest that this finding is to be explained by the fact that Tusiewicz et al. (1977) did not measure lateral rib-cage expansion.

Tabachnik et al. (1981), in addition to measuring thoracic and abdominal movement, used intercostal and diaphragmatic electromyograms to measure respiratory muscle activity. Their results indicated that the increased thoracic contribution to respiration during sleep is the result of "active expansion of the rib cage by enhanced intercostal muscle activity" (p. 560). The increase in muscle activity, without increase in tidal volume, indicated an increase in respiratory work-load that was possibly due to an increase in upper airway resistance. Although a sleep related decrease in the functional residual capacity might also be involved (Hudgel \& Devadatta, 1984).

Increases in airway resistance have been found to occur with NREM sleep, either measured directly (Hudgel \& Devadatta 1984; Lopes et al., 1983), or inferred from changes in occlusion pressure and inspiratory flow rate (White, 1986). Lopes et al. (1983) reported an increase in intercostal (but not diaphragmatic) EMG in addition to the increase in airway resistance. They comment that by increasing rib cage expansion it is possible to increase trans-diaphragmatic pressure without altering diaphragmatic EMG 
activity. Lopes et al. (1983) interpreted these changes as indicating an increase in the efficiency of diaphragmatic contraction. Thus the abdominal to thoracic shift reported to occur with sleep could be viewed as an attempt to increase the efficiency of respiratory mechanics in response to an increase in respiratory load produced by the narrowing of the upper airway.

Unlike the above studies, Rist et al. (1986) attempted to adjust the awake and NREM-sleep EMG measures to correct for the different carbon dioxide partial pressures in each state. Following this adjustment, they reported significant decreases in intercostal and diaphragmatic EMG from wakefulness to NREM-sleep. They also noted, however, that for a given level of diaphragmatic and intercostal EMG activity, the ventilation produced during wakefulness was greater than that during sleep. They interpreted these results as evidence for both a decrease in the central respiratory drive and a reduced effectiveness of respiratory muscles during sleep. Taking all the results into consideration, they concluded that reduced mechanical efficiency accounts for only a portion of the total reduction in ventilation.

\subsection{Animal Studies}

All the studies cited thus far have used human subjects. A brief review of the literature involving animal studies reveals interesting similarities to, and differences from, the human studies. Most of the animal studies comparing the awake and synchronised (NREM) sleep states have concentrated on cats and dogs. However, Coote (1982) reviewed studies covering a variety of species. Coote's conclusion was that all animals demonstrated a reduction in respiratory rate with entry into synchronised (NREM) sleep from quiet wakefulness. This conclusion is supported by studies using dogs (Phillipson, Murphy \& Kozar, 1976; Phillipson, Sullivan, Read, Murphy \& Kozar, 1978; Sullivan, Kozar, Murphy \& Phillipson, 1978), cats (Duron \& Marlot, 1980; Orem, Netick \& Dement, 1977a; 1977b; Remmers, Bartlett \& Putnam, 1976) and rats (Pappenheimer, 1977). 
In animals, tidal volume has been found to increase with NREM sleep; this is the opposite of what typically happens in humans (Orem et al., 1977a; 1977b; Phillipson et al., 1976; Remmers et al., 1976). The reduction in minute ventilation can thus occur only because of a decrease in respiratory rate (Orem et al., 1977a; 1977b; Pappenheimer, 1977). Non-significant decreases in respiratory rate were reported by Phillipson et al. (1976) and Sullivan et al. (1978).

There are reports, using animals, of sleep related increases in carbon dioxide pressure (Guazzi \& Freis, 1969; Phillipson et al., 1976), and decreases in blood pH and oxygen pressure (Guazzi \& Freis, 1969). The hypercapnic ventilatory response has been found to decrease with sleep (Phillipson, 1977; Phillipson et al., 1976; Santiago, Shina \& Edelman, 1979). The hypoxic ventilatory response is reported as having shown no change (Phillipson, 1977; Phillipson et al., 1978) or to increase (Pappenheimer, 1977). Metabolic rate has been reported to decrease with sleep (Mayevsky \& Samuel, 1975).

Measurement of muscle activity in the major respiratory groups and the airways is easier and more direct in animals than in humans. A number of studies have reported decreased tone in upper airway musculature during NREM sleep (Megirian \& Sherrey, 1980; Megirian, Cespuglio \& Jouvet, 1978; Orem \& Lydic, 1978; Orem et al., 1977b; Sherrey \& Megirian, 1977; Sherrey \& Megirian, 1980). Intercostal muscle tone has been shown to decrease (Parmeggiani \& Sabattini, 1972) or to remain the same (Duron \& Marlot, 1980). Diaphragmatic activity seems little altered by NREM sleep (Remmers et al., 1976; Duron \& Marlot, 1980).

Data relevant to the role of the upper airways in sleep related respiratory changes is found in three studies in which the dogs used were prepared surgically with a permanent tracheostomy (Phillipson et al., 1976; Phillipson et al., 1978; Sullivan et al., 1978). In these studies, the dogs displayed the typical changes associated with sleep as described 
above, despite a lack of variation in upper airway patency. These results add vital information to our understanding of the decrease in ventilation with NREM sleep. They clearly indicate that such a change can, and does occur in the absence of the increased respiratory load associated with upper airway narrowing during sleep. Thus there is data from animals as well as humans (Rist et al., 1986) to indicate that respiratory changes with sleep require more than changes in the efficiency of the mechanics of breathing. That is, they may be due also to a decrease in central respiratory drive.

\subsection{Summary}

A reduced level of ventilation during NREM sleep is common in humans and other animal species. In man this tends to be mediated by a decrease in tidal volume with variable effects on respiratory rate. In cats and dogs the opposite is the case, with respiratory rate showing consistent decreases and tidal volume showing little change, or even an increase. The carbon dioxide control mechanism varies in man and animals, NREM sleep being associated with an increase in carbon dioxide pressure and a decrease in the hypercapnic ventilatory response. The effects of NREM sleep on the oxygen control system are less clear, as not all investigations report decreases in the hypoxic ventilatory response. Metabolic rate has been found to decrease with sleep whether measured by oxygen consumption or carbon dioxide production.

Human subjects experience consistent changes in the contribution of the thorax and diaphragm to respiration. Sleep is associated with a greater thoracic expansion (when subjects are supine), and a decrease in upper airway muscle tone. The reduction in metabolic rate and increase in airway resistance are possibly sufficient to explain the other changes between wakefulness and sleep. 
The next chapter addresses the possibility that, in addition to a decreased metabolic rate and increased airway resistance, some central change in respiratory control is required to produce the changes to ventilation associated with sleep. 
CHAPTER THREE

THE NATURE OF RESPIRATORY CONTROL AND THE EVIDENCE FOR A WAKEFULNESS DRIVE TO RESPIRATION 


\section{CHAPTER THREE}

\section{THE NATURE OF RESPIRATORY CONTROL AND THE EVIDENCE FOR A WAKEFULNESS DRIVE TO RESPIRATION}

One cause of the sleep related variations in respiration is thought to be the cessation of a central drive to respiration associated with wakefulness. In this chapter, an outline of the mechanisms responsible for respiratory control and those involved in loss of wakefulness is presented. The direct and indirect evidence used in support of an hypothesised wakefulness drive to ventilation is then reviewed.

\subsection{Respiratory Control Mechanisms}

Berger, Mitchell \& Severinghaus $(1977 \mathrm{a}, \mathrm{b}, \mathrm{c})$ provide a three part review of the regulation of respiration. This review emphasises both the diversity of the sources of afferent information related to respiratory control, and the necessity of central integration of this information for the generation of appropriate motor commands. The first source of afferent information is provided by the peripheral chemoreceptor mechanisms. These lie within the ascending aorta (aortic bodies) and at the bifurcation of the common carotid arteries (carotid bodies). Both are stimulated by decreased partial pressures of oxygen and increased partial pressures of carbon dioxide. However, they vary in their response to decreased $\mathrm{pH}$, the carotid sensor being stimulated and the aortic sensors unaffected or depressed.

The fact that ventilatory responses to increases in carbon dioxide are maintained following denervation of peripheral chemoreceptors indicates that chemoreception is also performed by central mechanisms. Berger et al. (1977a) also state that " the transient response to step changes in alveolar carbon dioxide has a slow component with a time constant of about sixty seconds" (p. 94). This has led to the view that central chemoreception acts via a mechanism that does not permit "instant equilibration" with arterial blood. It is likely that the mechanism is a structure located on the ventro-lateral 
surface of the medulla that is sensitive to the $\mathrm{pH}$ of cerebro spinal fluid (Comroe, 1974; Berger et al., 1977a).

There are a number of mechanisms besides chemoreceptors for oxygen and carbon dioxide, that are capable of influencing respiration. Within the upper airways there are chemical and mechanical irritant sensors (Berger et al., 1977a). Those within the nasal airways have afferent pathways in the trigeminal and olfactory nerves. Those in the epipharynx transmit information via the pharyngeal branch of the glossopharyngeal nerve. Finally, those within the larynx transmit via the internal branch of the superior laryngeal nerve. The lower airways and lungs also contain a variety of receptors. Information from pulmonary stretch receptors located in the airway smooth muscle is transmitted via large myelinated vagal fibres and is thought to be important in determining the rate and depth of breathing. Two other types of lung receptors, irritant receptors and juxtapulmonary capillary receptors, also transmit information via vagal fibres.

Within the brain stem are three separate areas responsible for respiratory control. The most rostral of these is the pneumotaxic centre located bilaterally in the rostral pons. Stimulation of this area causes activation of the phrenic nerve and the switching of respiratory phase. The isolation of the pneumotaxic centre and vagal afferents from more caudal structures results in prolonged apneusis which points to an apneustic centre located in the region of the caudal pons or rostral medulla. Lastly, the medulla in continuation with the spinal cord, but isolated from more rostral influence, is able to produce rhythmical respiration (gasping). However, Berger et al. (1977b) point out that " ... when portions of the medulla are destroyed all automatic respiration ceases" (p. 140). Within the medulla there are two aggregations of nuclei that affect respiration. The dorsal respiratory group is associated with the ventrolateral portion of the nucleus of the solitary tract, and the ventral respiratory group with the nucleus ambiguus and nucleus retroambiguus. 
The dorsal respiratory group consists predominantly of inspiratory related neurons with projections to the contralateral spinal cord, approximately two-thirds of them monosynaptically exciting contralateral phrenic motoneurons. The fact that the nucleus of the solitary tract is the primary projection site of visceral afferents within the glossopharyngeal and vagus nerves implicates the dorsal respiratory group in the integration of afferent inputs into a respiratory motor response. This co-ordination / integration role is also supported by investigations indicating its unidirectional influence on the ventral respiratory group, which contains both inspiratory and expiratory neurons. The inspiratory neurons are concentrated in the rostral portion of the nucleus retroambiguus with the expiratory neurons being located more caudally. Approximately ninety percent of the neurons within the nucleus retroambiguus project to the contralateral spinal cord between the first thoracic and third lumbar segments, and influence intercostal and abdominal motoneurons.

The above description of the structures involved in the regulation of respiration provides a general overview. Of importance to the present thesis is the fact that a variety of afferent information is integrated in the brain stem and results in an organised patterns of respiratory motoneuron activity. The possible influence of reticular formation activity on these central "integrators" is discussed in section 3.3.

\subsection{Reticular Influences on Awake - Sleep Transitions}

The brain stem, in addition to containing structures involved in respiratory control, is central to the control of the cyclic fluctuations in arousal which lead to sleeping and waking behaviour. Relatively large fluctuations of the activity within the ascending reticular system are associated with the transition from sleeping to waking and vice versa (Schmidt, 1978). Further, brain stem reticular neurons, such as the raphe nuclei, are involved in wake-sleep transitions by virtue of their influence on levels of serotonin and other brain monoamines. (Schmidt, 1978). 
Remmers (1981) has argued that the reticular activating system maintains wakefulness by three mechanisms: a direct activation of cortical cells via reticulo-fugal projections; an activation of the major relay (lateral) nulcei of the thalamus, facilitating the passage of information through the thalamus to the cortex; and an inhibition of the medial thalamic nulcei, which exert an inhibitory influence on the lateral thalamic nuclei. He states that "the onset and progression of quiet sleep appears to involve reversal of the three neuronal processes by which the reticular activating system maintains wakefulness" (p. 116). Phillipson (1978) suggests that the onset of NREM sleep depends on the withdrawal of ascending reticular discharges and may depend also on the inhibition of the arousal system by basal forebrain mechanisms. This view is supported by Schmidt (1978), who claims that "even a chronically isolated brain lacking a reticular formation exhibits a sleeping-waking rhythm" (p. 288). Nonetheless the acquisition of sleep is typically associated with a decreases in ascending reticular activating system activity.

\subsection{Evidence for a Wakefulness Drive to Respiration}

"Wakefulness and facilitation of breathing are inextricable because of their common neural origins" (Orem, 1984, p. 95). Thus it can be hypothesised that changes in respiration associated with NREM sleep relate to the decrease of generalised wakefulness, or alertness, and thus imply a respiratory excitation by the reticular activating system (Hugelin and Cohen, 1963). The evidence in support of this wakefulness drive hypothesis is presented in the remainder of this chapter.

\subsubsection{The Level of Ventilation in NREM Sleep}

The studies reviewed in chapter two clearly indicate that NREM sleep is associated with a decreased level of ventilation. Further, the decrease is greater than that required by a sleep-related decrease in metabolic rate. This is demonstrated by the associated 
increase in carbon dioxide partial pressure.

Phillipson (1978) commented that a decrease in ventilation of one litre per minute and an increase in arterial $\mathrm{PCO}_{2}$ of five $\mathrm{mm}$. $\mathrm{Hg}$. can be completely accounted for by two factors, namely a small decrease in the metabolic production of carbon dioxide and a variation of the "homeostatic set-point" to a reduced alveolar ventilation, and an increased arterial $\mathrm{PCO}_{2}$. Evidence indicating an increased carbon dioxide "set point" is provided by the decreased ventilatory responsiveness to hypercapnia associated with NREM sleep. Phillipson hypothesises that the "change in 'set-point' is the direct result of the loss of the stimulating influence of wakefulness on breathing" (p. 920). This change in the carbon dioxide set-point is consistent with the wakefulness drive hypothesis, and cannot be explained by other postulated causes of the sleep related ventilation decrease, such as, increased airway resistance or decreased metabolic rate.

\subsubsection{Post-hyperventilation Apnea}

Additional evidence is provided for a wakefulness drive by studies which have investigated the effects of the artificial manipulation of ventilation during wakefulness and sleep. Douglas (1984) commented that reducing the end-tidal carbon dioxide level during NREM sleep to that found in wakefulness produces apnea in normal subjects. This conclusion summarises evidence from hyperventilation studies in humans and animals. For instance, Fink (1961) reported a complete absence of apnea following over-ventilation during wakefulness, despite a reduction of the end tidal carbon dioxide partial pressure to below $15 \mathrm{~mm}$. $\mathrm{Hg}$. in some instances. He comments that the absence of over-ventilatory apnea in conscious subjects, contrasts with the invariable onset of apnea in patients over-ventilated during general anesthesia, and can be explained in terms of a cerebral drive to ventilation associated with wakefulness.

A lack of apnea while awake was also reported by Tawadrous \& Eldridge (1974). 
However, these authors did not ascribe this result to the effects of wakefulness itself. They saw it as the result of active neural generation provided by the over-ventilation. This notion is supported by studies reporting apnea following passive hyperventilation in awake dogs (Mitchell, Bainton \& Edelist, 1966) and awake humans (Bainton \& Mitchell, 1966). Similarly Eldridge (1973), reported apnea after passively induced hyperventilation but not after active production in cats (where the active hyperventilation was produced by stimulation of the carotid sinus nerve).

There are however studies which support the notion that rhythmic ventilation during wakefulness is sustained by a wakefulness drive. Skatrud \& Dempsey (1983) and Skatrud \& Berssenbrugge (1983) both produced apnea in sleeping subjects, but failed to do so while the subjects were awake. In both experiments the hyperventilation was induced passively. Further, Fink (1961) did not find a significant difference between passive and active methods of hyperventilation in their ability to selectively produce apnea during sleep. In conclusion, it would appear that associated with wakefulness, there is a non-chemical stimulation of ventilation, and that this stimulation is sufficient to obviate the need for chemoreceptor mechanisms.

\subsubsection{Clinical Evidence for a Wakefulness Drive}

Clinical disorders which differentially effect respiration during wakefulness or sleep have been cited as evidence for a wakefulness drive. For example, Orr (1984) claimed that impressive evidence for the existence of a waking stimulus to breathe is available in case studies of humans with defective chemoreceptors, or damaged ventrolateral tracts who cease to breathe during sleep. The separation of the transmission of automatic and voluntary controls to respiratory muscles in different spinal tracts is often implicated in awake-sleep differences under clinical conditions. For example, Ondine's curse involves disruption of transmission only of automatic control, whereas respiratory apraxia effects only voluntary control (Cherniack, 1981). 
The presence of obstructive apnea during sleep, in patients who breathe normally while awake, can also be viewed as a reflection of the influence of wakefulness. St. John (1986), in a study using decerebrate, vagotomized, paralyzed and ventilated cats, found that motoneurons to upper airway muscles are more dependent upon medullary and pontine reticular mechanisms for their respiratory modulated activities than are motoneurons to the diaphragm. In some animals augmentations of reticular activity were necessary for respiratory modulated trigeminal activities to be manifested at all. Douglas (1984) has argued that this evidence, together with the changes in upper airway resistance during sleep, provide the necessary and sufficient elements to explain the presence of obstructive apnea at sleep onset.

Central apnea, while being much less common than the obstructive form, can also be viewed as partially due to the withdrawal of a wakefulness stimulus. White (1985) commented that normal sleepers respond less to both hypoxia and hypercapnia and have a reduced dependence on information from chemoreceptors in order to drive respiratory musculature. He concluded that with "further attenuation of ventilatory drive central apneas may occur" (p. 1210). Finally, Plum (1970) reviewed many clinical studies which indicated that medullary depression, or compression, can disproportionally damage the metabolic or automatic control of breathing. Typically, the result is that respiratory homeostasis can be maintained during wakefulness but not during sleep.

\subsubsection{Changes in Brain Stem Influences on Respiration}

It can be argued that the changes in ventilation associated with sleep should be reflected in the activity of brain stem neurons which have a relationship to respiration. For instance, Cohen (1981) identified the bulbopontine reticular formation as containing respiratory-related neurons. He cited experimental evidence that these neurons produce facilitatory effects on the primary respiratory rhythm generator. He also speculated that changes of respiratory-related neuron activity in the reticular formation "could be the basis of the fluctuations of respiratory neuron activity in the nucleus parabrachialis 
medialis in the pneumotaxic centre and in the medulla that are related to sleep-waking state" (p. 96).

Evidence that fewer medullary neurons fire in association with respiration during sleep was provided by Orem, Montplaisir \& Dement (1974). Working with cats, they identified twenty-two cells at the level of the facial nucleus, that displayed an alteration to their firing pattern between wakefulness and sleep. Seventeen reduced their discharge frequencies eventually ceased firing, and five reduced their intra-burst frequency while maintaining respiratory rhythmicity. While variation existed in the timing of the state related variations, the authors also reported an increased probability of altered activity with increasing depth of slow-wave sleep and with REM sleep. Orem \& Dement (1975) reported that the slower, more regular respiration in NREM sleep, was associated with long and regular discharge bursts from the ventrolateral medullary neurons. The conclusion of the authors, was that there was an apparent reduction in the number of medullary neurons showing respiratory related activity during sleep. In addition, the reduction began in NREM sleep (or even drowsiness) and progressed into REM (Orem \& Dement, 1975).

Orem (1984) reported data indicating that reticular neurons in the midbrain, rather than those in the medulla, were less active in NREM sleep than in wakefulness or REM sleep. He also observed that activity of midbrain intermittent respiratory neurons tended to be more likely in wakefulness than in NREM. On the basis of these observations he stated that "breathing is evidently supported by state specific processes in wakefulness and REM sleep but not in NREM sleep" (p. 95). He further suggested that the midbrain reticular formation facilitates breathing during wakefulness and that this influence is mediated through the forebrain. However, his suggestion must be qualified by the absence of evidence indicating direct projections from the midbrain reticular formation to the dorsal and ventral respiratory groups. 
An alternative approach to investigating brain stem involvement in a wakefulness drive has involved the manipulation of the quality and quantity of inputs allowed to reach brain stem structures. Sullivan et al. (1978) investigated dogs while awake and asleep, and with and without blockade of vagal afferent information. They reported that the combined loss of wakefulness, vagal stimulation, and peripheral chemoreception, led to the depression of ventilation (then under the sole control of central chemoreceptor mechanisms). The authors concluded that the results supported the concept of a tonic respiratory drive related to wakefulness, which, "mostly originates in the reticular activating system, has powerful effects on breathing and is clearly related to the sleeping - waking processes" (p. 16).

\subsubsection{Higher Brain Centre Influence on Respiratory Control}

An intrinsic feature of sleep is a change in the quantity and quality of cortical activity as compared with wakefulness. Early attempts to substantiate the hypothesis of a wakefulness drive postulated that such a drive would be mediated by the general level of activity in the central nervous system. Robin et al. (1958) hypothesised that, amongst other factors, the sensitivity of the respiratory centre to carbon dioxide is dependent upon impulses coming to it from numerous places in the nervous system. The change in sensitivity to carbon dioxide with NREM sleep was thus viewed as being due to a decrease in the number of CNS impulses reaching the respiratory centres. Fink (1961) referred to a "cortical drive" which during wakefulness maintains a substantial volume of ventilation. During periods in which cerebral activity is depressed, such as during natural or anesthetic sleep, the unaugmented cerebral ventilation falls to zero at the carbon dioxide threshold tension and require a suprathreshold increase of carbon dioxide to maintain ventilation. 
In a 1970 review, Plum also referred to a cerebral drive that provides an important component to the volume of breathing during wakefulness. He claimed that the cerebral respiratory activating system is "easily dampened by natural sleep, sedatives, illnesses which dull alertness or produce delirium and neurological illness which produce bilateral structural damage to the hemispheres" (Plum, 1970, p. 166). He also indicated that facilitation of respiration is only provided by a limited number of cerebral points, which are located mainly in the classic somatic motor and pre-motor areas. He went on to comment that in contrast to this relatively limited facilitatory input, large areas of the cerebral hemispheres inhibit respiration. Further, these inhibitory areas comprise most of the hemispheric contribution to the limbic system.

Phillipson (1978) has argued that behavioural influences on respiration should be separated into "state-dependent" and "behaviour-related", and that even in the absence of behavioural ventilatory activities, wakefulness per se has a stimulatory effect on breathing. Phillipson concluded that the state influence depends on the overall degree of mental alertness. This was supported experimentally by Bulow (1963), who reported increases in ventilation associated with moving from a quiet waking to a tense alertness condition occasioned by the performance of complicated mental tasks. Asmussen (1977) emphasised this point, commenting that, "all sensory and intra-cephalic signals that impinge on the reticular formation may play a role in the regulation of respiration" (p. 89).

Sullivan (1980), while supporting the notion of an excitatory wakefulness drive, commented that such a drive should not be confused with "conscious volitional control of respiratory muscles during breath-holding or vocalisation" (p. 222). Although, the characteristic regularity of ventilation in NREM sleep can be explained by the absence of disturbing volitional influences on breathing from higher brain centres (Cherniack, 1981). Several authors have observed that during wakefulness, breathing is governed by both the metabolic and the behavioural respiratory control systems, whereas during 
NREM sleep - and in particular slow wave sleep - it is under sole command of the metabolic system (Cherniack, 1981; Parmeggiani, 1979; Phillipson, 1978; Plum, 1974; Sullivan, 1980). It is generally accepted that the behavioural control system that enables respiratory musculature to be used for non-respiratory purposes such as vocalisation, and to place respiration under voluntary control, arises in cortical and forebrain structures (Plum, 1970).

\subsection{The Relationship between EEG and Measured Respiratory}

\section{Changes}

This review of the literature presents evidence for respiration being under the excitatory influence of a wakefulness drive, a drive which becomes inactive with sleep. In addition, the literature suggests that the ventilatory changes accompanying loss of the wakefulness drive should demonstrate two characteristics. Firstly, they should be systematically related to variations in brain activity. Secondly, because they are mediated by neural mechanisms, they should be relatively rapid.

Studies that have attempted to determine this relationship, have largely only provided anecdotal evidence or general impressions. Bulow (1963) reported a "close linkage between respiration and the level of wakefulness" (p. 97); however, this description remained unquantified. Regarding the aspects of respiration which were found to vary with wakefulness, the author stated that "when the alpha activity in the EEG disappeared and theta waves developed, ventilation decreased" (p. 31). He states that "in contrast to the change in ventilation the change in $\mathrm{P}_{\mathrm{A}} \mathrm{CO}_{2}$ was always more gradual, during the course of some breaths" (p. 31). However, the temporal relationship between ventilation and $\mathrm{P}_{\mathrm{A}} \mathrm{CO}_{2}$ changes, and that between $\mathrm{P}_{\mathrm{A}} \mathrm{CO}_{2}$ and $\mathrm{EEG}$ changes remained unquantified. It should be emphasised that the limitations cited above are largely due to the equipment available at the time of the study, and because of the authors interest being primarily with sleep versus awake differences. 
Naifeh and Kamiya (1981) reported the only attempt to quantify the temporal association of EEG and respiratory changes. In a study using twelve subjects they reported that on average sustained rises in carbon dioxide tension occurred eight seconds before the occurrence of stage one sleep. The range, however, was from 140 seconds before, to 36 seconds after stage-one onset. The method in which the authors defined respiratory decreases casts doubt on the usefulness of the above result. A value for a respiratory variable was recorded during quiet wakefulness (point a), as was its value at the onset of stage one sleep (point b). The change in the variable was defined as occurring when fifty per cent of the a - b difference was attained. Thus the determination of the respiratory change ignored fluctuations in arousal which occur during the transitional stage 1 sleep phase and the respiratory changes could not be specifically linked to arousal level. In addition, the respiratory change was defined in such a way that the change had to occur after the loss of clear wakefulness, but before stage 2 sleep. With these methodological factors considered, results of this study support anecdotal reports that substantial changes occur around sleep onset, but they do not identify the critical events during the transitional phase, nor do they eliminate the possibility of changes during stage 2 sleep.

In an earlier study, Timmons et al. (1972) investigated changes associated with loss of wakefulness that occurred in thoracic and abdominal movements and inspiratory-expiratory phase durations. They reported a "striking parallelism between the transient changes in brain states and respiratory movements" (p. 174). The authors compared the amplitudes of the two breath cycles immediately preceding and following an EEG transition. Transitions of two types were considered: alpha-theta (a drop out of alpha activity of at least ten seconds); and theta-alpha (a subsequent return of alpha for at least five seconds). The data indicated that a reduction of abdominal amplitude occurred with 93 percent of the alpha-theta transitions, with increases associated with 81 percent of theta-alpha transitions. However, thoracic amplitudes were not closely related to 
alpha-theta transitions, so that whether or not ventilation decreased during the EEG transitions, as defined above, remained undetermined.

\subsection{Summary}

Respiration is under behavioural and metabolic control during wakefulness, the behavioural control consisting of both voluntary and state-related elements. During NREM sleep, behavioural control is absent and respiration is controlled solely by metabolic mechanisms. These display an alteration of the set point relating ventilation to the partial pressure of carbon dioxide, as well as, diminished ventilatory responsivess to the stimuli of hypoxia and hypercapnia.There is now evidence to suggest that the variation in the set point and chemosensor responsiveness are due to the removal of the state related behavioral component which can be termed a "wakefulness drive to respiration".

The probable mediation of this wakefulness drive by the ascending reticular activating system leads to the expectation that cortical arousal, as measured by the EEG, will vary systematically with the strength of the drive. Preliminary results suggest that this is the case, and that the cessation of the drive occurs early in the sleep onset period and may be associated in some way with the onset of theta EEG activity. As yet, the exact relationship of the cessation of the wakefulness drive and changes in EEG is unknown. 
CHAPTER FOUR

VENTILATION DURING SLEEP ONSET

IN MALE SUBJECTS 


\section{CHAPTER FOUR}

\section{VENTILATION DURING SLEEP ONSET \\ IN MALE SUBJECTS}

\subsection{Introduction}

The evidence reviewed in the previous chapter demonstrates that ventilation falls during sleep, possibly in close association with sleep onset. This change may be partly due to a fall in metabolic rate and to an increase in upper airway resistance. However it has also been suggested that the reduction in ventilation during sleep may be partly due to the inactivation of a wakefulness drive to respiration. As this inactivation is thought to be mediated by neural mechanisms and linked to arousal, the change in ventilation would be expected to be associated with measures of arousal such as the EEG, and occur rapidly.

The aim of the present thesis is to test this hypothesis. The specific aim of the first experiment is to test the prediction that ventilation decreases in association with changes in arousal state, and to determine if a specific change in arousal state, as indicated by the EEG, is systematically associated with this ventilatory reduction in male subjects.

\subsection{Method}

\subsubsection{Subjects and Design}

Five young male subjects (aged 19 to 23 ) free from respiratory and sleep pathology, each spent two adaptation nights in the laboratory followed by between six and eight nights in which data was collected for analysis. The event of interest in the study was sleep onset and consequently a number of sleep onsets were recorded in each session. Subjects were kept awake, sometimes with the aid of visual (video recorder) or auditory 
(radio) stimulation for approximately thirty minutes and then instructed to go to sleep, at which time the lights and any devices to aid wakefulness were switched off.

After sleep had been achieved and maintained for at least thirty minutes, the subject was re-awakened and the procedure repeated. For analysis purposes each subject was treated as a separate experiment, with each sleep onset as a replication. The number of sleep onsets obtained was $16,20,14,10$, and 15 for D.L., M.L., S.G., M.W., and A.H. respectively. Thus the average number of sleep onsets obtained each night was a little over two. The heights and weights of the subjects were as follows: D.L. $196 \mathrm{~cm}$ and $82 \mathrm{~kg}$; M.L.172 cm and $61 \mathrm{~kg}$; S.G. $178 \mathrm{~cm}$ and $66 \mathrm{~kg}$; M.W. $172 \mathrm{~cm}$ and $66 \mathrm{~kg}$; and A.H. $188 \mathrm{~cm}$ and $92 \mathrm{~kg}$.

\subsubsection{Equipment}

Each subject wore a C.I.G. anaesthetic mask, attached to a Rudolph \#2700 two-way breathing valve. The dead space of the valve was $118.8 \mathrm{ml}$. and, as the mask dead space depended on facial configuration, the total dead space varied between $213 \mathrm{ml}$ and 263 $\mathrm{ml}$. The mask was fitted with an inflatable cuff and was held tightly in place with a head strap. The subjects were trained in the detection of air leaks and were instructed to check for them during the wake periods; the experimenter closely watched the ventilation record during data collection and any transitions in which it was thought that a leak was occurring were aborted land the equipment checked (only three such situations occurred).

An outlet in the breathing valve at the point at which the mask joined the valve led to an Applied Electrochemistry S-3A Oxygen Analyser, via a heating unit and $110 \mathrm{~cm}$. of 1 mm. internal diameter tubing. The rate of expiratory air flow was measured by a Fleisch pneumotachograph connected to the outlet of the breathing valve and a Gould PM 15E pressure transducer. The output signals of both the $\mathrm{O}_{2}$ analyser and the pressure transducer were amplified using a Beckman R411 polygraph recorder and input to a 
D.E.C. PDP-11/23 computer via an eight bit analogue to digital converter. The collected data was stored on either floppy discs or a thirty megabyte Winchester hard disc. Software running under RT11 Pascal was developed in the laboratory to enable the online display, via a high resolution graphics screen, of data entering the computer. In addition, information relating to the integrity of the data collected was available to the experimenter throughout the experimental session via paper chart, a variety of digital volt-meters, and computer alarms.

The oxygen analyser was calibrated to ambient fresh air at the beginning of each session and to known concentration tank air periodically. The subject bedroom was ventilated with fresh air throughout the period of the experimental session and the room heated to between $23^{\circ} \mathrm{C}$ and $28^{\circ} \mathrm{C}$. As mentioned above it was necessary to use either video movies or radio during wake periods to ensure that the subjects remained awake for a sufficient period at the beginning of each sleep onset. Whilst such devices are potential sources of artifact, in that they provide sensory stimulation and increase reticular activity, their use was found to be the least disruptive method of ensuring clear wake activity. Alternatives such as alarms or intercom systems to signal the subjects loss of clear wakefulness were found to produce body movement.

The EEG was recorded via disk electrodes applied to position $\mathrm{C} 3$ or $\mathrm{C} 4$, and a single EOG via electrodes vertically displaced on the outer canthi of the eyes. Both measures were amplified and together with a duplicate of air flow and $\mathrm{O}_{2} \%$ were recorded on paper chart.

\subsubsection{Variables}

Using computer software developed in the laboratory, a number of variables were able to be constructed from the expiratory air flow and $\mathrm{O}_{2} \%$ measures. The variables analysed in the present study were: respiratory cycle duration; the volume of air expired $(\mathrm{Vt})$, corrected to standard temperature and pressure (saturated); fractional end tidal $\mathrm{O}_{2}$ 
level $(\mathrm{FetO} 2)$; minute ventilation (VE); minute $\mathrm{O}_{2}$ consumption $\left(\mathrm{VO}_{2}\right)$; and minute estimates of VE extrapolated from individual breath values. It should be noted that $\mathrm{O}_{2}$ consumption values during non-steady states are inaccurate because of the buffering effects of $\mathrm{O}_{2}$ stores. As a consequence $\mathrm{VO}_{2}$ has only been reported during stable wake and following the attainment of stable sleep (stage 2). $\mathrm{CO}_{2}$ values were not measured as a $\mathrm{CO}_{2}$ analyser was not available.

Sleep state was ascertained for consecutive one minute epochs from the EEG and EOG recordings by an experienced scorer using the standard criteria of Rechtschaffen and Kales (1968). In addition, during particular portions of each sleep onset, the EEG associated with each breath was classified into three categories according to the dominant frequency band: alpha or beta (wake); theta (stage 1); or theta in association with spindles or $\mathrm{K}$ complexes (stage 2). For the purposes of analyisis, "dominant" was defined as the frequency which was present for more than 50 per cent of each breath.

\subsubsection{Data Analysis}

It should be emphasised that each subject was treated as a single case design and analysed independently, with sleep onsets as replications. Thus the data reported below have been presented as averages over sleep onsets within subjects. A second general point is that significance tests have not been conducted. There were two reasons for this. First, the aims of the study were to determine the temporal occurrence, magnitude, and pattern of the respiratory changes at sleep onset; aims not achieved with tests of significance. Second, in each case in which the expected relationship between respiratory variables and the wake/sleep state change was assessed, the respiratory change was in the appropriate direction for virtually all replications in every subject. To indicate the consistency of the observed phenomena, the proportion of replications showing the expected response have been reported where appropriate. Preliminary analyses, and the consistency of the observed effects, each indicated that the changes in ventilatory variables were not dependent on the position within the night, or the time of 
night, of the sleep onsets.

The relationship between respiration and wake/sleep state during sleep onset is difficult to determine, because the transitional phase between wake and the relatively permanent sleep state of stage 2 is characterised by a period of variable duration during which rapid fluctuations in state occur. With this characteristic in mind, five approaches to data reduction, each describing a slightly different aspect of the relationship, were developed. There were a number of reasons for developing five different methods. First, each procedure could only be applied to a sub set of all sleep onsets; second, each procedure was applied to different components of sleep onset; and finally, several of the methods may have been open to influence by confounded variables. As a consequence, any single method was open to criticism. By using a variety of methods of data reduction it was possible to give a comprehensive description of the changes which occurred.

The critical issue assessed by this experiment was the temporal relationship between the reduction in ventilation known to be associated with sleep, and changes in the EEG measure of arousal state. A second issue was the magnitude of the ventilation change, while a third was the extent to which the reduction was due either to changes in cycle duration or Vt. Finally, $\mathrm{O}_{2}$ consumption and $\mathrm{FetO}_{2}$ were assessed to determine if the changes in ventilation were secondary to reductions in metabolic rate. In reporting the results, each method of data reduction has been described and the data relevant to each of these issues presented. 


\subsection{Results}

\section{Method One: Minute values from wakefulness to stage 2 sleep.}

The initial analysis of the data summarised the changes in $\mathrm{VE}, \mathrm{VO}_{2}$, and $\mathrm{FetO}_{2}$ over the total sleep onset period. A sleep onset was identified for analysis if it had ten minutes of unambiguous wake followed by a period of mixed wake-stage 1 which in turn was followed by at least ten minutes of stage 2 . Wake was identified by the presence of alpha, or beta activity, in the EEG, stage 1 by theta activity and stage 2 by theta activity accompanied by spindles and $\mathrm{K}$ complexes. Averages over sleep onsets, using minute values for wake (ten minutes), wake/stage 1 (variable interval) and stage 2 (ten minutes) then showed the general pattern of respiratory activity during sleep onset. The wake/stage 1 period was subjected to a Vincent curve procedure (Vincent, 1912). This allowed changes which occurred over different time intervals to be compared by dividing the variable length periods into a constant number of units. Thus the average values in each quarter of the wake/stage 1 period were determined for each sleep onset.

This analysis demonstrated a substantial reduction in ventilation from wakefulness to stage 2 sleep. The reduction occurred in $100 \%$ of sleep onsets for each subject. The percentage fall varied across the five subjects from 13\% in S.G. to 35\% in M.W. (see table 4.1). A typical example is displayed in figure 4.1. This analysis suggested that the critical segment of the sleep onset period for ventilation changes was the loss of unambiguous wakefulness as defined by the cessation of alpha or beta EEG, rather than the onset of stage 2 . 


\section{Table 4.1}

Respiration during sleep onset : Minute values for male subjects

\begin{tabular}{|c|c|c|c|c|c|c|c|}
\hline \multirow[t]{2}{*}{ Subject } & \multirow{2}{*}{\multicolumn{2}{|c|}{$\begin{array}{c}\text { Wake } \\
\text { (Last } 10 \text { mins) }\end{array}$}} & \multicolumn{4}{|c|}{ Transition in Quartiles } & \multirow{2}{*}{ (First $10 \mathrm{mins}$ ) } \\
\hline & & & 1 & 2 & 3 & 4 & \\
\hline D.L. & VE & 6.77 & 5.63 & 5.00 & 4.91 & 4.29 & 4.45 \\
\hline \multirow[t]{2}{*}{$\mathrm{N}=11$} & $\mathrm{VO}_{2}$ & 0.285 & & & & & 0.275 \\
\hline & $\mathrm{FetO}_{2}$ & 15.97 & 15.65 & 15.13 & 15.03 & 14.77 & 14.79 \\
\hline M.L. & $\mathrm{VE}$ & 3.74 & 3.25 & 3.14 & 3.02 & 2.85 & 2.68 \\
\hline \multirow[t]{2}{*}{$\mathrm{N}=6$} & $\mathrm{VO}_{2}$ & 0.245 & & & & & 0.227 \\
\hline & $\mathrm{FetO}_{2}$ & 14.93 & 14.58 & 14.60 & 14.52 & 14.46 & 13.72 \\
\hline S.G. & VE & 5.65 & 4.96 & 5.11 & 5.05 & 4.95 & 4.93 \\
\hline \multirow[t]{2}{*}{$\mathrm{N}=10$} & $\mathrm{VO}_{2}$ & 0.210 & & & & & 0.205 \\
\hline & $\mathrm{FetO}_{2}$ & 16.01 & 16.05 & 16.04 & 16.00 & 16.07 & 15.62 \\
\hline \multirow{3}{*}{$\begin{array}{l}\text { M.W. } \\
N=7\end{array}$} & VE & 5.13 & 4.56 & 4.49 & 3.98 & 3.71 & 3.35 \\
\hline & $\mathrm{VO}_{2}$ & 0.201 & & & & & 0.162 \\
\hline & $\mathrm{FetO}_{2}$ & 15.97 & 15.88 & 15.66 & 15.60 & 15.70 & 15.42 \\
\hline \multirow{3}{*}{$\begin{array}{l}\text { A.H. } \\
\mathrm{N}=11\end{array}$} & VE & 6.52 & 6.02 & 5.40 & 4.89 & 5.14 & 4.93 \\
\hline & $\mathrm{VO}_{2}$ & 0.345 & & & & & 0.303 \\
\hline & $\mathrm{FetO}_{2}$ & 15.08 & 14.91 & 14.60 & 14.39 & 14.22 & 14.14 \\
\hline
\end{tabular}

Changes in respiratory variables during the final 10 minutes of wake, the transitional period of mixed Wake/Stage 1 sleep and the first 10 minutes of Stage 2 sleep. The variable length transitional period has been divided into quartiles. The reported values are averages over sleep onsets where $\mathrm{N}$ is the number of sleep onsets meeting the criteria (see text). $\mathrm{VE}$ and $\mathrm{VO}_{2}$ are expressed in litres per minute and $\mathrm{FetO}_{2}$ in percentages. See glossary for explanation of abbreviations. 
Figure 4.1

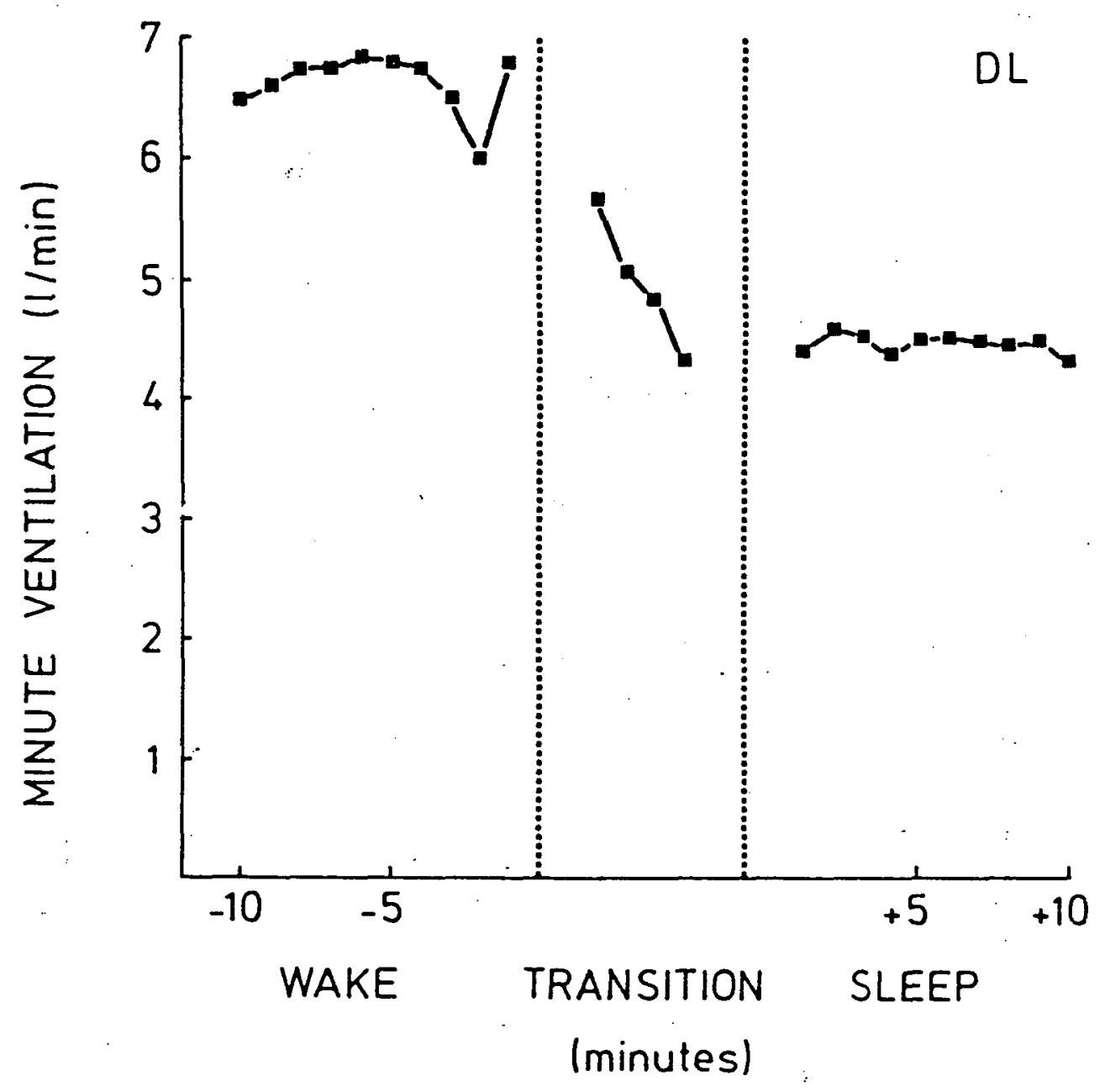

Figure 4.1 Minute ventilation for subject D.L. during the final 10 minutes of wake, the transitional period of mixed Wake/Stage 1 sleep, and the first 10 minutes of Stage 2 sleep. The transition period which varied in length over sleep onsets has been divided into quartiles. The number of sleep onsets averaged was 11 . 
Oxygen consumption was reduced 2\% (S.G.) to $12 \%$ (A.H.) from wake to stage 2 sleep, with the reduction occurring in $84 \%$ of all sleep onsets (ranging from $60 \%$ for S.G. to $100 \%$ for M.L. and A.H.). As was discussed in the method section, consumption values were not calculated during the transition as indirect calorimetry is not a valid measure of energy expenditure in transitional phases, such as a rapid change in ventilation, as blood levels of $\mathrm{O}_{2}$ change less rapidly and thus $\mathrm{O}_{2}$ exchange at the alveoli does not reflect $\mathrm{O}_{2}$ utilization. The data indicated that metabolic rate fell over the sleep onset phase being lower in stage 2 sleep than during wake. However, the reduction in metabolic rate did not account for the total reduction in ventilation as $\mathrm{FetO}_{2}$ also fell in $100 \%$ of sleep onsets for each subject (see table 4.1 ).

In summary, sleep onset was associated with a relatively large reduction in ventilation, which appeared to begin early in the transitional phase. This reduction was partly due to a reduction in metabolic rate and partly a non-metabolic reduction in ventilatory drive.

\section{Method Two: The relationship between significant changes in ventilation and the EEG.}

The next technique was also designed to identify the critical events over the total transition from wake to stage 2 sleep. In this case the analysis reversed the previous procedure and identified significant reductions in ventilation which were then compared with transition points in the EEG. The ventilatory variable used was individual breaths extrapolated to minute values (extrapolated VE). These values were smoothed by computing ten point moving averages. Significant reductions in these values were then identified by two rules: first, if a reduction from one average to the next occurred for nine of ten averages; and second, if a series of consecutive reductions in extrapolated VE showed a total change greater than one third of the change from wake to stage 2 sleep. 
For this analysis a more restricted component of each sleep onset was used. This was the last two minutes of stable wake (though this period was shorter if body movements occurred within the two minutes), the transitional phase characterised by stage 1 sleep and recurrent brief arousals; and the first two minutes of stage 2 sleep. In one subject (M.W.), who had frequent awakening from stage 2 sleep before sustained sleep was achieved, the number of these transitions exceed the number of sleep onsets. Within this time the extrapolated VE was determined for each breath and the EEG during each respiratory cycle, was classified as beta/alpha, theta, or theta with spindles or $\mathrm{K}$ complexes (Stage 2 sleep). After applying the rules to identify significant ventilatory reductions, the beginnings of these changes were compared with the EEG classifications. The critical issue was whether the ventilatory reduction showed a systematic association with either a change from beta/alpha to theta, or from theta to Stage 2 . The data showed that the majority of ventilatory reductions occurred in close proximity to beta/alpha - theta transitions.

Initial inspection of the data in all subjects indicated that ventilatory reductions could be classified into two groups. The largest group (approximately 70\%) were those that clustered around the beta/alpha to theta transitions and occurred within one minute of them, (see table 4.2). In these the change in ventilation occurred on average 3.5 seconds before theta activity commenced. The range was from 12.9 seconds before for M.L. to 3.87 seconds after for S.G. The remainder were not in close proximity to any change in the EEG stage. The unrelated changes were defined as those that were more than one minute from a change in EEG state $(11 \%)$, those that followed an earlier ventilation decrease without an intervening EEG shift (4\%), or those which occurred well into Stage 2 sleep and in all cases, these followed an earlier decrease associated with an alpha/theta shift (15\%). 


\section{Table 4.2}

The relationship during sleep onset between significant decreases in ventilation and EEG activity in male subjects.

$\begin{array}{cccccc}\text { Subject } & \text { Total Number } & \text { Alpha/Theta } & \text { Interval between } & \text { Secondary } & \text { Unrelated } \\ \text { of Decreases } & \text { Related } & \text { EEG Change and } & \text { Alpha/Theta } & \text { Decreases } \\ & & \text { Decreases } & \text { Decrease } & \text { Decreases } & \end{array}$

\begin{tabular}{|c|c|c|c|c|}
\hline $\begin{array}{l}\text { D.L. } \\
\mathrm{N}=16\end{array}$ & 40 & 26 & $-6.36(18.63)$ & 12 \\
\hline $\begin{array}{l}\text { M.L. } \\
\mathrm{N}=16\end{array}$ & 59 & 38 & $-12.90(21.35)$ & 11 \\
\hline $\begin{array}{l}\text { S.G. } \\
N=13\end{array}$ & 43 & 26 & $3.87(23.08)$ & 12 \\
\hline $\begin{array}{l}\text { M.W. } \\
N=16\end{array}$ & 17 & 16 & $-0.67(26.81)$ & 0 \\
\hline $\begin{array}{l}\text { A.H. } \\
\mathrm{N}=14\end{array}$ & 39 & 32 & $-1.51(23.65)$ & 3 \\
\hline
\end{tabular}

The total number of decreases in ventilation were identified according to rules described in the text. Alpha/theta related decreases identifies those occurring within one minute of an EEG change from alpha to theta. The temporal relationship between the EEG change and the reduction in ventilation for these events is shown in the next column. Standard deviations are shown in brackets. Secondary alpha/theta related decreases identified changes which occurred after an earlier decrease without a return of EEG alpha. Unrelated changes were those which did not satisfy either of the above criteria. $\mathrm{N}=$ the number of sleep onsets identified according to the rules described in the text. 
While this analysis indicates that the cessation of alpha activity is the critical event associated with decreased ventilation, the method does not give an accurate estimate of the relationship between the two. This is because the ten point running average procedure introduces a bias towards an early estimate of the timing of the ventilatory change.

\section{Method Three: Correlation analysis between ventilation and the EEG.}

The first two analyses indicated that the loss of alpha activity and the appearance of sustained theta activity in the EEG was the critical event associated with the reduction in ventilation. Further, the data suggested that the ventilatory changes were unrelated to the occurrence of Stage 2 sleep; the usually accepted criterion for sleep onset. This impression was confirmed by a correlational analysis between ventilation and the EEG stages. In this analysis the section of each sleep onset analysed was the same as in the previous method. In addition, the EEG classifications used were the same. The respiratory variables were extrapolated VE, Vt, and cycle duration. For each sleep onset two, point bi-serial correlation coefficients were computed, in which ventilation was the continuous variable and EEG state the discrete variable. In the first, the relationship between ventilation and beta/alpha versus theta EEG activity was assessed, while in the second, the relationship between theta versus Stage 2 sleep was determined. The data for theta activity for the alpha versus theta coefficient were taken from the entire transition as were the data for the theta versus Stage 2 coefficient.

The results of the correlational analysis are shown in table 4.3. Each value is the average coefficient for each subject across sleep onsets. As can be seen the beta/alpha versus theta coefficients range from -0.44 (A.H.) to -0.61 (M.W.). Correlating theta versus Stage 2 changes to the same variable gave much lower coefficients 0.09 (M.W.) to -0.19 (D.L.). This indicates that the change is associated more with the loss of alpha activity than stage 2 sleep. 


\section{Table 4.3}

Average correlations between EEG and respiratory variables during particular phases of sleep onset in male subjects.

\begin{tabular}{|c|c|c|c|c|c|c|}
\hline \multirow[t]{2}{*}{ Subject } & \multicolumn{2}{|c|}{ VE } & \multicolumn{2}{|c|}{$\mathrm{Vt}$} & \multicolumn{2}{|c|}{ Cycle Duration } \\
\hline & $\begin{array}{l}\text { Alpha } \\
\text { theta }\end{array}$ & $\begin{array}{l}\text { Theta } \\
\text { Stage } 2\end{array}$ & $\begin{array}{l}\text { Alpha } \\
\text { theta }\end{array}$ & $\begin{array}{l}\text { Theta } \\
\text { Stage } 2\end{array}$ & $\begin{array}{l}\text { Alpha } \\
\text { theta }\end{array}$ & $\begin{array}{l}\text { Theta } \\
\text { Stage } 2\end{array}$ \\
\hline $\begin{array}{l}\text { D.L. } \\
N=16\end{array}$ & -0.540 & -0.194 & -0.376 & -0.111 & 0.493 & -0.013 \\
\hline $\begin{array}{l}\text { M.L. } \\
N=16\end{array}$ & -0.571 & -0.027 & -0.413 & -0.073 & 0.695 & -0.089 \\
\hline $\begin{array}{l}\text { S.G. } \\
N=13\end{array}$ & -0.527 & 0.044 & -0.494 & 0.037 & -0.313 & 0.058 \\
\hline $\begin{array}{l}\text { M.W. } \\
N=16\end{array}$ & -0.612 & 0.087 & -0.532 & 0.099 & 0.137 & 0.129 \\
\hline $\begin{array}{l}\text { A.H. } \\
N=14\end{array}$ & -0.435 & -0.024 & -0.335 & -0.079 & 0.083 & -0.258 \\
\hline
\end{tabular}

Point bi-serial correlation coefficients between respiration (continuous variable) and EEG state (dichotomous variables). The coefficients were computed for two sets of EEG states, alpha versus theta and theta versus Stage 2 sleep. 
Analysis of $\mathrm{Vt}$ and cycle duration indicated a similar pattern for $\mathrm{Vt}$ as for extrapolated VE, while cycle duration varied markedly across subjects in the extent to which it was related to the beta/alpha versus theta state. Correlating Vt with beta/alpha to theta changes gave coefficients ranging from -0.34 (A.H.) to -0.53 (M.W.). However, correlating Vt to theta - stage 2 changes gave a range of coefficients from 0.10 (M.W.) to -0.11 (D.L.). Thus, as for minute ventilation, the change occurred in each subject with the loss of wake activity. Cycle duration was correlated with the beta/alpha versus theta state in two subjects: D.L. (0.49) and M.L. (0.69). This analysis shows that the change in ventilation was associated with a reduction in Vt in all subjects while cycle duration systematically increased in only two subjects.

\section{Method Four: Breath by breath values at alpha-theta transitions.}

In order to more closely examine the relationship between ventilation and the cessation of alpha activity, smooth and relatively rapid transitions into stage 2 sleep were identified. The formal definition of these transitions was that there be at least one minute of sustained wake activity which was followed by stage 1 theta activity, and eventually stage 2 sleep without rearousals intervening. Transitions were also accepted if one brief period of alpha (less than five seconds) occurred during the stage 1 phase. Having identified these transitions, the data analysed was the last ten breaths before and the first 35 breaths following the onset of theta activity. The data was then averaged across transitions within subjects in a manner analogous to Averaged Evoked Responses. The variables were: extrapolated VE; Vt; cycle duration and $\mathrm{FetO}_{2}$. As noted above, $\mathrm{O}_{2}$ consumption values are not meaningful during transitional states. 
Table 4.4

Breath by breath changes in respiratory variables immediately before and after the transition from EEG alpha/beta to theta activity in male subjects.

\begin{tabular}{|c|c|c|c|c|c|c|}
\hline \multirow[t]{2}{*}{ Subject } & \multicolumn{2}{|r|}{ Alpha } & \multicolumn{4}{|c|}{ Breaths following cessation of alpha } \\
\hline & VE(Extrap) & 6.912 & $\begin{array}{l}1-5 \\
3.917\end{array}$ & $\begin{array}{l}6-10 \\
5.130\end{array}$ & $\begin{array}{l}11-20 \\
4.317\end{array}$ & $\begin{array}{l}21-35 \\
4.374\end{array}$ \\
\hline D.L. & $\mathrm{Vt}$ & 0.377 & 0.241 & 0.274 & 0.264 & 0.264 \\
\hline \multirow[t]{2}{*}{$\mathrm{N}=7$} & $\mathrm{CD}$ & 3.221 & 3.630 & 3.511 & 3.686 & 3.711 \\
\hline & $\mathrm{FetO}_{2}$ & 15.57 & 15.24 & 14.94 & 14.81 & 14.60 \\
\hline \multirow{4}{*}{$\begin{array}{l}\text { M.L. } \\
N=9\end{array}$} & VE(Extrap) & 4.025 & 2.726 & 2.606 & 2.968 & 2.769 \\
\hline & $\mathrm{Vt}_{\mathrm{t}}$ & 0.247 & 0.186 & 0.179 & 0.190 & 0.187 \\
\hline & $\mathrm{CD}$ & 3.675 & 4.266 & 4.091 & 3.996 & 4.155 \\
\hline & $\mathrm{FetO}_{2}$ & 14.92 & 14.75 & 14.79 & 14.77 & 14.39 \\
\hline \multirow{4}{*}{$\begin{array}{l}S . \mathrm{G} \\
\mathrm{N}=3\end{array}$} & VE(Extrap) & 5.492 & 4.205 & 4.153 & 3.969 & 4.141 \\
\hline & $\mathrm{Vt}$ & 0.377 & 0.271 & 0.258 & 0.252 & 0.271 \\
\hline & $\mathrm{CD}$ & 4.125 & 3.813 & 3.792 & 3.773 & 3.924 \\
\hline & $\mathrm{FetO}_{2}$ & 16.61 & 16.65 & 16.71 & 16.60 & 16.39 \\
\hline \multirow{4}{*}{$\begin{array}{l}\text { M.W. } \\
N=7\end{array}$} & VE(Extrap) & 5.471 & 4.229 & 3.592 & 3.490 & 3.341 \\
\hline & $\mathrm{Vt}$ & 0.403 & 0.312 & 0.257 & 0.230 & 0.220 \\
\hline & $\mathrm{CD}$ & 4.526 & 4.568 & 4.476 & 4.547 & 4.447 \\
\hline & $\mathrm{FetO}_{2}$ & 15.69 & 15.93 & 16.02 & 15.83 & 15.47 \\
\hline \multirow{4}{*}{$\begin{array}{l}\text { A. } \mathrm{H} \\
\mathrm{N}=8\end{array}$} & VE(Extrap) & 6.568 & 4.666 & 4.352 & 4.271 & 4.826 \\
\hline & Vt & 0.462 & 0.315 & 0.288 & 0.294 & 0.231 \\
\hline & $\mathrm{CD}$ & 4.154 & 4.093 & 4.003 & 4.072 & 4.217 \\
\hline & $\mathrm{FetO}_{2}$ & 14.73 & 14.64 & 14.44 & 14.32 & 13.92 \\
\hline
\end{tabular}

Breath by breath changes in respiratory variables during the transition from sustained alpha activity of at least one minute to sustained theta leading to Stage 2 sleep. The alpha values were averaged over the last 10 breaths before the cessation of alpha while the remaining values were averaged over varying intervals (see table) to a total of 35 breaths following the cessation of alpha activity. $\mathrm{N}=$ the number of transitions available for each subject. VE(Extrap) is expressed in litres/min, $\mathrm{Vt}$ in litres, $\mathrm{CD}$ in seconds and $\mathrm{FetO}_{2}$ in percentages. See glossary for explanation of abbreviations. 
As shown in table 4.4 , there was a decrease in ventilation during stage 1 theta as compared to the average of the wake period. Again, the change occurred in all replications for every subject. In each subject most of the change occurred within the first five breaths and had asymptoted by 15 breaths. Figure 2 shows a typical pattern (subject M.W.). When the breath values of Vt occurring during wake are compared to those in the succeeding theta period a similar pattern of results to those of extrapolated VE was found, with a decrease occurring in all replications for every subject (see figure 4.3). Most of the change occurred in the first five breaths (see table 4.4). An example, again from subject M.W., is dependence of ventilation changes on changes in Vt.

In contrast, cycle duration showed marked variation across subjects. Subjects D.L. and M.L. displayed an increase in duration during theta as compared to an averaged wake period in all replications. Subject S.G. displayed a slight decrease in all replications, M.W. almost no change and A.H. an initial slight decrease which rebounded to become an increase. Fractional end tidal $\mathrm{O}_{2}$ values fell in all replications for all subjects following the cessation of alpha; though as would be expected, the decrease was delayed compared to the fall in extrapolated VE ( see table 4.4).

The results show a rapid reduction in ventilation associated with the cessation of alpha activity and the appearance of sustained stage 1 theta. As noted above, the change occurred in each subject, on each transition into sleep. The response asymptote varied from subject to subject (approximately 3 to 15 breaths following alpha cessation) and the maximum change varied in magnitude from $28 \%$ (S.G.) to $43 \%$ (D.L.) of wake value (as computed from 5 breath averages). The reduced ventilation observed with this method were greater than with method one, indicating that the minimum value observed during these transitions were, in three subjects (D.L., S.G., and A.H.), substantially less than the ventilation during the first ten minutes of stage 2 sleep. The reduction in ventilation was consistently associated with a reduction in $\mathrm{Vt}$ and in some subjects with an increase in cycle duration. In all subjects $\mathrm{FetO}_{2}$ decreased, indicating that the fall in 
ventilation was not entirely due to a reduction in energy expenditure.

\section{Method Five: Breath by breath values at alpha-theta-alpha}

\section{transitions.}

The previous analyses have evaluated various aspects of the transition from wake to stage 2 sleep. The aim of these methods of data reduction was to minimise the effect of the repeated rearousals which characterise the process of going to sleep either by incorporating them into larger time units, or by eliminating them from the data considered. Thus it was possible that the pattern of respiratory changes identified by these methods was limited to those occasions on which sleep was achieved and were not specific to the loss of alpha activity. To evaluate this hypothesis the transitions identified in method two were inspected for alpha - theta - alpha (ATA) events; those in which following entry into stage 1 theta, a rearousal occurred. The sequence of events analysed were formally defined as 10 breaths which were associated with alpha EEG activity, followed by two or more breaths associated with stage 1 theta and finally one or more breaths during the reappearance of alpha activity. For purposes of data reduction the variable theta period was subjected to a Vincent curve procedure (Vincent, 1912) and divided into quartiles and the transitions averaged within subjects as in method four. The variables analysed were extrapolated $\mathrm{VE}, \mathrm{Vt}$, and cycle duration.

Each of these variables confirmed the results of previous analyses in showing the dependence of level of ventilation on the presence of wakefulness as defined by the presence of alpha/beta EEG activity. All subjects displayed a decrease in extrapolated VE, with the onset of theta activity (see table 4.5). With the appearance of alpha activity this trend was reversed with all subjects increasing ventilation. Three subjects increased extrapolated VE to a level slightly higher than the original alpha period. 
The decrease with the cessation of alpha activity occurred in all replications of three subjects (M.W., A.H., and M.L.), in $95 \%$ of replications in D.L. and $70 \%$ of replications in S.G. The rise associated with rearousal occurred in 100\% (A.H., D.L.), 95\% (M.L.) and 57\% (M.W.) of replications. The lower proportion of replications showing this effect is most likely due to the small number of breaths in the theta phase and alpha rearousal phase in some transitions (as low as two and one breaths respectively).

Table 4.5 also shows the results for the analysis of Vt. This displays a similar pattern to that of extrapolated VE. The decrease with the cessation of alpha activity occurred in all replications in A.H., 95\% in D.L., 92\% in S.G., 91\% in M.L. and 86\% in M.W. The results for respiratory cycle duration also indicate that the relationship of cycle duration to changes in EEG state is variable between subjects. Two subjects show an increase in cycle duration with the cessation of alpha activity (M.L. 95\%, D.L. 90\%), followed by a decrease in duration upon rearousal (M.L. 88\%, D.L. 79\%). One subject (S.G.) shows the opposite pattern of decreasing duration with alpha cessation (77\%) and increasing it with rearousal (77\%). A.H. shows a decrease with alpha cessation (91\%) with a further decrease upon rearousal (82\%). M.W. shows an increase with alpha cessation $(57 \%)$ and a further increase with rearousal (86\%). 
Figure 4.2

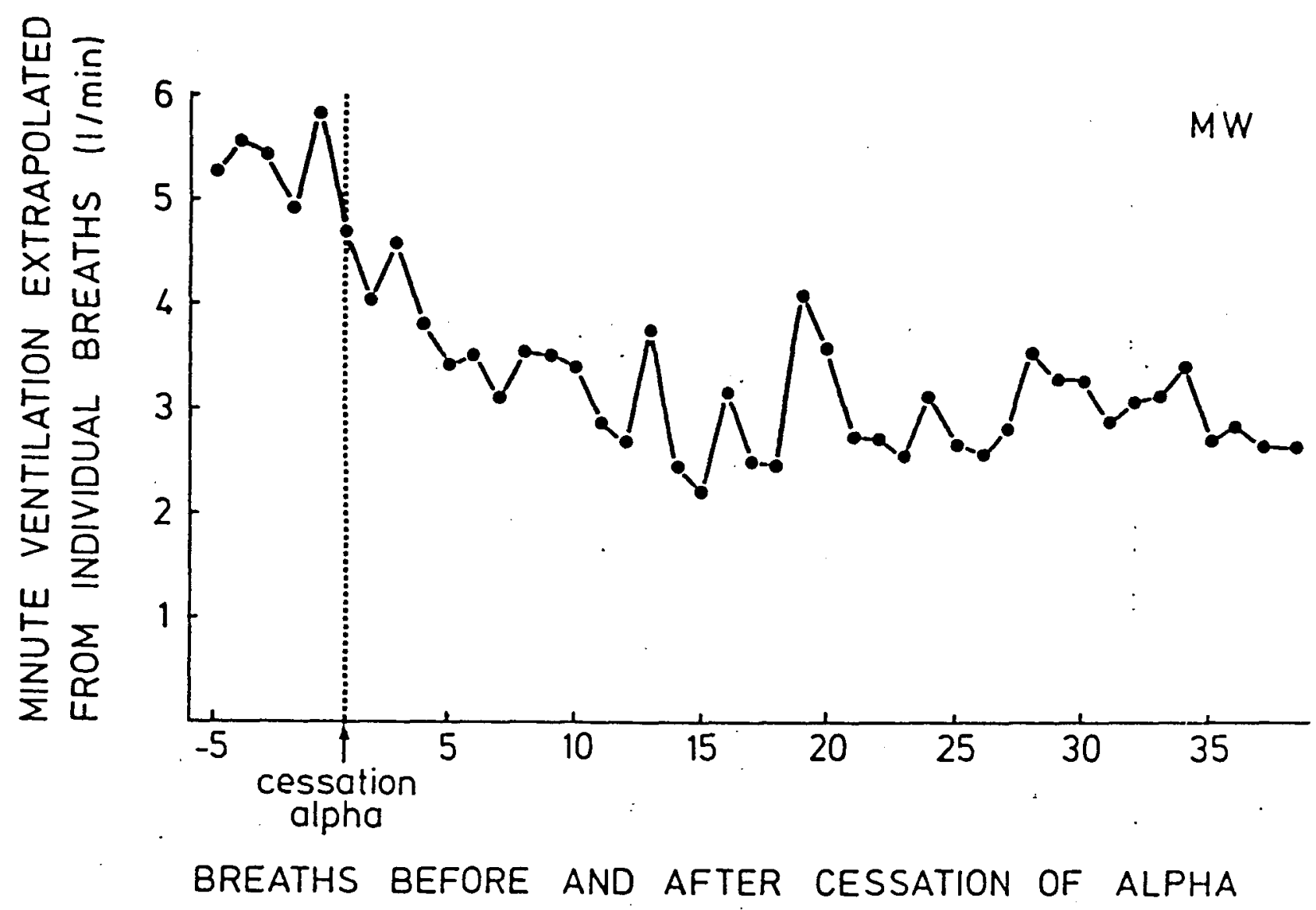

Figure 4.2 Minute ventilation extrapolated from individual breaths for subject M.W. The values for the final 5 breaths before cessation of alpha in the EEG, and the following 35 breaths are shown. The number of events averaged was 7 . The method of defining these transitions is given in the text (method four). 
Figure 4.3
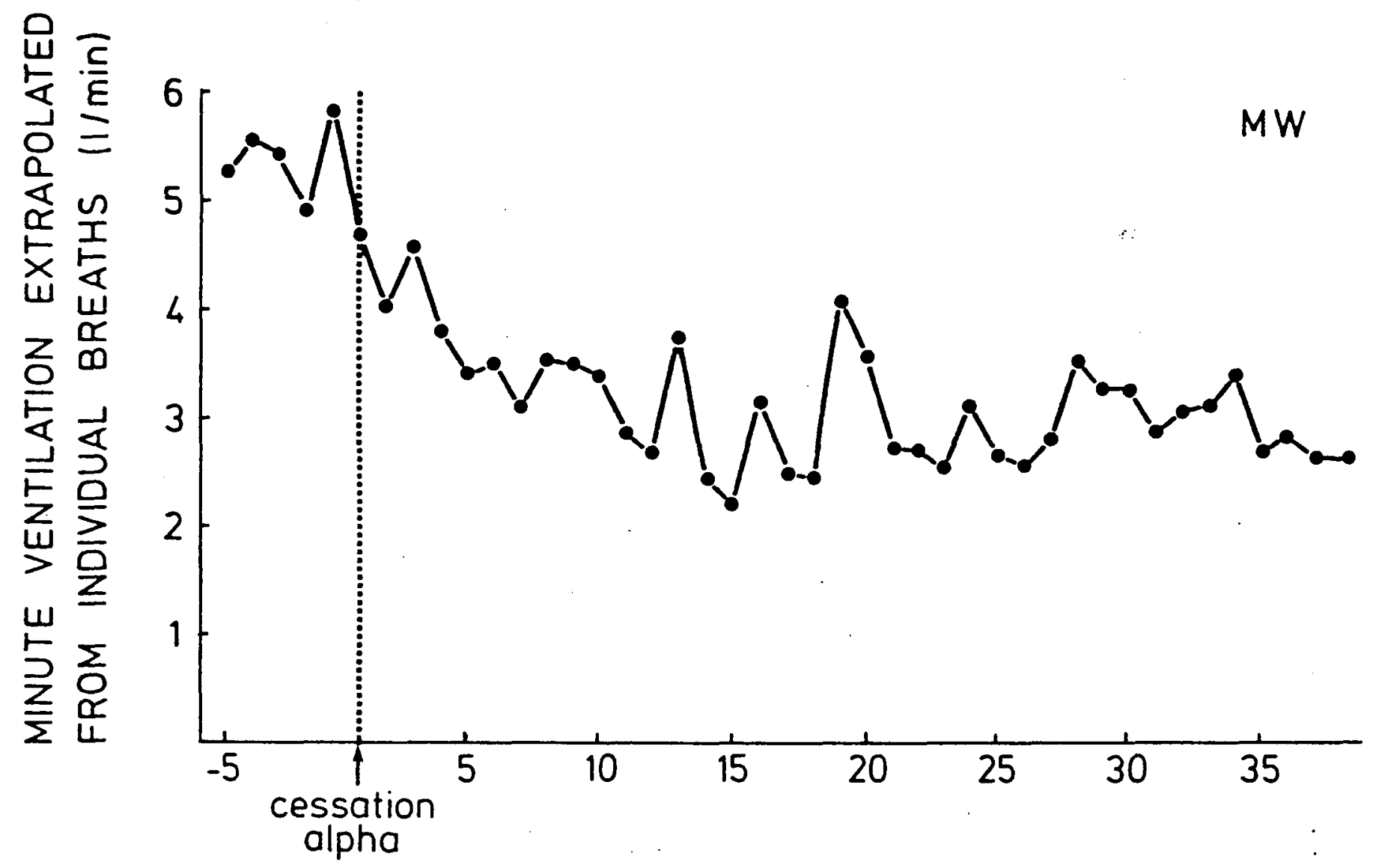

BREATHS BEFORE AND AFTER CESSATION OF ALPHA

Figure 4.3 Tidal volume for subject M.W. The values of the final 5 breaths before cessation of alpha in EEG, and the following 35 breaths are shown. The number of events averaged was 7 . The method of defining these transitions is given in the text (method four). 


\section{Table 4.5}

Breath by breath changes in respiratory variables in alpha-theta-alpha transitions for male subjects.

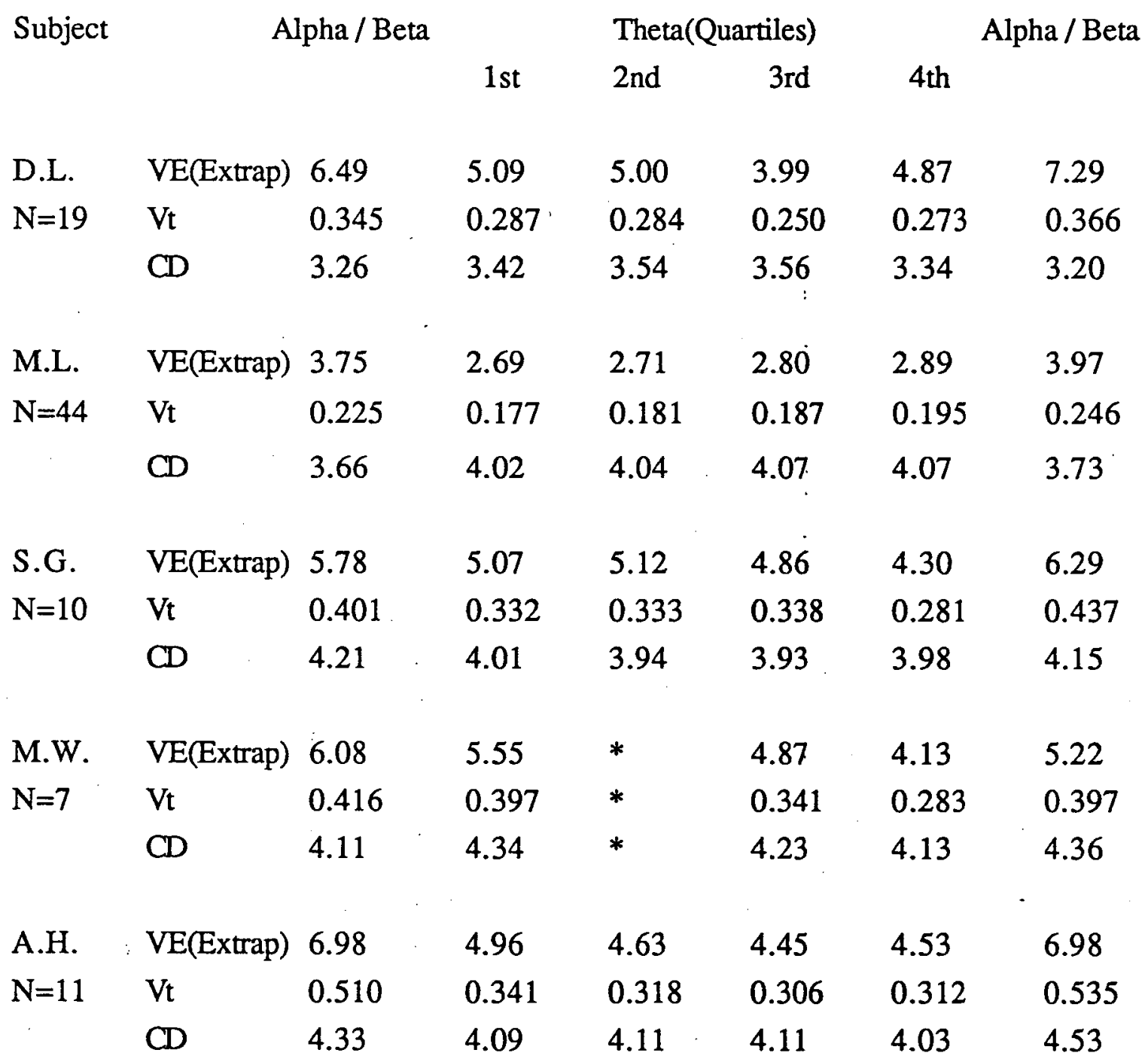

Respiratory changes over alpha-theta-alpha (ATA) events. The initial alpha phase was averaged of the last 10 breaths before the cessation of alpha activity. The theta phase was the period of theta before rearousal and the return of EEG alpha. As this period was variable it has been variable reported as quartiles, with the exception of M.W. whose data is presented as thirds. The final alpha phase was averaged over 2 to 10 breaths, the number varying in different ATA's. $\mathrm{N}=$ the number of ATA's for each subject. $\mathrm{VE}$ (Extrap) is expressed in litres/minute, $\mathrm{Vt}$ in litres and CD in seconds. See glossary for explanation of abbreviations. 


\subsection{Discussion}

In the present experiment the loss of alpha activity in the EEG during sleep onset was closely associated with a substantial, rapid and highly predictable reduction in ventilation. The reduced ventilation was achieved by a reduction in $\mathrm{Vt}$ in all subjects, though in two, cycle duration also increased. Oxygen consumption fell over the total sleep onset period (wake to stage 2 sleep) though $\mathrm{FetO}_{2}$ also fell, indicating that part of the reduction in ventilation was due to non-metabolic factors. It is speculated that the non-metabolic component may reflect the loss of wakefulness drive to ventilation.

The reduction in ventilation at sleep onset is consistent with informal observations reported in earlier papers (Bulow, 1963), though the present study is the first to quantify the magnitude and temporal properties of the phenomenon. The data is also consistent with changes in $\mathrm{FetCO}_{2}$ and abdominal/thoracic contributions to ventilation which have been reported to occur at sleep onset (Naifeh \& Kamiya, 1981, Ogilvie \& Wilkinson, 1984). These studies indicate a close association between central nervous system states, as indicated by the EEG and the respiratory changes; suggesting an interaction between neural structures controlling sleep and those controlling ventilation. Neurological evidence based on recordings from brain stem structures known to be involved in respiratory control is in agreement with this conclusion (Orem et al., 1974, Orem \& Dement, 1976). These studies indicate that there are neurons which lose their association with respiration with the establishment of NREM sleep and others which decrease their level of activity.

In conclusion, the prediction made in the introduction was able to be confirmed. Ventilation was found to decrease systematically in association with a specific change in arousal state. The EEG change was found to be the onset of theta activity. 
CHAPTER FIVE

THE EVIDENCE FOR SEX DIFFERENCES IN RESPIRATION 


\section{CHAPTER FIVE}

\section{THE EVIDENCE FOR SEX DIFFERENCES IN RESPIRATION}

Most studies that have investigated respiration during NREM sleep, including those reported in the previous chapter, have concentrated on male subjects, only a few have reported data collected from females. Nonetheless, there appear to be a number of sex related differences, both in respiration while awake, and in the incidence of sleep related respiratory dysfunction. This chapter reviews the literature describing female ventilation during NREM sleep and presents evidence for sex differences in the control of respiration. It also reviews evidence that progesterone acts as a respiratory stimulant and as the cause of these sex differences.

\subsection{Ventilation During NREM Sleep in Females.}

A small number of studies have reported ventilation associated with NREM sleep in female subjects. On the basis of these it would appear that there is little or no difference between the sexes in this state. As with males, minute ventilation (Douglas et al., 1982a; Gleeson et al. 1986; Krieger et al., 1983; White, 1986; and White, Douglas, Pickett, Weil \& Zwillich, 1983), and tidal volume (Douglas et al., 1982a; White, 1986; and White et al., 1983) are lower during sleep than wakefulness. In comparison, respiratory frequency has shown little or no change with sleep (White, 1986; and White et al., 1983), or an increase (Douglas et al., 1982a). The decrease in ventilation is associated with an increase in the partial pressure of carbon dioxide (Douglas et al., 1982a; Naifeh \& Kamiya, 1981; and White, 1986). 
As mentioned above, there appears to be little, or no difference between the sexes in ventilation during NREM sleep. Both males and females demonstrate a reduced minute ventilation and an increased $\mathrm{PCO}_{2}$ while asleep. The evidence of a sex related difference in the magnitude of the response is difficult to determine as there is some doubt about the most appropriate way of adjusting ventilation measurements to account for differences in body size.

\subsection{Sex Differences in Chemical Control Mechanisms.}

\subsubsection{Awake Differences}

Five studies have reported the hypercapnic ventilatory response to be greater in males than in females when the comparison is made during wakefulness (Berthon-Jones \& Sullivan, 1984; Irsigler, 1976; Patrick \& Howard, 1972; Saunders, Heilpurn \& Rebuck, 1972; and White et al., 1983). All these studies reported males to have a greater slope of the ventilation $/ \mathrm{PCO}_{2}$ curve, and all but Saunders et al. (1972) also found them to have an increased intercept value. The magnitude of the sex difference varies greatly across studies however. Berthon-Jones \& Sullivan (1984) reported the male response to be almost double that of females while awake, whereas two other studies failed to find any sex difference (Douglas et al., 1982c; Hirshman, McCullough \& Weil 1975).

There is less data available on the hypoxic ventilatory drive and once again the results vary across studies. As with the hypercapnic response, Hirshman et al. (1975) reported no sex differences in the response to hypoxia while awake, however, this could be a function of the relatively small size of the female sample. Douglas et al. (1982b) and White et al. (1983) reported a stronger male response while awake. Finally, Berthon-Jones \& Sullivan (1982) reported a tendency for a stronger male response, but did not attempt to analyse sex differences statistically as the sample sizes were too small. 


\subsubsection{Sleep Related Changes}

Two studies have analysed the extent to which the hypercapnic ventilatory response decreases with sleep in the two sexes. Douglas et al. (1982c) found no sex related difference, whereas Berthon-Jones \& Sullivan (1984) found that while all male subjects displayed a decrease in the response with sleep, three of the four females failed to do so. Newsom-Davis et al., (1978), while not reporting awake levels, also reported females as having a greater response than males while asleep. The implications of this last study are unclear however, as it involved the measurement of inspired, rather than end-tidal $\mathrm{PCO}_{2}$.

The effects of NREM sleep on the hypoxic ventilatory response is also uncertain. Berthon-Jones \& Sullivan (1982) reported that both males and females displayed a decrease in the hypoxic response during NREM sleep. However when White et al., (1982) compared the data from six females to that from ten males collected in an identical study (Douglas et al., 1982b), they demonstrated that whereas the male response decreased from wakefulness to sleep the female response did not change.

Another index of respiratory drive is the pressure that can be generated against an occluded airway (mouth occlusion pressure). White (1986) found striking sex differences in the variation of this pressure between wakefulness and sleep. He reported that males displayed a significant increase in occlusion pressure from wakefulness to NREM sleep despite falling ventilation. Females, however, had a significantly higher occlusion pressure than men during wakefulness but had little change during sleep, despite, once again, a decreased level of ventilation. 
The reduction in the ventilatory responses to hypoxia and hypercapnia with sleep have previously been mentioned as evidence for a wakefulness drive to respiration. Mouth occlusion pressure has been viewed as an index of central respiratory drive (White, 1986). Thus sex related differences in the variation of the blood gas responses, in addition to differences in the effects of sleep on occlusion pressure, could indicate that differences between males and females exist in the degree to which breathing is influenced by a wakefulness drive.

\subsection{Evidence for Sex Differences in Sleep Related Respiratory}

\section{Dysfunction.}

There is evidence to suggest that males show a greater incidence of apnea, hypopnea and oxygen desaturation events during sleep. Guilleminault, van der Hoed \& Mitler (1978) reviewed sixty cases from a total of one hundred and fifty apnea sufferers referred to the Stanford clinic over a six year period. Of those reviewed, forty-eight of the fifty obstructive apneics and all ten central apneics were male.

In a study involving asymptomatic subjects, Block et al. (1979), found that males had a significantly greater incidence of hypopnea and oxygen desaturation than women. This result was later confirmed in a population of morbidly obese subjects where abnormal breathing during sleep occurred in six of the seven male subjects but in none of the females (Harman, Wynne, Block \& Malloy-Fisher, 1981). In both the above studies the presence of circulating progesterone in the females was hypothesised as the factor responsible for the sex difference. This hypothesis was supported in a third study which reported a greater incidence of disordered breathing during sleep in post-menopausal women when compared to younger controls (Block, Wynne \& Boysen, 1980). However, the natural physical changes accompanying the ageing process has also been related to an increase in the probability of sleep related respiratory dysfunction in both sexes. Krieger et al. (1983), found pathological respiratory events to be more common in elderly subjects of both sexes, with a non-significant trend for more 
events in male subjects. No sex difference was found in younger subjects. This was similar to the pattern of results reported by Catterall, Calverley, Shapiro, Flenley \& Douglas (1985), which failed to demonstrate an effect of sex but did find significantly more events in older subjects.

Interesting results have come from a series of studies designed to investigate the effects of alcohol on the incidence of sleep-related respiratory pathology. Taason, Block, Boysen, Wynne, White \& Lindsey (1981) reported that administering alcohol before bed was associated with a significant increase in the number of apneas and desaturation events in male subjects. However, Block, Hellard \& Slayton (1985) found no such effect in post-menopausal women. In a study investigating old and young subjects of both sexes, Block, Hellard \& Slayton (1986) found a clear effect of sex. Both male groups were affected by alcohol, while neither group of females demonstrated a significant effect of alcohol. However, post-menopausal women demonstrated a higher incidence of events than younger women, following both alcohol and placebo administration. A separate study which isolated the effects of alcohol to the genioglossus muscle, found effects of alcohol in all male subjects but in only half the females investigated (Krol, Knuth \& Bartlett, 1984).

The literature indicates that while sex-related differences are not the only factor in sleep related respiratory disorders, they are still likely to be a factor. This suggests that some differences in respiratory control during sleep are sex-related. 


\subsection{Evidence for Progesterone as a Respiratory Stimulant.}

Most of the authors reporting sex related differences in respiratory function, awake or asleep, have speculated that they are caused by the presence of higher levels of progesterone in female subjects during the luteal phase of the menstrual cycle. Similarly, differences within female subjects have often been ascribed to the same cause.

The human menstrual cycle can be divided into two phases: the follicular phase during which a single follicle and ovum develop to full maturity, and the luteal phase during which the corpus luteum is the active ovarian structure (Vander, Sherman \& Luciano, 1975, p. 441). In practice these equate to pre-ovulatory and post-ovulatory phases. The corpus luteum is fully functioning and producing maximal quantities of progesterone by the fifth day after ovulation. However, unless the ovum is fertilized and implants within seven days of ovulation, the corpus luteum degenerates and estrogen and progesterone secretion is reduced (Llewellyn-Jones, 1970, p.59). Progesterone levels therefore demonstrate a peak for just a few days around the time of ovulation. For the remainder of the luteal phase, levels are lower than the peak but still higher than those measured in the follicular phase (Botella-Llusiá, 1973, p.53).

Evidence for progesterone acting as a respiratory stimulant comes from four areas of experimentation: the comparison of luteal and follicular phases of the menstrual cycle; the comparison of pregnant (in particular third trimester) and non-pregnant women; the administration of progesterone or synthetic homologues to clinical populations, both male and female; and the administration of progesterone to normal males and experimental animals. Each of these areas have been considered separately in the following sections. 


\subsubsection{Luteal-follicular comparisons.}

Studies of female ventilation during wakefulness have reported higher levels of minute ventilation during the luteal phase of the menstrual cycle (Schoene, Robertson, Pierson \& Peterson, 1981; Stoney, Langer \& Gelling, 1986; Takano, 1984; Takano, Sakai \& Iida, 1981). In addition, Leiter, Doble, Knuth \& Bartlett (1987) and White et al. (1983) reported a non-significant trend for higher luteal ventilation. Associated with the increase in ventilation is a decrease in carbon dioxide partial pressure (England \& Farhi, 1976; Machida, 1981; Schoene et al., 1981; Stoney et al., 1986; Takano, 1984; Takano et al., 1981; White et al., 1983), with Leiter et al.(1987) reporting a non-significant trend in the same direction.

The ventilatory responses to hypercapnia (Leiter et al., 1987; Schoene et al., 1981; Takano et al., 1981) and hypoxia (Schoene et al., 1981; Takano, 1984; White et al., 1983) are both more sensitive during the luteal phase, although White et al. (1982) failed to find a phase difference in the hypoxic ventilatory response during wakefulness. Other respiratory variables have been reported in a small number of studies. Oxygen partial pressure was found to increase during the luteal phase in two studies, (Machida, 1981; Takano, 1984) and White et al. (1983) observed a non-significant trend in the same direction. Stoney et al. (1986) found metabolic rate to be elevated as measured by both oxygen consumption and carbon dioxide production in the luteal phase. However, White et al. (1983) found no effect of phase for either of these variables, while Takano et al. (1981) reported no phase effect for carbon dioxide production.

There is less data available on menstrual effects on breathing during sleep.The hypoxic ventilatory response was found to be similar for the two phases during slow wave sleep and REM sleep, although the luteal response was significantly higher ( $\mathrm{p}<$ 0.05) during stage two sleep (White et al., 1983). In another study, increased oxygen saturation was weakly associated with low levels of progesterone (Stahl, Orr \& Males, 1985). 


\subsubsection{Respiration During Pregnancy}

Progesterone levels are progressively elevated throughout pregnancy (Greig, Coyle, Copper \& Walker, 1962), end-gestational levels being more than twenty times greater than those evidenced in the luteal phase of a normal menstrual cycle (Machida, 1981). The results of studies comparing the respiration of pregnant women to that of non-pregnant controls - being either other women or the same subjects post-partumare similar to those found in menstrual phase experiments. Pregnancy is associated with increased levels of ventilation (Cugell, Frank, Gaensler \& Badger, 1953; Moore, McCullough \& Weil, 1987; Pernoll, Metcalfe, Korach, Wachtel \& Dunham, 1975; Plass \& Oberst, 1983), decreased carbon dioxide partial pressure (Hellegers, Metcalfe, Huckabee, Prytowski, Meschia \& Barron, 1961; Moore et al., 1987; Pernoll et al., 1975; Machida, 1981), and increased oxygen partial pressures (Hellegers et al., 1961; Moore et al., 1987; Machida, 1981). Chemical respiratory control is also altered during wakefulness, with increased ventilatory responsiveness to hypercapnia (Lyons \& Antonio, 1959; Moore et al., 1987; Pernoll et al., 1975) and hypoxia (Moore et al., 1987). All of the above results involved awake subjects.

The association of the respiratory changes listed above with elevated progesterone was demonstrated by Machida (1981). In this study, non-pregnant women were investigated in both the follicular and luteal phases of the menstrual cycle and pregnant women during the first and second halves of gestation. Carbon dioxide partial pressure was found to be inversely correlated with log serum progesterone concentration with a linear correlation ratio of -0.944 . Thus, as progesterone levels increased, $\mathrm{PaCO}_{2}$ decreased. A direct relationship between progesterone and $\mathrm{PO}_{2}$ was also demonstrated, although not evaluated statistically. 


\subsubsection{Progesterone as Therapy for Respiratory Dysfunction.}

A variety of clinical improvements have been demonstrated in patients suffering from: polycythemia, chronic obstructive pulmonary disease, pulmonary emphysema, obesity, and apnea following the administration of progesterone, or the synthetic progestogen, medroxy progesterone acetate (M.P.A.). Ventilation has been increased (Cullen, Brum \& Reidt, 1959; Lyons \& Huang, 1968; Tyler, 1960), along with the partial pressure of oxygen (Kryger, McCullough, Collins, Scoggin, Weil \& Grover, 1978b; Lyons \& Huang, 1968; Morrison \& Goldman, 1979; Sutton, Zwillich, Creagh, Pierson \& Weil, 1975; Orr, Imes \& Martin, 1979) and oxy-haemoglobin saturation (Kryger et al. 1978b; Lyons \& Huang, 1968). In addition, carbon dioxide levels have consistently decreased (Kryger et al. 1978b; Lyons \& Huang, 1968; Morrison \& Goldman, 1979; Sutton et al., 1975; Tyler, 1960).

The above results were all obtained in patients measured while awake. Effects in sleeping patients are less consistent. Oxy-haemoglobin saturation was found to be elevated in sleeping polycythemia sufferers ( Kryger, Glas, Jackson, McCullough, Collins, Scoggin, Grover \& Weil, 1978a). Further, Strohl, Hensley, Saunders, Scharf, Brown \& Ingram (1981), have claimed that $\mathrm{PCO}_{2}$ and $\mathrm{PO}_{2}$ levels improved, and the incidence of apnea decreased, in apneic patients following progesterone therapy. However, Hensley, Saunders \& Strohl (1980) did not observe a significant decrease in the incidence of apnea, while Orr et al. (1979), did not report any effect on $\mathrm{PO}_{2}$ level during sleep despite improvements while awake.

\subsubsection{Effects in Normal Males and Experimental Animals}

In normal males progesterone administration has increased ventilation (Schoene, Pierson, Lackshminaryan, Shrader \& Butler, 1980; Skatrud, Dempsey \& Kaiser, 1978; Zwillich, Natalino, Sutton \& Weil, 1978) and decreased carbon dioxide partial pressure (Goodland, Reynolds, McCoord \& Pommerenke, 1953; Schoene et al., 1980; Skatrud et al., 1978; Zwillich et al., 1978). Other effects include increased hypercapnic and 
hypoxic ventilatory responsiveness (Schoene et al., 1980; Zwillich et al., 1978). Although Goodland et al. (1953) reported increased metabolic rate, Zwillich et al. (1978) did not.

The results of animal studies have generally been consistent with the human literature. Progesterone appears to prevent the appearance of the effects of induced emphysema in rats (Ito \& Aviado, 1968), decreased airway resistance in cats (St. John, Bartlett, Knuth, Knuth \& Daubenspeck, 1986) and to increase ventilation (and the hypercapnic ventilatory response) and to decrease $\mathrm{PCO}_{2}$ in goats (Chiodi \& Snow, 1971).

The hypothesis that sex differences are solely due to females having higher levels of circulating progesterone is insufficient to explain all of the data. Such an hypothesis would predict that pre-menopausal females would have higher levels of ventilation and stronger ventilatory responses to hypoxia and hypercapnia than males. As indicated in section 5.2.1, when differences are found, they are often the opposite of this prediction.

\subsubsection{Mechanism of Action}

Lurie \& Weiss (1967) failed to find progesterone increases in cerebro spinal fluid corresponding to those in peripheral blood in pregnant women. This often quoted study has been used to argue for an effective blood-brain barrier to progesterone. If this is the case, the alterations in chemosensitivity associated with increased progesterone levels would be due to the hormone acting on peripheral chemoreceptor mechanisms. The validity of this conclusion has been called into question by three papers published a decade after the Lurie \& Weiss study. 
Firstly, Tok \&Loesche (1975) applied progesterone directly to the ventral medullary surface of cats, and reported that the carbon dioxide ventilatory response curve moved to the left. Their conclusion was that progesterone acts on medullary chemosensitivity structures to diminish the threshold to carbon dioxide". Secondly, Backstrom, Carstensen \& Södergård (1976) reported a correlation of 0.913 between plasma and cerebro spinal fluid progesterone concentrations ( $p<0.0005$ ). Thirdly, Mei, Gort \& Kao (1977), in an elegant study involving cross-circulating carotid lesioned and intact dogs, reported that progesterone did not act directly on the carotid body, but through a central mechanism.

From the above evidence it would appear that progesterone acts via central carbon dioxide chemoreceptors. However, only three studies have yet been reported and more research is required before a definitive answer is available.

\subsection{The Role of Testosterone in Sex Differences}

Three recent papers address the respiratory effects of androgenous hormones in an attempt to provide further information to explain sex differences. In general, these studies suggest that testosterone contributes to male respiratory chemosensitivity. Sandblom, Matsumoto, Schoene, Lee, Giblin, Bremner \& Pierson (1983) induced obstructive apnea in an otherwise asymptomatic thirty-six-year-old male by administering testosterone. Johnson, Anch \& Remmers (1984) replicated this result with androgen administration to an asymptomatic fifty-four-year-old female. Somewhat paradoxically, in addition to the increased incidence of apnea reported in the Sandblom et al. (1983), study there was an increase in the hypoxic ventilatory response. This latter result was also reported by White ,Schneider, Santeen, McDermott, Pickett, Zwillich \& Weil (1985a) in a study involving testosterone administration to hypogonadal males. The authors of this last study comment that the hypogonadal males had pre-treatment hypoxic ventilatory response levels similar to those reported for females, the implication being that the sex difference in this response was the result of elevated testosterone in males. 
This hypothesis is weakened by the lack of change in the hypercapnic response.

\subsection{Summary}

Females demonstrate decreases in ventilation and increases in carbon dioxide partial pressure associated with NREM sleep in a similar manner to males. There appear to be a number of differences between female and male subjects, however, in terms of waking respiration, chemical control mechanisms during sleep, and in the incidence of sleep-related respiratory dysfunction. It has often been hypothesised that these differences reflect the fact that females have higher levels of circulating progesterone, especially during the luteal phase of the menstrual cycle. Naturally occurring variations of the progesterone level in females, associated with the luteal phase of the menstrual cycle and with pregnancy, have demonstrated a systematic and positive relationship to ventilation and ventilatory responsiveness to hypoxia and hypercapnia. Similar results have been demonstrated with the administration of progesterone to males. Further, the administration of progesterone or a like substance has been effective in the treatment of a variety of respiratory disorders. It is thus sensible to conclude that progesterone acts as a respiratory stimulant. Testosterone, however, also appears to effect respiration by increasing respiratory chemosensitivty, although there is much less data on this hormone than on progesterone.

Sex differences in respiration associated with sleep - in particular changes in chemical control mechanisms - and the possibility that progesterone and testosterone may alter the central drive to respiration, raise questions as to whether or not females exhibit the same changes in respiration at sleep onset as males. Further, there is a question as to whether menstrual phase affects the magnitude, or nature of sleep-onset respiratory changes. These questions are evaluated in the following chapter, which presents the results of a study designed to investigate changes in female respiration during the sleep onset period. 
CHAPTER SIX

VENTILATION DURING SLEEP ONSET IN NORMAL FEMALES 


\section{CHAPTER SIX}

\section{VENTILATION DURING SLEEP ONSET \\ IN NORMAL FEMALES}

\subsection{Introduction}

Investigations into changing properties of respiration during sleep in female subjects have provided differing and ambiguous results, particularly those relating to hypoxic and hypercapnic ventilatory responses. Nevertheless, sex differences in the adjustment from wakefulness to sleep, raise the possibility that females do not possess a wakefulness drive, or that it is weaker than in males. In either case, one would not expect ventilation to change in association with EEG measures of sleep onset. The aims of the present experiment were therefore to investigate the changes in respiration occurring in association with EEG changes during sleep onset. Further, so as to assess the role of progesterone at sleep onset, the changes in respiration were assessed in both the luteal and follicular phases of the menstrual cycle.

\subsection{Method}

\subsubsection{Subjects and Design}

Five young female subjects (aged 18 - 22) who were free from respiratory pathology, were normal sleepers, had no menstrual irregularities and who were not on the contraceptive pill were used in the study.Each subject spent two adaptation nights in the laboratory, followed by twelve experimental nights. The experimental phase consisted of three nights in each of the follicular and luteal phases of two menstrual cycles. Subjects recorded their oral temperature every morning for one month preceding 
the commencement of data collection and throughout the time of the experiment. However, the temperature measurements taken by the subjects proved difficult to interpret and did not vary in any obvious manner across the cycles and thus were not used to determine phase. The specific nights were determined by counting days from the onset of menstruation and were days five to eight in the follicular phase and days 19 to 21 in the luteal phase. The heights and weights of the subjects were as follows: Y.B. $177 \mathrm{~cm}$. and $64 \mathrm{Kg}$.; J.B. $161 \mathrm{~cm}$. and 56Kg.; J.S. $168 \mathrm{~cm}$. and $68 \mathrm{Kg}$.; G.O. $160 \mathrm{~cm}$. and $61 \mathrm{Kg}$; K.M. $155.5 \mathrm{~cm}$. and $56.2 \mathrm{Kg}$.

A blood sample was taken from each subject for each experimental night, with the exception of five nights when this proved impossible due to logistical difficulties. In four subjects the samples were taken between 8:30 and 10:30 a.m. the following morning, in the fifth (Y.B.) between 10 p.m. and midnight on the experimental night. The samples were centrifuged and frozen until subjected to a plasma radioimmunoassay carried out by the department of Clinical Chemistry, at the Royal Hobart Hospital.

The event of interest in the study was sleep onset and consequently a number of sleep onsets were recorded on each night. Subjects were put to bed close to their normal bed time and instructed to stay awake for ten to fifteen minutes before attempting to go to sleep. In the previous study (chapter four), male subjects often found it difficult to remain awake for a sufficient time to obtain adequate respiratory measurements. As a consequence visual and auditory stimuli were used to assist the subjects to remain awake. This procedure may have elevated waking ventilation (Asmussen, 1980). In order to remove the possible effects of sensory stimulation, the procedure was varied with female subjects. They were left in the darkened bedroom with no auditory or visual stimulation. After sleep (stage 2) had been achieved and maintained for at least twenty minutes, the subjects were reawakwened, administered a Stanford Sleepiness Scale and allowed to sit in the laboratory talking and listening to the radio. When they reported feeling "more awake" and had moved towards alertness by at least two points on the 
Sleepiness Scale they were put back to bed and the procedure repeated. This increase in alertness enabled them to stay awake long enough for subsequent awake baseline measurements, without the need for sensory stimulation.

\subsubsection{Equipment}

The equipment used in the study was equivalent to that used with male subjects and a detailed description can be found in chapter four. Subjects wore an anesthetic mask attached to a two way breathing valve (Rudolf \#2600) which was smaller than that used in the male subjects. The valve dead space was $33 \mathrm{ml}$. with the total dead space of mask and valve varying between $101 \mathrm{ml}$. and $183.8 \mathrm{ml}$ due to subjects having differing facial configurations. The only other difference between the studies was the addition of a measurement of EMG taken from bilateral recording of the submentalis muscle.

\subsubsection{Variables}

The respiratory variables investigated were: respiratory cycle duration (CD); the volume of air expired $(\mathrm{Vt})$, corrected to S.T.P. (saturated); fractional end tidal $\mathrm{O}_{2}$ level $\left(\mathrm{FetO}_{2}\right)$; minute ventilation (VE); minute $\mathrm{O}_{2}$ consumption $\left(\mathrm{VO}_{2}\right)$; and minute estimation of VE extrapolated from individual breath values.

Sleep state was ascertained for consecutive one minute epochs for the EEG, EOG, and EMG recordings by an experienced scorer using the standard criteria (Rechtshaffen \& Kales, 1968). In addition, during particular portions of each sleep onset, the EEG associated with each breath was visually classified into three categories according to the dominant frequency band: alpha or beta (wake); theta (stage 1) or theta in association with $\mathrm{K}$ complexes or spindles (stage 2). 


\subsubsection{Data Analysis}

The general results across all nights have been treated in the same way as the data from the previous study (chapter four). That is, each subject was treated as a single case design and analysed independently with sleep onsets as replications. Thus the data below have been reported as averages over sleep onsets within subjects. Again, as with the previous study, significance tests were not conducted to assess differences between wakefulness and sleep. However, tests were conducted to assess the differences between the two menstrual phases.

\subsection{Results}

\subsubsection{Progesterone Assay}

Of the five subjects only two, Y.B. and J.B., reliably show the progesterone concentrations expected in days 19 to 21 of the cycles. Subject J.S. had high progesterone levels in the luteal phase in one of the two cycles. The other subjects, G.O. and K.M. demonstrated uncharacteristic menstrual irregularity during the study. It is possible that this was due to the fact that they were both students and were investigated at the end of the academic year, when stress from impending examinations and assignments may have disrupted their cycles. The progesterone assay involved the use of an Amerlex-M Progesterone RIA kit (IM.3200) with a .05 sensitivity of $0.45 \mathrm{nmol} /$ litre. Due to the progesterone findings, the follicular / luteal comparison was only conducted for Y.B. and J.B.

The data collapsed over all nights for each subject was considered first. Five analysis techniques were used, each of which was explained in detail in chapter four. 


\subsubsection{General findings}

Method One: Minute values from wakefulness to stage 2 sleep. The initial analysis of the data summarized the changes in $\mathrm{VE}, \mathrm{VO}_{2}$, and $\mathrm{FetO}_{2}$ over the total sleep onset period. The last five minutes of wakefulness, the period of mixed wake/stage 1 activity and the first ten minutes of (stage 2) sleep were averaged across sleep onsets. The periods of mixed wake/stage 1 activity were subjected to a Vincent curve procedure (Vincent,1915) which allows variable length intervals to be divided into standard units, in this case quartiles. The results of this analysis, demonstrated reductions in VE in each subject from wake to stage 2 sleep (see table 6.1 and figure 6.1), with the decrease occurring in $100 \%$ of transitions in all subjects. The percentage fall varied across subjects from $16 \%$ in G.O. and K.M to $25 \%$ in J.B. (mean = $19 \%$ ). Inspection of table 6.1 indicates that ventilation decreased early in the transition period possibly in association with the onset of theta stage 1 activity. This was a similar pattern of results to that of male subjects where decreases in ventilation varied across subjects between $13 \%$ and $35 \%$ (mean $=27 \%$ ) and occurred in $100 \%$ of transitions (see chapter four). The difference between the sexes was not significant $(t(8)=1.69, p>.05)$. 
Table 6.1

Respiration during sleep onset : Minute values for female subjects.

\begin{tabular}{|c|c|c|c|c|c|c|c|}
\hline & & Wake & & Transitic & in Quar & & Sleep \\
\hline Y.B. & VE & 3.941 & 3.425 & 3.251 & 3.192 & 3.117 & 3.036 \\
\hline $\mathrm{N}=18$ & $\mathrm{VO}_{2}$ & 0.192 & & & & & 0.171 \\
\hline & $\mathrm{FetO}_{2}$ & 15.62 & 15.47 & 15.24 & 15.14 & 15.07 & 14.91 \\
\hline & $\mathrm{VE} / \mathrm{VO}_{2}$ & 20.53 & & & & & 17.75 \\
\hline & & & & & $\because$ & & \\
\hline J.B. & $\mathrm{VE}$ & 4.737 & 4.164 & 3.910 & 3.813 & 3.690 & 3.542 \\
\hline $\mathrm{N}=20$ & $\mathrm{VO}_{2}$ & 0.194 & & & & & 0.172 \\
\hline & $\mathrm{FetO}_{2}$ & 15.17 & 14.88 & 14.71 & 14.60 & 14.54 & 14.34 \\
\hline & $\mathrm{VE} / \mathrm{NO}_{2}$ & 24.42 & & & & & 20.59 \\
\hline J.S. & $\mathrm{VE}$ & 4.619 & 4.184 & 4.106 & 4.077 & 4.008 & 3.888 \\
\hline $\mathrm{N}=25$ & $\mathrm{VO}_{2}$ & 0.213 & & & & & 0.201 \\
\hline & $\mathrm{FetO}_{2}$ & 14.54 & 14.16 & 14.03 & 13.90 & 13.88 & 13.86 \\
\hline & $\mathrm{VE} / \mathrm{VO}_{2}$ & 21.69 & & & & & 19.34 \\
\hline G.O. & $\mathrm{VE}$ & 4.519 & 4.135 & 3.829 & 3.778 & 3.744 & 3.794 \\
\hline $\mathrm{N}=22$ & $\mathrm{VO}_{2}$ & 0.182 & & & & & 0.160 \\
\hline & $\mathrm{FetO}_{2}$ & 15.10 & 14.95 & 14.79 & 14.68 & 14.63 & 14.60 \\
\hline & $\mathrm{VE} / \mathrm{VO}_{2}$ & 24.83 & & & & & 23.71 \\
\hline K.M. & VE & 3.914 & 3.738 & 3.694 & 3.493 & 3.340 & 3.276 \\
\hline$N=19$ & $\mathrm{VO}_{2}$ & 0.160 & & & . & & 0.153 \\
\hline & $\mathrm{FetO}_{2}$ & 14.93 & 14.92 & 14.75 & 14.73 & 14.65 & 14.27 \\
\hline & $\mathrm{VE} / \mathrm{VO}_{2}$ & 24.46 & & & & & 21.41 \\
\hline
\end{tabular}

Changes in respiratory variables during the final 10 minutes of wake, the transitional period of mixed Wake/Stage 1 sleep and the first 10 minutes of Stage 2 sleep. The variable length transitional period has been divided into quartiles. The reported values are averages over sleep onsets where $\mathrm{N}$ is the number of sleep onsets meeting the criteria (see text). $\mathrm{VE}$ and $\mathrm{VO}_{2}$ are expressed in litres per minute and $\mathrm{FetO}_{2}$ in percentages. See glossary for explanation of abbreviations. 


\section{3}

$\mathrm{VO}_{2}$ also decreased from wake to sleep in all subjects with the magnitude ranging from $4 \%$ in K.M. to $12 \%$ in G.O (mean $=9 \%$ ). The percentage incidence of the decreases varied from $78 \%$ of onsets for K.M. to $96 \%$ for Y.B. Again this was a similar pattern to that of males, where they demonstrated a range of $\mathrm{VO} 2$ decreases between $2 \%$ and $19 \%$ (mean $=9 \%$ ), occurring in $84 \%$ of all transitions $(t(8)=0.00, p>.05)$. As indirect calorimetry is not a valid measure of energy expenditure during changes in ventilation, oxygen consumption values were not calculated during the transitional period.The decrease in $\mathrm{FetO}_{2}$ in all subjects indicates that the decrease in ventilation was of greater magnitude than that required by the decrease in metabolic rate (see table 6.1). As with VE, the decrease in $\mathrm{FetO}_{2}$ occurred in $100 \%$ of sleep onsets for all subjects. The mean percentage falls of 4.4 and 5.2 for females and males respectively was not significantly different $(\mathrm{t}(8)=0.57, \mathrm{p}>.05)$.

The level of the ventilatory equivalent $\left(\mathrm{VE} / \mathrm{VO}_{2}\right)$ can be viewed as an index of the extent to which ventilation is determined by metabolic factors. (Higher values indicate a greater non-metabolic component.) The ventilation equivalent fell in every subject from wakefulness to sleep (see table 6.1), with decreases ranging from $5 \%$ (G.O.) to $16 \%$ (Y.B.) (mean $=11 \%$ ). This analysis indicated that ventilation decreased in all sleep onsets for female subjects and was again a similar result to that obtained from the males in the first experiment, who demonstrated decreases in the ventilatory equivalent ranging from $11 \%$ to $32 \%$ (mean $=20 \%$ ). Again the difference was not significant $(t(8)=1.99, p>.05)$. 
Figure 6.1

GO

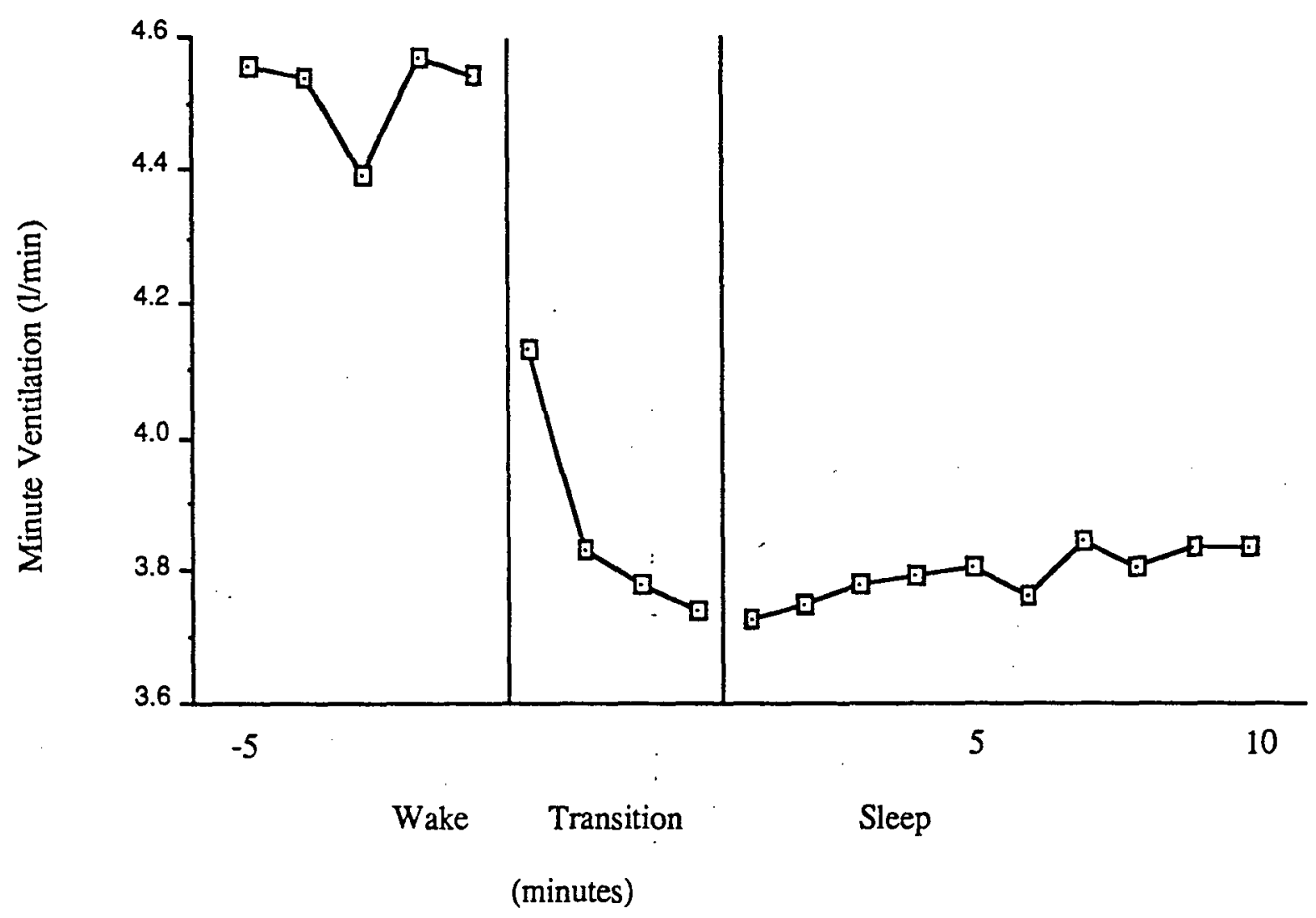

Figure 6.1 Minute ventilation for subject G.O during the final 5 minutes of wake, the transition period of mixed wake/stage 1 sleep, and the first 10 minutes of stage 2 sleep. The transition period, which varied in length over sleep onsets, has been divided into quartiles. The number of onsets averaged was 22 . 
Method Two: The relationship between significant changes in ventilation and the EEG.

The second technique was also designed to identify the critical events over the total transition from wake to stage 2 sleep. In this case the analysis reversed the previous procedure and identified significant reductions in ventilation which were then compared with transition points in the EEG. The critical issue was whether the ventilatory reduction showed a systematic association with either a change from beta/alpha to theta, or from theta to stage 2.

As is displayed in table 6.2 , the largest group of changes $(66 \%)$ in extrapolated ventilation were those which occurred within one minute of an onset of theta activity. In these the change occurred on average 15.46 seconds before the EEG change. The remainder could be considered to fall into one of three categories: those which occurred more than one minute from a change in EEG state(23\%); those which followed an earlier decrease (6\%); or those which occurred well into stage 2 sleep $(5 \%)$. As noted, the average timing of the alpha/theta related changes in ventilation was 15.46 secs. before the EEG change, somewhat earlier than the equivalent value for male subjects which was 3.5 secs. (see chapter four). This difference was statistically significant $(t(8)=3.20, p<.05)$. It should be noted that this technique gives only an approximate temporal relationship between the two variables, and is inaccurate as to the exact timing of events, due to the use of moving averages. Further, as the time associated with an average is that of the first breath of that average, it would be expected that the technique would produce a bias in the direction of anticipating the EEG changes. Nevertheless, these results support those of the first technique in indicating that theta onset, rather than stage 2 onset, is the critical EEG change associated with the majority of ventilation decreases during sleep onset. 
Table 6.2

The relationship during sleep onset between significant decreases

in ventilation and EEG activity in female subjects.

$\begin{array}{llcccc}\text { Subject } & \begin{array}{l}\text { Total number } \\ \text { of decreases }\end{array} & \begin{array}{c}\text { Alpha/Theta } \\ \text { related } \\ \text { decreases }\end{array} & \begin{array}{c}\text { Interval between } \\ \text { EEG change and } \\ \text { decrease }\end{array} & \begin{array}{c}\text { Secondary } \\ \text { alpha/theta } \\ \text { decreases }\end{array} & \begin{array}{c}\text { Unrelated } \\ \text { decreases }\end{array}\end{array}$

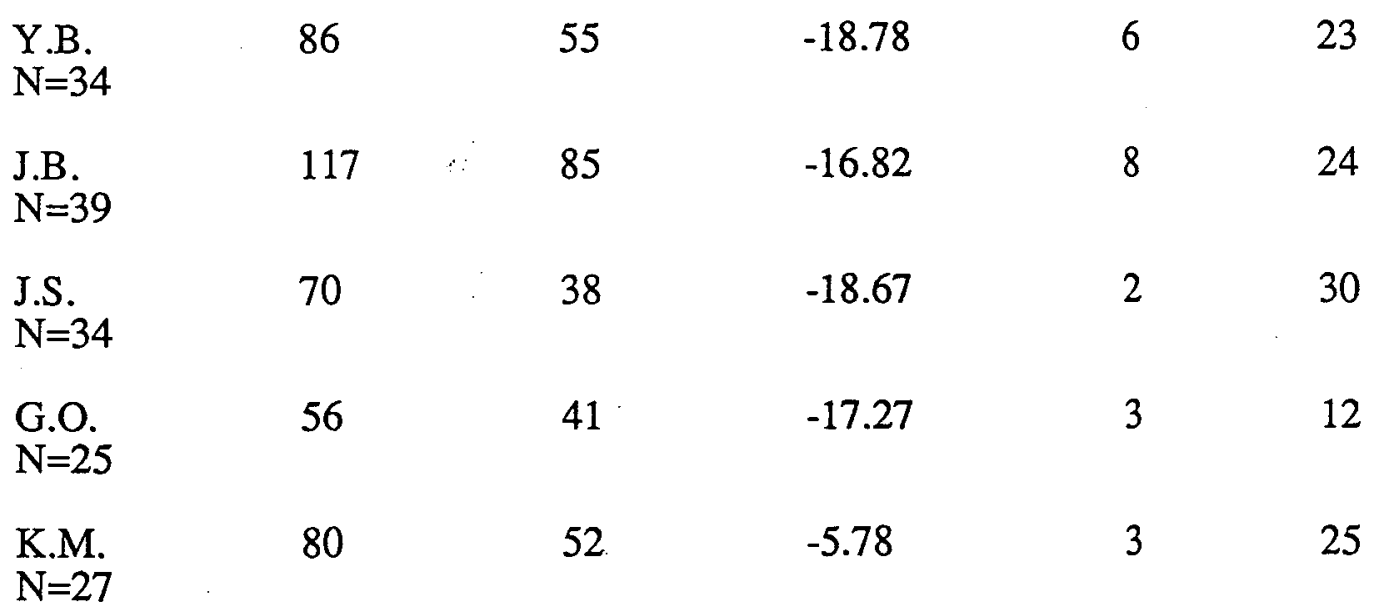

The total number of decreases in ventilation were identified according to rules described in the text. Alpha/theta related identified those occurring within one minute of an EEG change from alpha to theta. The temporal relationship between the EEG change and the reduction in ventilation for these events is shown in the next column. Standard deviations are shown in brackets. Secondary alpha/theta related decreases identified changes which occurred after an earlier decrease without a return of EEG alpha. Unrelated changes were those which did not satisfy either of the above criteria. $N=$ the number of sleep onsets identified according to the rules described in the text. 


\section{Method Three: Correlation analysis between ventilation and the} EEG.

This analysis involved the calculation for each sleep onset, of two, point biserial correlation coefficients. In each analysis, ventilation was the continuous variable and the EEG state the discrete variable. The first analysis determined the relationship between ventilation and beta/alpha versus theta EEG activity, while the second determined the relationship between ventilation and theta versus stage $2 \mathrm{EEG}$. The ventilatory variables used were $\mathrm{VE}$ (Extrap), $\mathrm{Vt}$ and CD.

The results of the regression analysis are shown in table 6.3. Each value is the average coefficient of each subject across sleep onsets. The awake versus theta coefficients for extrapolated ventilation range from -0.45 (G.O.) to -0.50 (Y.B. and J.B.) (mean $=-0.48$ ). The coefficients for theta versus Stage 2 changes were lower -0.09 (Y.B.) to 0.13 (J.B. and K.M.) (mean = 0.06) indicating that the change is associated more with the loss of awake activity than the onset of stage 2 sleep. Analysis of $\mathrm{Vt}$ showed a similar pattern of results. The awake versus theta correlation coefficients ranged from -0.33 (G.O. and K.M) to -0.45 (J.B.) (mean $=-0.36$ ) whereas the theta versus stage 2 coefficients were lower: 0.05 (K.M.) to -0.06 (J.S.) (mean $=-0.04$ ). Changes in $\mathrm{CD}$ in general showed low correlations with both awake versus theta and theta versus stage 2 (see table 6.3). The results were almost identical to those observed in male subjects (see chapter 4). The analysis supported the previous techniques in associating ventilation changes with the onset of theta activity. It also provided evidence that the change in ventilation is primarily mediated by tidal volume rather than $\mathrm{CD}$. 


\section{Table 6.3}

Average correlations between EEG and respiratory variables during particular phases of sleep onset in female subjects.

\section{VE}

Subject

Y.B.

$\mathrm{N}=34$

J.B.

$\mathrm{N}=39$

J.S.

$\mathrm{N}=34$

G.O.

$\mathrm{N}=25$

K.M.

$\mathrm{N}=27$

$-0.488$
$\mathrm{Vt}$

Awake Theta

Theta Stage 2

$-0.340 \quad 0.010$

$-0.453 \quad 0.037$

$-0.364 \quad-0.063$

$-0.333 \quad-0.055$

$-0.320 \quad 0.052$

0.134

0.131

$-0.035$

$-0.011$

\subsection{4}

Cycle Duration

Awake Theta

Theta Stage 2

$0.502-0.137$

$-0.012 \quad-0.251$

$0.203-0.030$

$0.178 \quad-0.068$

$0.293 \quad-0.152$

Point bi-serial correlation coefficients between respiration (continuous variable) and EEG state (dichotomous variables). The coefficients were computed for two sets of EEG states, awake versus theta and theta versus stage 2 sleep. The values presented in this table were averaged over sleep onsets. 


\section{Method Four: Breath by breath values at awake-theta}

\section{transitions.}

In order to more closely examine the relationship between ventilation and the cessation of alpha activity, smooth and relatively rapid transitions from alpha into stage 2 sleep were identified. The data analysed in each transition was the last five breaths before and the first 35 breaths following the onset of theta activity. The data was then averaged across transitions within subjects in a manner analogous to Averaged Evoked Responses. The variables analysed were: extrapolated VE; $\mathrm{Vt} ; \mathrm{CD}$; and $\mathrm{FetO}_{2}$.

There was a decrease in VE (Extrap) from the awake values to those obtained from theta in all subjects (see table 6.4 and figure 6.2). The decrease occurred in $96 \%$ of all transitions. The magnitude of the change varied from $9 \%$ in K.M to $22 \%$ in Y.B. (mean $=15 \%)$, was substantial in all subjects within the first five breaths, and had typically reached an asymptote by fifteen breaths following theta onset. In males, the decrease occurred in $100 \%$ of transition and ranged between $25 \%$ and $39 \% \quad($ mean $=32 \%)$ (see chapter four). While the pattern of the change was the same in both sexes, the magnitude was consistently less in females, the difference being statistically significant $(t(8)=5.00, p<.01)$. As with the male subjects, the VE decrease was again mediated by variations of $\mathrm{Vt}$ in all subjects ranging from $5 \%$ in K.M. to $20 \%$ in Y.B. $($ mean $=12 \%$ ), with the difference between males and females being significantly different $(t(8)=4.11, p<.001)$. However, in two subjects (J.B. and K.M.), the magnitude of the percentage change in cycle duration was comparable with that of Vt. There was not a significant difference between males and females in CD or FetO2 $(\mathrm{t}(8)=0.05, \mathrm{p}>.05$ and $\mathrm{t}(8)=$ $0.00, p_{.}>05$ respectively). The analysis thus confirmed that the critical point associated with the ventilation decrease at sleep onset is theta onset . 


\section{Table 6.4}

Breath by breath changes in respiratory variables immediately before and after the transition from EEG alpha/beta to theta activity.

\begin{tabular}{|c|c|c|c|c|c|c|}
\hline \multirow{2}{*}{\multicolumn{2}{|c|}{ Subject }} & \multirow{3}{*}{$\begin{array}{l}\text { Awake } \\
3.930\end{array}$} & \multicolumn{3}{|c|}{ Breaths following onset of theta } & \multirow{3}{*}{$\begin{array}{l}21-35 \\
3.069\end{array}$} \\
\hline & & & \multirow{2}{*}{$\begin{array}{l}1-5 \\
3.498\end{array}$} & \multirow{2}{*}{$\begin{array}{l}6-10 \\
3.263\end{array}$} & \multirow{2}{*}{$\begin{array}{l}11-20 \\
3.092\end{array}$} & \\
\hline Y.B. & VE(Extrap) & & & & & \\
\hline \multirow[t]{3}{*}{$\mathrm{N}=23$} & $\mathrm{Vt}$ & 0.216 & 0.189 & 0.179 & 0.172 & 0.173 \\
\hline & $\mathrm{CD}$ & 3.297 & 3.231 & 3.284 & 3.350 & 3.378 \\
\hline & $\mathrm{FetO}_{2}$ & 15.21 & 15.26 & 15.29 & 15.18 & 14.93 \\
\hline \multirow{4}{*}{$\begin{array}{l}\text { J.B. } \\
N=21\end{array}$} & VE(Extrap) & 4.268 & 3.923 & 3.939 & 3.693 & 3.657 \\
\hline & $\mathrm{Vt}$ & 0.255 & 0.277 & 0.232 & 0.219 & 0.222 \\
\hline & $\mathrm{CD}$ & 3.313 & 3.494 & 3.559 & 3.595 & 3.668 \\
\hline & $\mathrm{FetO}_{2}$ & 14.99 & 14.82 & 14.72 & 14.64 & 14.48 \\
\hline \multirow{4}{*}{$\begin{array}{l}\mathrm{J} . S . \\
\mathrm{N}=22\end{array}$} & VE (Extrap) & 4.690 & 3.924 & 4.116 & 3.907 & 4.025 \\
\hline & $\mathrm{Vt}$ & 0.351 & 0.307 & 0.320 & 0.307 & 0.315 \\
\hline & $\mathrm{CD}$ & 4.503 & 4.733 & 4.687 & 4.751 & 4.732 \\
\hline & $\mathrm{FetO}_{2}$ & 14.24 & 14.05 & 13.97 & 13.87 & 13.01 \\
\hline \multirow{4}{*}{$\begin{array}{l}\text { G.O. } \\
\mathrm{N}=15\end{array}$} & VE(Extrap) & 4.775 & 4.219 & 4.221 & 4.152 & 4.083 \\
\hline & $\mathrm{Vt}$ & 0.322 & 0.282 & 0.284 & 0.282 & 0.279 \\
\hline & $\mathrm{CD}$ & 4.045 & 4.025 & 4.067 & 4.096 & 4.134 \\
\hline & $\mathrm{FetO}_{2}$ & 15.02 & 14.79 & 14.79 . & 14.68 & 14.56 \\
\hline \multirow{4}{*}{$\begin{array}{l}\text { K.M. } \\
\mathrm{N}=21\end{array}$} & VE(Extrap) & 4.162 & 3.768 & 3.807 & 3.750 & 3.790 \\
\hline & $\mathrm{Vt}$ & 0.268 & 0.244 & 0.255 & 0.247 & 0.254 \\
\hline & $\mathrm{CD}$ & 3.860 & 3.893 & 4.023 & 3.962 & 4.015 \\
\hline & $\mathrm{FetO}_{2}$ & 14.84 & 14.75 & 14.71 & 14.67 & 14.53 \\
\hline
\end{tabular}

Breath by breath changes in respiratory variables during the transition from sustained awake EEG activity to at least one minute of sustained theta leading to stage 2 sleep. The awake values were averaged over the last 10 breaths before the onset of theta while the remaining values were averaged over varying intervals to a total of 35 breaths following the onset of theta activity. $\mathrm{N}=$ the number of transitions available for each subject. $\mathrm{VE}\left(\right.$ Extrap) is expressed in litres/min, $\mathrm{Vt}$ in litres, $\mathrm{CD}$ in seconds and $\mathrm{FetO}_{2}$ in percentages. See glossary for explanation of symbols. 
Figure 6.2

JS

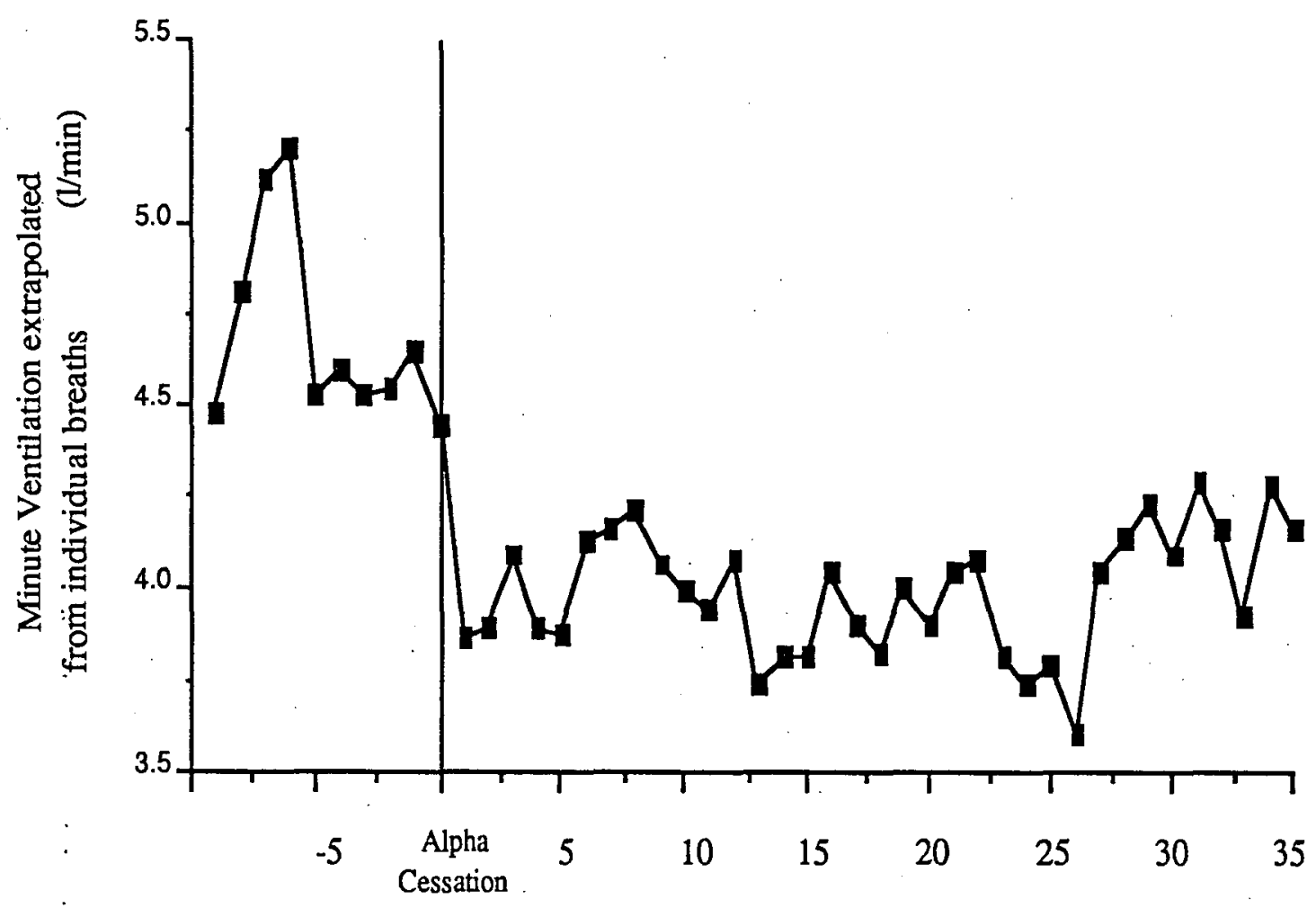

Breaths before and after cessation of Alpha

Figure 6.2 Minute ventilation extrapolated from individual breaths for subject J.S. The values for the final 5 breaths before cessation of alpha in the EEG, and the following 35 breaths are shown. The number of events averaged was $21^{\circ}$. 
Method Five: Breath by breath values at alpha-theta-alpha transitions.

The transitions identified in method two were inspected for alpha - theta alpha events; those in which following entry into stage one theta, a re-arousal occurred. The events analysed contained the last five breaths of beta/alpha, a theta period which was subjected to a Vincent curve procedure and divided into quartiles, and finally one or more breaths associated with the reappearance of beta/alpha. The variables analysed were: VE(Extrap); Vt; and CD.

A decrease in extrapolated VE with the appearance of theta activity occurred in $95 \%$ of all transitions on average varying from $91 \%$ for Y.B to $100 \%$ for J.S. (see table 6.5). A rise with the reappearance of awake EEG activity was only slightly less common (87\%) varying from $81 \%$ of cases for J.B. to $96 \%$ for K.M. Once again Vt displayed a similar pattern of results. The decrease with the onset of theta had an average occurrence of $94 \%$ varying from $89 \%$ of J.B. to $100 \%$ of J.S. and an increase with the reappearance of awake EEG in $86 \%$ of cases varying from $79 \%$ for G.O. to $92 \%$ of K.M. Variations in CD were much less consistent with an average increase with theta onset of $50 \%$ and a re-arousal associated decrease in $51 \%$ of cases. The results for each variable were similar to those obtained for males (see table 4.5). 
Table 6.5

Breath by breath changes in respiratory variables in alpha-theta-alpha transitions for female subjects.

\begin{tabular}{|c|c|c|c|c|c|c|c|}
\hline \multirow{2}{*}{ Subject } & \multicolumn{2}{|c|}{ Alpha / Beta } & \multicolumn{3}{|c|}{ Theta (Quartiles) } & \multicolumn{2}{|r|}{ Alpha / Beta } \\
\hline & & & $1 \mathrm{st}$ & 2 nd & $3 r d$ & 4th & \\
\hline Y.B. & VE(Extrap) & 4.090 & 3.634 & 3.501 & 3.369 & 3.094 & 3.791 \\
\hline \multirow[t]{2}{*}{$\mathrm{N}=36$} & $\mathrm{Vt}$ & 0.230 & 0.199 & 0.188 & 0.180 & 0.168 & 0.213 \\
\hline & $\mathrm{CD}$ & 3.352 & 3.300 & 3.241 & 3.245 & 3.338 & 3.376 \\
\hline J.B. & VE(Extrap) & 4.486 & 4.108 & 3.928 & 3.857 & 3.803 & 4.184 \\
\hline \multirow[t]{2}{*}{$\mathrm{N}=47$} & $\mathrm{Vt}$ & 0.247 & 0.232 & 0.222 & 0.221 & 0.220 & 0.240 \\
\hline & $\mathrm{CD}$ & 3.311 & 3.420 & 3.420 & 3.467 & 3.506 & 3.389 \\
\hline J.S. & VE(Extrap) & 4.355 & 3.873 & 3.867 & 3.752 & 3.680 & 4.251 \\
\hline \multirow[t]{2}{*}{$\mathrm{N}=20$} & $\mathrm{Vt}$ & 0.326 & 0.288 & 0.290 & 0.283 & 0.285 & 0.325 \\
\hline & $\mathrm{CD}$ & 4.568 & 4.510 & 4.583 & 4.566 & 4.670 & 4.598 \\
\hline G.O. & VE(Extrap) & 4.366 & 3.772 & 3.854 & 3.988 & 3.899 & 4.222 \\
\hline \multirow[t]{2}{*}{$\mathrm{N}=24$} & $\mathrm{Vt}$ & 0.299 & 0.262 & 0.273 & 0.268 & 0.262 & 0.290 \\
\hline & $\mathrm{CD}$ & 4.127 & 4.205 & 4.257 & 4.106 & 4.071 & 4.146 \\
\hline K.M. & VE(Extrap) & 3.982 & 3.831 & 3.749 & 3.878 & 3.763 & 4.049 \\
\hline \multirow[t]{2}{*}{$\mathrm{N}=23$} & $\mathrm{Vt}$ & 0.252 & 0.245 & 0.244 & 0.252 & 0.247 & 0.262 \\
\hline & $\mathrm{CD}$ & 3.808 & 3.745 & 3.876 & 3.891 & 3.959 & 3.899 \\
\hline
\end{tabular}

Respiratory changes over alpha-theta-alpha (ATA) events. The initial alpha phase was averaged of the last 10 breaths before the cessation of alpha activity. The theta phase was the period of theta before rearousal and the return of EEG alpha. As this period was variable it has been variable reported as quartiles. The final alpha phase was averaged over 2 to 10 breaths, the number varying in different ATA's. $\mathrm{N}=$ the number of ATA's for each subject. VE(Extrap) is expressed in litres/minute, $V_{t}$ in litres and CD in seconds. See glossary for explanation of abbreviations. 


\subsubsection{Luteal - Follicular Comparison.}

\section{Method One.}

A two factor analysis of variance with repeated measures on each variable, was conducted for each subject using the minute averages from method one, the two factors being arousal state (wake, sleep), and phase (follicular, luteal). Of interest was whether phase had a general effect on ventilation and whether it altered the wake - sleep difference. The data relevant to the main effect of state has, of course, already been presented.The relevant means for the analyses are presented in table 6.6.

The pattern of results were similar for the two subjects. Ventilation was unaffected by menstrual phase $(F(1,32)=0.64, p>.05$ for $Y . B$. and $F(1,36)=$ $0.89, p>.05$ for J.B.), as was the difference in ventilation between wakefulness and sleep $(F(1,32)=0.02, p>.05$ for Y.B and $F(1,36)=0.34$, p>.05 for J.B.). Oxygen consumption tended to be higher in the follicular phase, however the difference was only significant for J.B. $(F(1,32)=1.27$, $p>.05$ for Y.B and $F(1,36)=12.7, p<.01$ for J.B.). The difference between wakefulness and sleep in $\mathrm{O} 2$ consumption was not affected by menstrual phase $(F(1,32)=0.12, p>.05$ for Y.B. and $F(1,36)=0.84, p>.05$ for J.B. $)$. End tidal $\mathrm{O} 2$ differed between phases as a result of the elevated $\mathrm{O} 2$ consumption, though once again the difference was only significant for one of the subjects $(F(1,32)=5.24, p<.05$ for $Y . B$ and $F(1,36)=0.98, p>.05$ for J.B. $)$. The interaction effect for FetO2 was not significant for either subject $(F(1,32)=0.03, p>.05$ for Y.B. and $F(1,36)=0.21, p>.05$ for J.B.). The ventilation equivalent was slightly elevated in the luteal phase (see figure 6.3), but the difference was not significant $(F(1,32)=2 . \dot{9}$, $p>.05$ for Y.B. and $F(1,36)=3.09, p=>.05$ for J.B.) and again the interaction was not significant $(F(1,32)=0.13, p>.05$ for Y.B. and $F(1,36)=0.84, p>.05$ for J.B. $)$. 


\section{Table 6.6}

Respiration during sleep onset : Minute values for follicular and luteal menstrual phases.

Wake Transitions in Quartiles Sleep

$\begin{array}{llllllll}\text { Y.B. } & \mathrm{VE} & 3.984 & 3.609 & 3.324 & 3.202 & 3.197 & 3.099 \\ \text { Luteal } & \mathrm{VO} 2 & 0.188 & & & & & 0.163 \\ \mathrm{~N}=12 & \mathrm{FetO}_{2} & 15.78 & 15.47 & 15.38 & 15.32 & 15.21 & 15.10 \\ & \mathrm{VE} / \mathrm{VO} \mathrm{O}_{2} & 21.21 & & & & & 19.02\end{array}$

$\begin{array}{llllllll}\text { Y.B. } & \mathrm{VE} & 3.854 & 3.056 & 3.104 & 3.096 & 2.790 & 3.012 \\ \text { Follic. } & \mathrm{VO}_{2} & 0.202 & & & & & 0.187 \\ \mathrm{~N}=6 & \mathrm{FetO}_{2} & 15.32 & 15.11 & 14.95 & 14.80 & 14.81 & 14.56 \\ & \mathrm{VE} / \mathrm{VO}_{2} & 19.12 & & & & & 16.03\end{array}$

$\begin{array}{llllllll}\text { J.B. } & \mathrm{VE} & 4.710 & 4.189 & 3.951 & 3.822 & 3.629 & 3.451 \\ \text { Luteal } & \mathrm{VO}_{2} & 0.184 & & & & & 0.163 \\ \mathrm{~N}=12 & \mathrm{FetO}_{2} & 15.24 & 14.90 & 14.82 & 14.73 & 14.60 & 14.36 \\ & \mathrm{VE} / \mathrm{VO}_{2} & 25.54 & & & & & 21.12\end{array}$

$\begin{array}{llllllll}\text { J.B. } & \mathrm{VE} & 4.779 & 4.125 & 3.848 & 3.799 & 3.781 & 3.687 \\ \text { Follic. } & \mathrm{VO}_{2} & 0.208 & & & & & 0.186 \\ \mathrm{~N}=8 & \mathrm{FetO}_{2} & 15.07 & 14.85 & 14.55 & 14.42 & 14.45 & 14.30 \\ & \mathrm{VE} / \mathrm{NO}_{2} & 23.86 & & & & & 20.75\end{array}$

Changes in respiratory variables during the final 10 minutes of wake, the transitional period of mixed Wake/Stage 1 sleep and the first 10 minutes of Stage 2 sleep. The variable length transitional period has been divided into quartiles. The reported values are averages over sleep onsets where $\mathrm{N}$ is the number of sleep onsets meeting the criteria (see text). $\mathrm{VE}$ and $\mathrm{VO}_{2}$ are expressed in litres per minute and $\mathrm{FetO}_{2}$ in percentages. See glossary for explanation of abbreviations. 


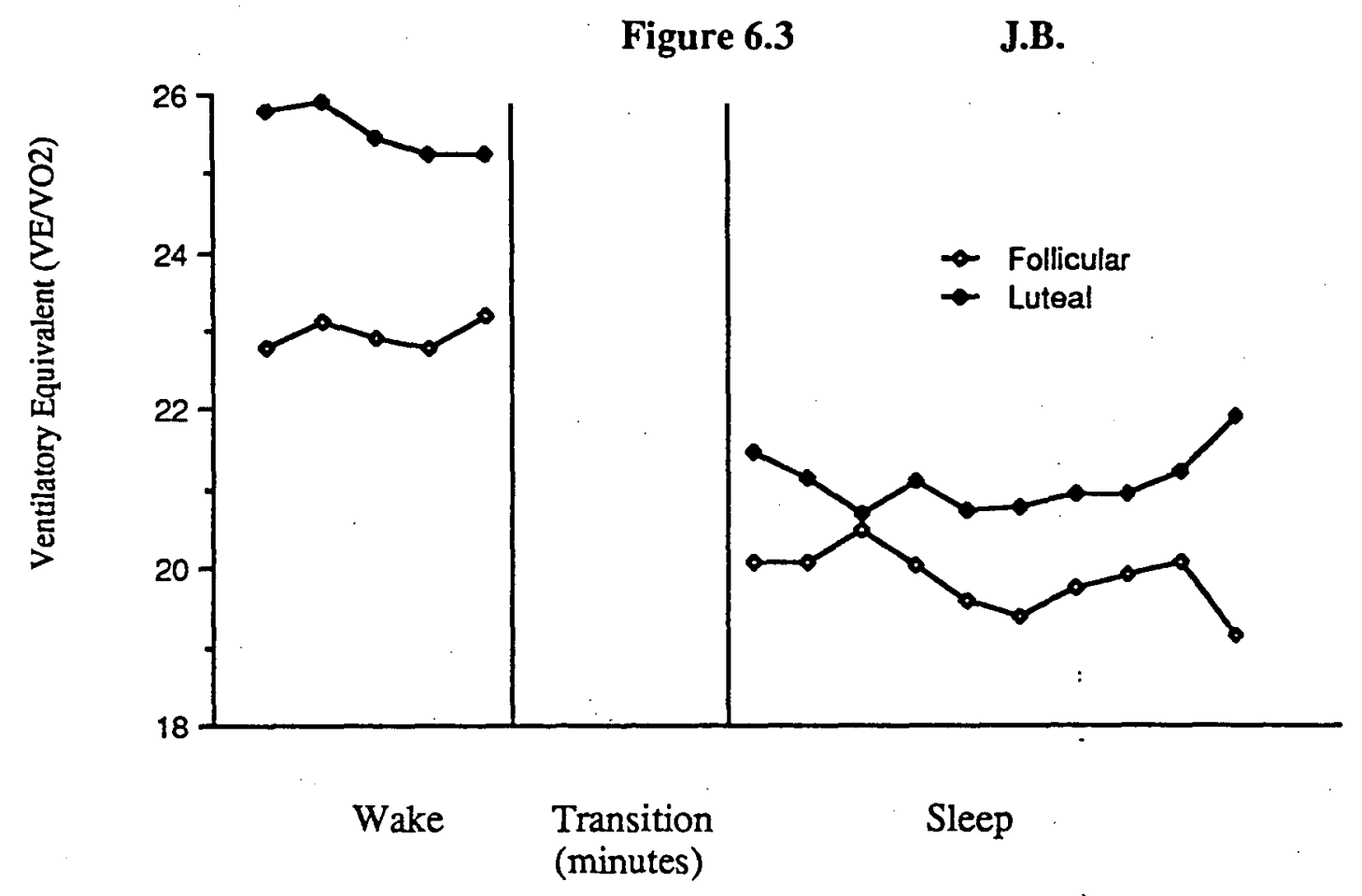

Figure 6.3 The ventilation equivalent for subject J.B. for the luteal and follicular menstrual phases. Data is presented from the last 5 minutes of wake, the transitional period of mixed wake / stage 1 sleep and the first 10 minutes of stage 2 sleep. The transition period which varied in length over sleep onsets has been divided into quartiles. 


\section{Methods Two and Three:}

$t$ tests were conducted on the data from methods two and three to assess the effect of menstrual phase. Both the timing of the respiratory changes relative to the EEG and the point biserial correlation coefficients proved non significant in both subjects. Thus, in both subjects, theta onset was seen as the point of change in the EEG in both the luteal and follicular phases. The results if these analysis are presented in tables 6.7 and 6.8 .

\section{Method Four.}

An analysis of variance was conducted using breath values associated with alpha and theta EEG activity. The alpha breaths used, were the last ten before the onset of theta activity. The theta breaths used were those which occurred between 26 and 35 breaths following theta onset. The relevant means for this analysis are presented in table 6.9.

VE(Extrap) was unaffected by menstrual phase $(F(1,42)=3.39, p>.05)$ for Y.B. however it was higher during the follicular phase $F(1,38)=10.89$, $\mathrm{p}<.01$ ) for J.B. The interaction between menstrual phase and arousal state for $\mathrm{VE}$ (Extrap) was not significant for either subject $(F(1,42)=1.30, p>.05$ for Y.B and $F(1,38)=0.49, p>.05$ for J.B.). An identical pattern of results was evidenced for tidal volume. $V t$ was unaffected by menstrual phase $(F(1,42)=$ $1.26, p>.05)$ for Y.B. but was higher during the follicular phase $F(1,38)=$ $12.32, \mathrm{p}<.01)$ for J.B. Again the interaction between menstrual phase and arousal state was not significant for $\mathrm{Vt}$ for either subject $(F(1,42)=0.02$, $p>$ .05 for $Y . B$ and $F(1,38)=0.79, p>.05$ for J.B.).

Cycle duration was unaffected by menstrual phase $(F(1,42)=0.77, p>.05$ for Y.B. and $F(1,38)=0.33, p>.055$ for J.B.), as was the difference in $C D$ between wakefulness and sleep $(F(1,42)=1.23, p>.05$ for Y.B and $F(1,38)=0.00, p>.05$ for J.B.). End tidal 02 was elevated in the luteal phase 
for Y.B. $(F(1,42)=12.47, p<.01)$ however there was no significant phase effect for J.B. $(F(1,38)=0.84, p>05)$. The interaction effect for FetO2 was not significant for either subject $(F(1,42)=0.46, p>.05$ for Y.B. and $F(1,38)=0.09, p>.05$ for J.B.).

In summary, neither subject demonstrated a significant interaction between phase and state in any variable for either the minute or breath by breath analyses. Thus, the variation between wakefulness and sleep did not differ as a function of menstrual phase. In addition, there were essentially no differences in ventilation between the menstrual phases.The only significant difference showed higher ventilation during the low progesterone, follicular phase. 


\section{Table 6.7}

Summary of the analyses of the timing of ventilatory decreases comparing follicular with luteal phase.

Subject

$$
\text { t }
$$

p

Degrees of Freedom

Y.B.

$$
-0.56
$$

0.576

53

J.B.

$-1.65$

0.103

80

Results of t-tests conducted comparing the temporal relationships between changes in ventilation, and changes in EEG, during sleep onset, in both menstrual phases. See glossary for explantion of abbreviations. 


\section{Table 6.8}

Summary of the analyses of the correlation coefficients between ventilation and EEG state comparing follicular with luteal phase.

\begin{tabular}{|c|c|c|c|c|c|}
\hline Subject & Variable & & $\mathrm{t}$ & $\mathrm{p}$ & Degrees of Freedom \\
\hline \multirow[t]{6}{*}{ Y.B. } & Vt & Alpha-theta & -1.49 & 146 & 37 \\
\hline & $\mathrm{Vt}$ & Theta-Two & -0.89 & .378 & 37 \\
\hline & $\mathrm{CD}$ & Alpha-theta & 1.69 & .100 & 37 \\
\hline & $\mathrm{CD}$ & Theta-Two & 0.40 & .692 & 37 \\
\hline & VE & Alpha-theta & -0.67 & .505 & 37 \\
\hline & $\mathrm{VE}$ & Theta-Two & 0.77 & .445 & 37 \\
\hline \multirow[t]{6}{*}{ J.B. } & $\mathrm{Vt}$ & Alpha-theta & -0.12 & 904 & 32 \\
\hline & $\mathrm{Vt}$ & Theta-Two & -0.15 & .881 & 32 \\
\hline & $\mathrm{CD}$ & Alpha-theta & 0.26 & .797 & 32 \\
\hline & $\mathrm{CD}$ & Theta-Two & -1.14 & .262 & 32 \\
\hline & VE & Alpha-theta & 0.34 & .773 & 32 \\
\hline & VE & Theta-Two & 0.56 & .582 & 32 \\
\hline
\end{tabular}

Results of t-tests conducted to compare the point biserial correlation coefficients produced by method three for both menstrual phases. See glossary for explanation of abbreviations. 
Table 6.9

Breath by breath changes for follicular and luteal menstrual phases.

\begin{tabular}{clllll} 
& & \multicolumn{2}{l}{ Luteal $(\mathrm{N}=13)$} & \multicolumn{2}{c}{ Follicular(N=10) } \\
Subject & & Alpha & Theta & Alpha & Theta \\
Y.B. & VE(Extrap) & 4.123 & 3.049 & 3.884 & 2.993 \\
& Vt & 0.222 & 0.167 & 0.227 & 0.173 \\
& $\mathrm{CD}$ & 3.218 & 3.506 & 3.501 & 3.474 \\
& FetO 2 & 15.30 & 15.03 & 15.13 & 14.78 \\
& & & $\ddots$ & & \\
& & Luteal $(\mathrm{N}=11)$ & Follicular & $(\mathrm{N}=10)$ \\
& & Alpha & Theta & Alpha & Theta \\
J.B. & VE(Extrap) & 4.383 & 3.462 & 4.746 & 3.699 \\
& Vt & 0.240 & 0.213 & 0.261 & 0.266 \\
& $\mathrm{CD}$ & 3.314 & 3.718 & 3.294 & 3.699 \\
& FetO & 14.94 & 14.45 & 14.91 & 14.40
\end{tabular}

Breath by breath changes in respiratory variables during the transition from sustained awake EEG activity to at least one minute of sustained theta leading to stage 2 sleep. The awake values were averaged over the last 10 breaths before the onset of theta while the theta values were averaged over 35 breaths following the onset of theta activity. $\mathrm{N}=$ the number of transitions available for each subject. VE(Extrap) is expressed in litres/min, $\mathrm{Vt}$ in litres, $\mathrm{CD}$ in seconds and $\mathrm{FetO}_{2}$ in percentages. 


\subsection{Discussion}

The data indicate that in female subjects, the change in ventilation during sleep onset is associated with the onset of theta EEG activity. Further, this change is mediated by a decrease in tidal volume with respiratory frequency being variable in its pattern of change. The ventilation decrease was shown to be greater than that required by decreasing metabolic rate as end tidal oxygen levels rose and the ventilation equivalent fell with sleep in all subjects. As was documented statistically in the results section (6.3), the data were consistent with those reported for the male subjects of the first experiment (see chapter four).

There was some suggestion that males and females differed with respect to the timing and magnitude of the ventilatory change. These results must be treated with caution as the comparison of the magnitude and timing of respiratory changes at sleep onset were made on samples collected at different times and with slight differences in methodology. The differences in the timing of the fall in ventilation is also compromised by the use of running averages to smooth the ventilatory data and by the relative difficulty in identifying alpha theta transitions in the female subjects. Nevertheless the data suggest the possibility that the timing of the changes in ventilatory control and brain activity may be slightly different in females, as opposed to males.

The differences in the magnitude of the ventilatory fall at the onset of theta activity revealed in the breath by breath analysis is perhaps of greater interest. The data suggest that either the magnitude of the process affecting the change in ventilation is different, or that male subjects are affected by an additional process. For example, in addition to a loss of wakefulness drive to ventilation, sleep onset may be associated with an increase in airway resistance. Indeed airway resistance has been shown to be higher during NREM sleep than wakefulness (Lopes, Tabachnik, Muller, Levison, Bryan, 
1983). Thus, the fall in ventilation in males may be due to both factors, while only one (eg. the decrease in wakefulness drive) may operate in females.

There are two further factors which should be considered in interpreting the sex differences. First, in order to equate the samples for differences in body size, the change in ventilation over sleep onset has been expressed as a percentage change. However, there is no basis on which to establish the validity of this procedure and the differences between the groups may not be physiologically meaningful. Second, the differences between male and female subjects may have been due to the slightly different procedures used to maintain wakefulness during the awake measurements in each experiment.

Thus the critical finding from this study is that females, like males, have large and rapid reductions in ventilation at sleep onset which are systematically associated with the loss of alpha activity and the appearance of theta in the EEG. As for males, it is proposed that this change in part reflects the loss of a wakefulness drive to respiration during sleep.

Any statements regarding the effect of menstrual phase are limited by the fact that only two subjects were able to be investigated. However, in these two, the ventilation decrease in both phases were of similar magnitude and demonstrated the same degree of consistency and temporal relationship to the onset of theta activity. The data also indicated that oxygen consumption tends to be lower in the luteal phase relative to the follicular in both states whereas ventilation was at about the same level. As a consequence the ventilation equivalent tended to decrease from luteal to follicular phases as well as from wake to sleep. However, these differences were small and not all significant. Further, contradictory differences were also noted.

The evidence for a change in ventilation at sleep onset would appear to be as strong as that previously reported for male subjects, in that both groups seem to show the same pattern of change at the point of theta onset in the EEG. 
Further, it is apparent that the effect is independent of menstrual phase. Neither subject displayed any interaction between wakefulness and sleep and and menstrual phase for any ventilatory variable. The evidence is consistent with the hypothesis that the effect is due to a cessation of a wakefulness drive to ventilation. However, it is possible that the magnitude of the effect and the nature of the critical components, may differ between females and males. 
CHAPTER SEVEN

METHODOLOGICAL CONSIDERATIONS 


\section{CHAPTER SEVEN}

\section{METHODOLOGICAL CONSIDERATIONS}

As with any area of scientific endeavor, it is necessary to determine the extent to which the methodology used in experiments has a systematic effect on the results produced. In the present chapter, three areas of concern from the two previous experiments are discussed. These are: the degree of sensory stimulation present during data collection; the influence of changes in upper airway resistance on the data collected; and the effects of wearing a mask or mouthpiece on the level of ventilation. A review of the studies that have investigated these aspects, is presented together with a discussion the implications of these studies for the data of the present thesis.

\subsection{Sensory Stimulation.}

Before considering the effects of equipment used to measure ventilation, it is worthwhile discussing two papers that have investigated the effects of sensory stimulation on respiration. Asmussen (1977) reported that when subjects closed their eyes after reading, ventilation decreased by between eight and fourteen percent. Shea, Walter, Pelley, Murphy \& Guz (1987), in a more detailed study, investigated the effects of both visual and auditory stimulation on ventilation. There were three levels of visual stimulation; eyes closed with a blindfold, eyes open in the presence of a bright light, and eyes open and reading. The change from eyes closed to the eyes open condition was associated with a six percent increase in both respiratory frequency and minute ventilation. In moving from the eyes open to reading condition, however, there was no further rise in ventilation despite a further increase in respiratory frequency. Thus, the level of ventilation varied with the physical intensity of the stimulus rather than an increase in its cognitive nature. 
There were also three levels of auditory stimulation: silence, white-noise, and listening to verbal communication. Unlike the pattern of results associated with the three analagous visual conditions, ventilation did not show a significant difference between the silence and white-noise conditions. However, moving from white-noise to verbal input, led to a six percent increase in ventilation.

The above studies have clear implications for research investigating the effect of arousal state on ventilation, in that they indicate that ventilation can be increased by various types of sensory stimulation. It is therefore necessary to control the ambient level of sensory stimulation so that, as far as possible, it does not vary systematically with periods when subjects are awake and asleep.

In the first experiment of the present thesis, (chapter four), awake baseline measurements were taken under conditions of moderate auditory and visual stimulation, associated with watching a video or listening to a radio in a dimly lit bed room. After the completion of the awake period, the lights and the radio or video recorder were switched off. Thus during the sleep onset period and NREM sleep, the amount of sensory stimulation was much less than when subjects were awake. It is possible that ventilation decreases could thus have been produced by the decrease in sensory stimulation. However, such an effect could in no way explain the association between ventilation decreases and the onset of theta activity, nor the increase in ventilation associated with the reappearance of alpha/beta activity reported in method five. Nonetheless, in the second study (chapter six), care was taken to ensure that data collection was conducted under identical conditions of minimal sensory stimulation both while the subjects were awake and while they were asleep.That is, the bedroom was dark, and the subjects had their eyes closed during wakefulness as well as while they were asleep. 


\subsection{Upper-airway Resistance}

It is possible that the decreased level of ventilation during NREM sleep is due to an increase in airway resistance, rather than a reduction in ventilatory drive. Upper airway resistance is known to increase during NREM sleep in humans (Hudgel et al., 1984; Lopes et al., 1983) and animals (Megirian \& Sherrey, 1980; Megirian et al., 1978; Orem \& Lydic, 1978; Orem et al., 1977b; Sherrey \& Megirian, 1977; and Sherrey \& Megirian, 1980). However, Rist et al. (1986) have shown that during sleep, reduced mechanical efficiency accounts for only a portion of the total reduction in ventilation, the other factors being reduced central respiratory neural output and reduced metabolic rate. Further, there is evidence that in animals ventilation decreases occur with sleep, despite the maintenance of a patent airway (Phillipson et al., 1976; Phillipson et al., 1978; and Sullivan et al., 1978). It remains to be determined whether or not increased airway resistance occurs during the period of sleep onset in humans and to what extent it can account for the ventilation decrease during this time.

\subsection{Effects of Apparatus}

Four studies have provided evidence that when subjects wear a mouthpiece and nose-clip, ventilation is affected during wakefulness. Three studies have reported increases in minute ventilation as a result of increased tidal volume, with no change in respiratory frequency: (Askanazi, Silverberg, Foster, Hyman, Milic-Emili \& Kinney, 1980; Sackner, Nixon, Davis, Atkins \& Sackner, 1980; Weissman, Askanazi, Milic-Emili \& Kinney, 1984). Both Askanazi et al.(1980) and Weissman et al. (1984) reported that increased tidal volume was due to an increase in inspiratory flow rate. However, Sackner et al. (1980) found this to be the case only during exercise, there being no effect on inspiratory flow rate when the subjects were at rest. 
Gilbert et al., (1972) also reported a significantly increased tidal volume associated with the use of a mouthpiece and nose-clip. However, in this experiment there was no effect on minute ventilation, as the tidal volume increase was balanced by a significant decrease in respiratory frequency. This pattern of results was reported whether or not a valve was attached to the mouthpiece; so the authors considered it to be independent of equipment dead space. They interpretted the decrease in respiratory frequency as being due to stimulation of the nasal mucosa by the nose-clip, with the increase in tidal volume being a secondary effect required to maintain ventilation at the resting level. In contrast, Sackner et al. (1980) reported increased levels of ventilation associated with increased equipment dead-space. They suggested that the increased tidal volume associated with wearing a mask and nose-clip, can be explained by "switching the entry of air from the much smaller dead-space of the nasal cavity to the larger dead-space of the buccal cavity" (p. 938).

Weissman et al. (1984) performed two manipulations in an attempt to determine the primary factor affecting ventilation. Firstly, they used a nose-clip with a mouthpiece half the original size. This manipulation did not affect tidal volume, respiratory frequency, or minute ventilation. In the second manipulation a nose-clip alone was used. The results were that tidal volume displayed a non-significant increase, respiratory frequency decreased significantly and minute ventilation remained unchanged. The authors concluded that the combined results supported the hypothesis that increased minute ventilation was due to the stimulation of the oral region provided by the mouthpiece, and that once again the respiration rate was decreased by the nose-clip.

Only two studies have considered the effects of wearing a mask to measure ventilation instead of a mouthpiece and nose-clip. Askanazi et al. (1980) reported that the mask had the same effect as the mouthpiece, with increased tidal volume and the same respiratory frequency. However, the magnitude of the change in tidal volume was twice 
as large for the mask. However contradictory results were reported by Hirsch \& Bishop (1982). They found that tidal volume was significantly less (by twenty five percent), and respiratory frequency significantly faster (by thirty five percent) when a mask, as opposed to a mouthpiece and nose clip, was used. Minute ventilaion and end-tidal carbon dioxide levels were unchanged. Two other facts relating to air breathing were revealed by the study. First, breathing frequency while wearing a mask was similar to the subjects' unimpaired breathing as measured by a stop watch during unobtrusive observation. Second, the difference in the effects caused by the two systems was not due to the use of a nasal breathing route while wearing a mask, versus an enforced oral route when a nose-clip was worn with the mouthpiece. When a nose-clip was worn under a mask, the ensuing ventilation did not differ significantly from that measured when the mask alone was worn.

A possible explanation of the different results obtained by Askanazi et al (1980) and Hirsch \& Bishop (1982) relates to the issue of dead-space. Hirsch \& Bishop (1982) matched the dead-space of the two systems in each subject, Askanzi et al. (1980), however, had a larger dead-space in the mask condition. This increased dead-space would have provided a greater load to breathing and thus required a greater minute ventilation to provide the same level of alveolar ventilation.

In conclusion it would appear that wearing a mask or a mouthpiece and nose-clip is associated with increased tidal volume and in most investigations with increased level of minute ventilation. The cause of these effects is uncertain and may be due to either stimulation of the nasal mucosa, stimulation of the oral region, increasing dead space, or altering the normal route of air flow. Wearing a mask is also associated with increased levels of ventilation, although it is possible that the effect with this apparatus is due to dead space. 


\subsection{Implications for Awake - Asleep Comparisons.}

All the above studies reported the effects of measuring apparatus on the ventilation of awake subjects. These effects have been ascribed to three causes: increased dead-space and thus increased ventilatory load; increased sensory stimulation; and psychological factors related to awareness of the equipment. Psychological factors, while being difficult to measure, are of obvious importance if ventilation is to be compared at different levels of wakefulness. Dead-space or stimulation of the facial, oral, or nasal regions presumably remains constant, whether the subject is awake or asleep (Honda, Hayashi, Yoshida, Ohyabu, Nisibayashi \& Kimura, 1983). However, any influence on ventilation due to awareness of the equipment, will cease with the attainment of sleep. Thus, there is the possibility of confounding the effects of equipment with those of wakefulness per se. As behavioural measures of awareness, such as reaction time, have displayed significant decreases from wakefulness to stage one sleep (Ogilvie \& Wilkinson, 1984), there is evidence to suggest that awareness related equipment effects may cease early in the sleep onset period.

The majority of studies reporting ventilation as being lower during NREM sleep have used a mask, or mouthpiece (Birchfield et al., 1959; Bulow, 1963; Bulow \& Ingvar, 1961; Douglas et al., 1982b; Douglas et al., 1982a; Gleeson et al., 1986; Gothe et al., 1986; Hudgel et al., 1984; Reed \& Kellogg, 1958; 1960a; 1960b; Robin et al., 1958; Skatrud \& Berssenbrugge, 1983 ; Skatrud \& Dempsey, 1983; White, 1986; White et al., 1982; White et al., 1985b; and Yamashiro et al., 1987 ). However, there have been reports of ventilation decreasing with sleep when neither a mask nor a mouthpiece was used (Lugaresi et al., 1978; Rist et al., 1986; Shore et al., 1985; Tabachnik et al., 1981; and Gothe et al., 1981). In these studies, some form of magnetometer or plethysmograph provided the ventilation measurement 
While these last studies provide indirect evidence that awake-to-sleep changes in ventilation occur whether or not a mask is worn, a detailed evaluation of the effects of such apparatus on ventilation during sleep is needed. The following chapter presents the results of a study designed to evaluate the effect of the mask apparatus used in the two previous studies of the present thesis. 
CHAPTER EIGHT

EQUIPMENT ARTIFACT AND VENTILATION DURING SLEEP ONSET 


\section{CHAPTER EIGHT}

\section{EQUIPMENT ARTIFACT AND VENTILATION \\ DURING SLEEP ONSET}

\subsection{Introduction}

As mentioned in the previous chapter, the use of a mask and breathing valve has been found to influence the level of ventilation measured in subjects while awake. If the effects are specific to wakefulness, the possibility exists that the sleep related decrease in ventilation reported in the two previous studies may have been influenced in some way by the mask and valve apparatus used in them. The aim of the present experiment was to assess whether or not a decrease in ventilation occurred in association with the appearance of theta EEG activity when ventilation was measured by abdominal and thoracic respiratory movement. The prediction based on the wakefulness drive hypothesis was that the sleep related decrease in ventilation would occur independently of the device used to measure it.

\subsection{Method}

\subsubsection{Subjects and Design}

Two young male subjects (aged $21 \& 22$ ) who were free from respiratory pathology and were normal sleepers were used in the study. Both subjects had participated in earlier experiments in the laboratory and were used to the equipment and the laboratory environment. Each subject spent an adaptation night in the laboratory followed by nine experimental nights, three nights in each of the three conditions. In condition MASK the subjects wore a mask and abdominal and thoracic strain gauges. This condition replicated experiments one and two. In condition CATH, subjects had a nasal catheter attached immediately below the nares. In addition subjects wore the strain gauges. This condition eliminated equipment dead space and minimised facial stimulation, but still 
allowed end-tidal $\mathrm{CO}_{2}$ and $\mathrm{O}_{2}$ values to be measured. In the GAUGE condition, subjects wore the strain gauges alone. Each subject had a different order of conditions based on a modified latin squares design. The orders were as follows, subject D.L.: M, C, G, C, M, G, M, G, C; and subject A.C.: C, G, M, G, M, C, G, C, M.

The event of interest in the study was sleep onset and consequently a number of sleep onsets were recorded on each night. Subjects were put to bed close to their normal bed time and instructed to stay awake for ten to fifteen minutes, they were then left in a darkened bedroom with minimal auditory or visual stimulation. After sleep (stage 2) had been achieved and maintained for at least ten minutes, the subjects were reawakwened. They remained in bed reading, talking and listening to the radio until they reported feeling sufficiently awake to continue, at which time the procedure was repeated. The heights and weights of the subjects were as follows: A.C. $186 \mathrm{~cm}$. and $85 \mathrm{Kg}$; D.L. $196 \mathrm{~cm}$. and $83 \mathrm{Kg}$.

\subsubsection{Equipment}

In all three conditions subjects wore two $20 \mathrm{~cm}$. mercury in rubber strain gauges one at nipple level and the other at the level of the umbilicus. Each gauge was taped the subject's back and passed through two $3 \mathrm{~cm}$. silicone rubber loops which enabled it to be secured to their front without the movement of the gauge being impeded. The subjects wore a Respitrace $®$ retainer over their trunk region, which further aided the stability of the gauges without inhibiting their mobility. The retainer was taped in place at both shoulders and to the lower back and abdomen. The gauges were attached at one end to a length of thin cord in which a series of loops were fixed at five centimeter intervals. This enabled the gauges to be measured within the same interval of their total tension on each night. This was necessary to diminish the problem of the lack of linearity in response that gauges exhibit across the total range of potential tension. The strain gauges were the only respiratory related equipment used in the GAUGE condition. 
In addition to the above, in the MASK condition the equipment used was identical to that of the two previous experiments, with the addition of an Applied Electrochemistry CD-3A Carbon Dioxide Analyser. Subjects wore an anesthetic mask fitted with an inflatable cuff to minimize leakeage and held in place with a head strap. The mask was attached to a three way breathing valve (Rudolf \#2600). The valve dead space was 33 $\mathrm{ml}$. with the total dead space of mask and valve being $113 \mathrm{ml}$. for A.C. and $118 \mathrm{ml}$. for D.L. due to the subjects having slightly different facial configurations.

In the CATH condition subjects wore the strain gauges as described above with end tidal oxygen and carbon dioxide being monitored via a nasal catheter. The catheter used was a standard oxygen administration hospital catheter which passed under the nose and was held in place by two small outlets which sat approximately four millimeters inside the two nostrils. Outlets from the catheter led to the oxygen and carbon dioxide analyser. The device was very light and unobtrusive and after initial administration, subjects reported that they were unaware of it being worn.

\subsubsection{Variables}

The respiratory variables investigated in the MASK condition were: respiratory cycle duration (CD); the volume of air expired (Vt), corrected to S.T.P. (saturated); fractional end tidal levels of $\mathrm{O}_{2}\left(\mathrm{FetO}_{2}\right)$, and $\mathrm{CO}_{2}\left(\mathrm{FetCO}_{2}\right)$; minute ventilation (VE); minute $\mathrm{O}_{2}$ consumption $\left(\mathrm{VO}_{2}\right)$; minute $\mathrm{CO}_{2}$ production $\left(\mathrm{VCO}_{2}\right)$; and minute estimation of $\mathrm{VE}$ extrapolated from individual breath values (VE(Extrap)).

In the CATH condition the variables were: $\mathrm{CD} ; \mathrm{Vt}(\mathrm{Regr}) \mathrm{FetO}_{2}$; $\mathrm{FetCO}_{2}$; and VE(Extrap)*, and in the GAUGE condition: CD; Vt(Regr); and VE(Extrap)*. The variable $\mathrm{Vt}(\mathrm{Regr})$ was the estimate of $\mathrm{Vt}$ calculated from the regression equations between thoracic and abdominal strain gauge amplitudes and $\mathrm{Vt}$ in the MASK condition. $\mathrm{VE}($ Extrap)*, was an estimate of minute ventilation, and was calculated by cross 
multiplying the respiratory frequency $(60 / \mathrm{CD})$ by the regressed tidal volume (Vt Regr). $\mathrm{CD}$ was taken from the abdominal measurement as it was difficult to estimate $\mathrm{CD}$ from the thoracic respiratory movements.

Sleep state was ascertained for consecutive one minute epochs for the EEG, EOG and EMG recordings by an experienced scorer using the standard criteria (Rechtshaffen \& Kales, 1968). in addition during particular portions of each sleep onset, the EEG associated with each breath was classified into three categories according to the dominant frequency band: alpha or beta (wake); theta (stage 1) or theta in association with $\mathrm{K}$ complexes or spindles (stage 2).

\subsection{Results}

\subsubsection{Multiple Regression Analysis}

A multiple regression analysis was performed for each subject to ascertain the best method of predicting tidal volume from the abdominal and thoracic strain gauge measurements. This analysis involved all breaths from the MASK condition in which tidal volume, and abdominal and thoracic amplitudes could be measured, with those associated with alpha EEG being analysed separately to those with theta or stage two. Unfortunately, neither subject was able to complete all sessions of the experiment with only one set of strain gauges. Both required one gauge to be replaced due to breakage. This factor resulted in two sets of regression analyses for each subject, one for each of their strain gauge combinations.

The variables entered into the multiple regression analysis were: abdominal amplitude (A); thoracic amplitude (T); abdominal and thoracic amplitudes added together $(\mathrm{A}+\mathrm{T})$; abdominal $\times 2$ added to thoracic $(2 \mathrm{~A}+\mathrm{T})$; thoracic $\times 2$ added to abdominal $(2 \mathrm{~T}+\mathrm{A})$; and finally a ratio of the two measurements calculated by $((\mathrm{T}-\mathrm{A}) /((\mathrm{T}+\mathrm{A}) / 2))$ +1 (Naifeh \& Kamiya, 1981), (ATR). The regression equations resulting from these 
analyses are presented in table 8.1. In each case the multiple regression produced an equation which was based on a single variable, however the particular variable differed between analyses.

The regression equations which were selected differed from previous studies in two important respects. The first was that the equations were not the same for wakefulness and sleep. This result is sensible in terms of reports from several studies which have indicated that thoracic and abdominal contributions to ventilation vary between wakefulness and sleep. That is, supine subjects have relatively more abdominal respiratory movement when awake than asleep. (Goldie \& Green, 1961; Gothe et al., 1981; Gothe et al., 1982; Mortola \& Anch, 1978; Naifeh \& Kamiya, 1981; Tabachnik et al., 1981; and Timmons et al., 1977). In previous studies estimates of Vt from abdominal and chest expansions have been calculated from awake data and applied to both awake and sleeping conditions (Gothe et al., 1981; Rist et al., 1986; Shore et al., 1985; and Tabachnik et al., 1981 ).

Varying the strain gauge combinations used between sessions also required the calculation of different regression equations. This was despite the same abdominal strain gauge being used in each combination and all thoracic gauges being of the same make and length. In addition, care was taken to place the gauges in the same positions in each session. Thus a particular regression equation, calculated in one state, cannot be used to explain data across states or in different sessions if the equipment is varied between them. To increase accuracy it would appear necessary to conduct a MASK condition sleep onset for each experimental session. Only in this manner would it be possible to guarantee equality between the data used for estimation purposes and that on which the estimation was based. 
The second result was that the correlation coefficients reported in table 8.1 are lower than those previously reported in the literature. Previous studies which have used thoracic and abdominal movement to estimate ventilation during sleep, have all based their estimations on calibration procedures conducted while the subject was awake (Gothe et al., 1981; Rist et al., 1986; Shore et al., 1985; and Tabachnik et al., 1981). Typically the calibration procedure has involved breathing into a spirometer for a short period of time, while wearing the abdominal and thoracic transducers (Gothe et al., 1981, 8 - 10 breaths; Rist et al., 1986, 80 seconds; and Tabachnik et al., 1981, 30 breaths). These procedures have typically produced regression coefficients of 0.8 or better, due to the controlled nature in which the data was collected, often involving manipulation of breathing over a large range of levels. The results of the present experiment thus indicate that the previous studies have overestimated the accuracy of their procedures for estimating ventilation from abdominal and thoracic movements. 


\section{Table 8.1}

Regression Equations Relating Abdominal and Thoracic Movement to Tidal Volume

\begin{tabular}{|c|c|c|c|c|}
\hline State & Subject & Equation & $r$ value & significance \\
\hline \multirow[t]{4}{*}{ Wake } & D.L. (1) & $179.05+2.26 \times(2 \mathrm{~T}+\mathrm{A})$ & 0.70 & $\mathrm{p}<.001$ \\
\hline & D.L. (2) & $6.60+4.47 \times(\mathrm{A}+\mathrm{T})$ & 0.72 & $\mathrm{p}<.001$ \\
\hline & A.C. (1) & $72.09+2.42 \times(2 \mathrm{~T}+\mathrm{A})$ & 0.51 & $\mathrm{p}<.001$ \\
\hline & A.C. (2) & $87.83+2.17 \times(2 \mathrm{~T}+\mathrm{A})$ & 0.60 & $\mathrm{p}<.001$ \\
\hline \multirow[t]{4}{*}{ Sleep } & D.L. (1) & $140.41+2.48 \times(2 \mathrm{~A}+\mathrm{T})$ & 0.62 & $\mathrm{p}<.001$ \\
\hline & D.L. (2) & $25.61+2.73 \times(2 \mathrm{~A}+\mathrm{T})$ & 0.75 & $\mathrm{p}<.001$ \\
\hline & A.C. (1) & $200.96+2.14 \times(\mathrm{A})$ & 0.44 & $\mathrm{p}<.001$ \\
\hline & A.C. (2) & $-7.34+3.77 \times(\mathrm{A}+\mathrm{T})$ & 0.69 & $\mathrm{p}<.001$ \\
\hline
\end{tabular}

Regression equations describing the relationship between combinations of abdominal and thoracic respiratory movements with tidal volume. (1) and (2) refer to two different combinations of strain gauges used on different nights. A refers to abdominal amplitude, and $\mathrm{T}$ to thoracic. 


\subsubsection{Ventilation During Sleep Onset}

As the aim of the present experiment was to determine the effects of wearing the mask on the changes to ventilation at sleep onset, only two of the five analysis techniques used in the previous studies were required. These were method one, describing the pattern of change in minute values from stable wakefulness to stage two sleep, and method four describing the pattern of change in breath values around the point of theta onset.

\section{Method One: Minute values from wakefulness to stage 2 sleep.}

The initial analysis of the data summarised the changes in $\mathrm{VE}, \mathrm{VO}_{2}$, and $\mathrm{FetO}_{2}$ over the total sleep onset period (see table 8.2). The results of this analysis for the MASK condition demonstrated reductions in VE in each subject from wake to stage 2 sleep (see table 8.2), with the decrease occurring in $100 \%$ of transitions in both subjects. The percentage fall was similar in both subjects. D.L. displayed a $25 \%$ decrease (1.76 litres/min) and A.C. a $23 \%$ decrease (1.52 litres/min). In both subjects a substantial proportion of the decrease was evidenced within the first minute of the transition period (D.L., 50\%; A.C., 75\%). Both subjects in the MASK condition also demonstrated decreases in metabolic rate as measured by both $\mathrm{VO}_{2}$ and $\mathrm{VCO}_{2}$. In both subjects the decrease in metabolic rate was associated with a decrease in $\mathrm{FetO}_{2}$ and an increase in $\mathrm{FetCO}_{2}$. D.L. displayed a $5 \%$ decrease in $\mathrm{FetO}_{2}\left(0.74 \% \mathrm{O}_{2}\right)$ and an $8 \%$ increase in FetCO $2\left(0.48 \% \mathrm{CO}_{2}\right)$. Similarly, A.C. displayed a $5 \%$ decrease in $\mathrm{FetO}_{2}\left(0.72 \% \mathrm{O}_{2}\right)$ and an \% increase in $\mathrm{FetCO}_{2}\left(0.46 \% \mathrm{CO}_{2}\right)$. 
For the analysis of the CATH condition, ventilation is only presented during the stable awake and sleep periods for the CATH and GAUGE conditions. The transition periods involved breaths associated with both alpha and theta activity and thus neither the awake or asleep regression equations were appropriate for all breaths within a minute, or all minutes within a transition quartile. The regressed ventilation measures from the CATH condition demonstrated decreases from wakefulness to sleep in every transition. In both subjects, the magnitude of the decreases was smaller than that demonstrated in the MASK condition. D.L. showed a $21 \%$ decrease (1.48 litres/min) and A.C. a $20 \%$ decrease (1.05 litres/min). The CATH condition was also associated with variations in the end-tidal measurements of $\mathrm{O}_{2}$ and $\mathrm{CO}_{2}$ in every sleep onset, which were similar to those which occurred in the MASK condition in both subjects (see figure 8.1). D.L. displayed a $5 \%$ decrease in $\mathrm{FetO}_{2}\left(0.79 \% \mathrm{O}_{2}\right)$ and a $7 \%$ increase in FetCO $2\left(0.44 \% \mathrm{CO}_{2}\right)$. A.C.displayed a $5 \%$ decrease in $\mathrm{FetO}_{2}\left(0.73 \% \mathrm{O}_{2}\right)$ and a $6 \%$ increase in $\mathrm{FetCO}_{2}\left(0.37 \% \mathrm{CO}_{2}\right)$.

The analysis of $\operatorname{VE}($ Regr) from the GAUGE condition indicated that ventilation decreased in every sleep onset for both subjects. The $18 \%$ decrease $(1.04 \mathrm{litres} / \mathrm{min})$ of subject D.L. was slightly less than that of the other two conditions, whereas the $29 \%$ decrease of A.C. (1.73 litres/min) was slightly higher than that of the MASK and CATH conditions.

Two factor (sleep state by experimental condition) analyses of variance were conducted using VE(Regr) for each subject (see table 8.4). For both subjects, there was a significant effect of experimental condition displayed for ventilation, with the MASK condition producing higher levels than the other two conditions. The interaction between state and condition was significant for both subjects, indicating that the magnitude of the ventilation decrease from wakefulness to sleep was altered by the measurement equipment used. This result is later considered in detail in the discussion. 
Table 8.2

Respiration during sleep onset : Minute values for the MASK, CATH and GAUGE conditions.

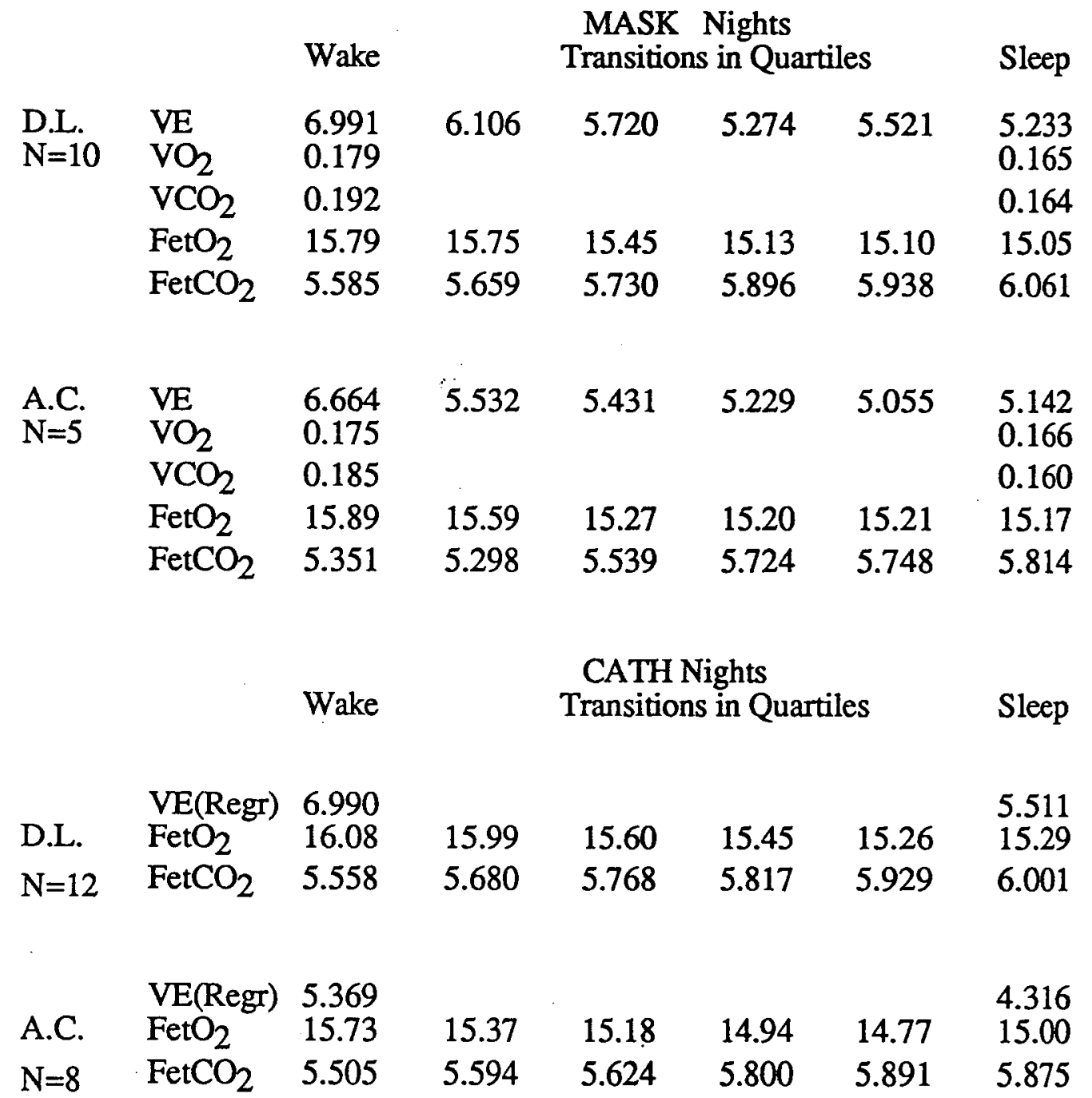

Wake

GAUGE Nights

Transitions in Quartiles Sleep

D.L. VE(Regr) 5.669

4.629

$\mathrm{N}=7$

A.C. $\quad$ VE(Regr) 5.976

$\mathrm{N}=3$

Changes in respiratory variables during the final 10 minutes of wake, the transitional period of mixed Wake/Stage 1 sleep and the first 10 minutes of Stage 2 sleep. The variable length transitional period has been divided into quartiles. The reported values are averages over sleep onsets where $N$ is the number of sleep onsets meeting appropriate criteria (see text). VE, VE(Regr) and $\mathrm{VO}_{2}$ are expressed in litres per minute and $\mathrm{FetO}_{2}$ in percentages. See glossary for explanation of abbreviations. 
Figure 8.1

A.C.

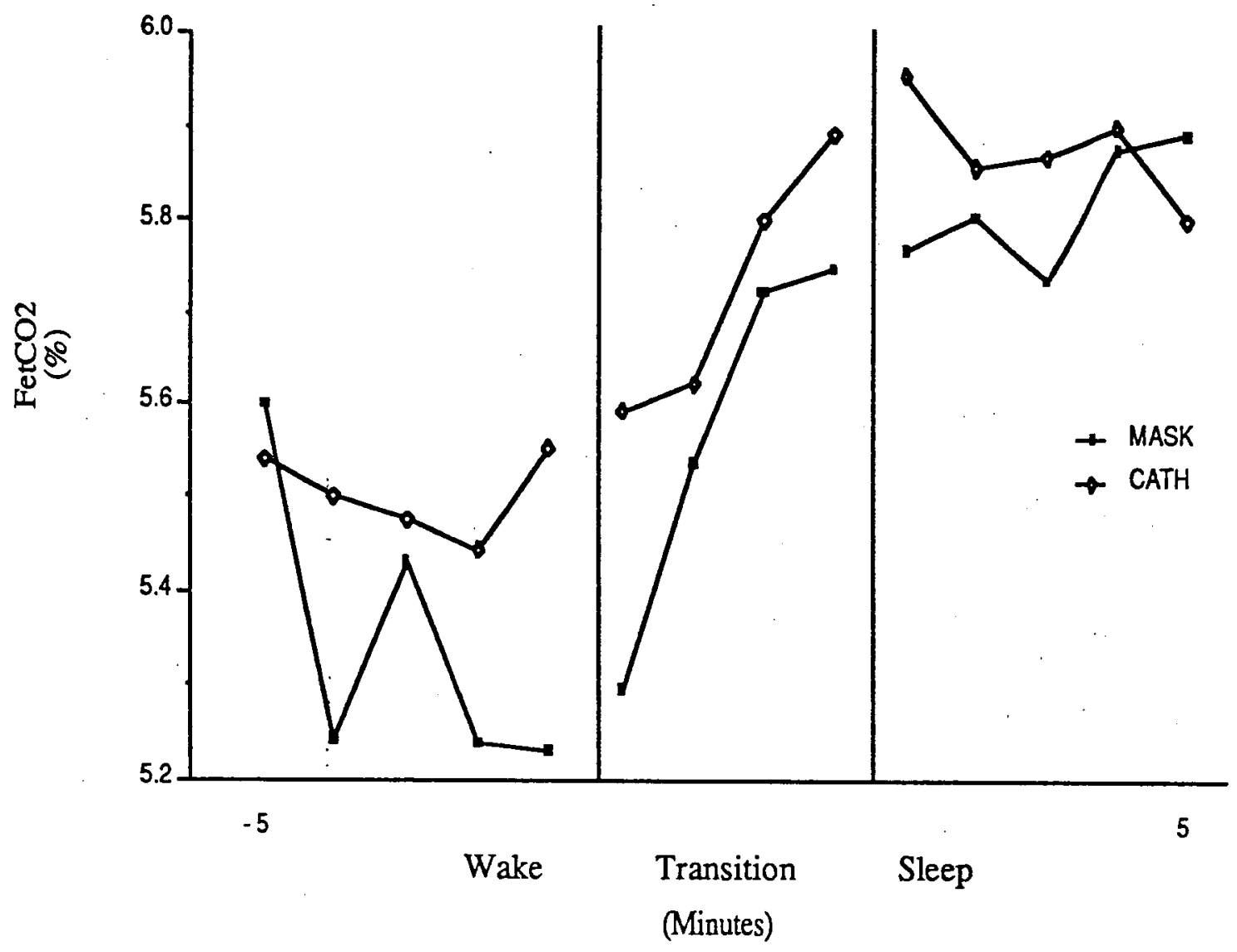

Figure 8.1 Minute values of end tidal $\mathrm{CO}_{2}$ for subject A.C. during the last five minutes of wake, the transitional period of mixed wake/stage 1 sleep, and the first five minutes of stage 2 sleep. Data is presented from the MASK and CATH conditions. 


\section{Method Four: Breath by breath values at awake-theta transitions}

In order to examine the relationship between ventilation and the cessation of alpha activity in more detail, smooth and relatively rapid transitions into stage 2 sleep were identified. The data analysed in each transition was the last five breaths before and the first 35 breaths following the onset of theta activity. The data was then averaged across transitions within subjects in a manner analogous to Averaged Evoked Responses. The analysis indicated that ventilation decreased in association with the onset of theta activity in all three conditions.

Subject D.L. demonstrated a decrease in VE (Extrap) of 16\% (0.991 litres/min) in the MASK condition. The decrease was mediated by a $14 \%$ increase in CD $(0.568$ secs.), with no change in $\mathrm{Vt}$. $\mathrm{FetO}_{2}$ decreased with theta onset (3\%) and $\mathrm{FetCO}_{2}$ demonstrated an increase (2\%). The changes in all variables with the exception of $\mathrm{Vt}$ were substantial within the first five breaths following theta onset. The same pattern of results was evidenced in the CATH condition. VE (Extrap)* decreased by $12 \%(0.779$ litres/min) with theta onset due to a $16 \%$ increase in $\mathrm{CD}(0.592 \mathrm{secs})$, FetO 2 decreased (4\%, see figure 8.2) and $\mathrm{FetCO}_{2}$ increased (5\%). Likewise in the GAUGE condition, VE (Extrap)* demonstrated a $13 \%$ decrease $(0.752$ litres $/ \mathrm{min})$ with the onset of theta activity due to a $15 \%$ increase in $\mathrm{CD}(0.550$ secs $)$. 
Table 8.3

Breath by breath changes in respiratory variables before and after the transition from EEG alpha/beta to theta activity in the MASK,

CATH and GAUGE conditions.

Subject

Alpha

MASK Nights

$\begin{array}{lllllll} & & & 1-5 & 6-10 & 11-20 & 21-35 \\ & \mathrm{VE}(\text { Extrap) } & 6.086 & 5.026 & 4.953 & 5.329 & 5.095 \\ & \mathrm{Vt} & 0.349 & 0.345 & 0.330 & 0.351 & 0.342 \\ \text { D.L. } & \mathrm{CD} & 3.478 & 4.128 & 4.018 & 3.975 & 4.046 \\ \mathrm{~N}=6 & \mathrm{FetO}_{2} & 15.56 & 15.32 & 15.19 & 15.32 & 15.04 \\ & \mathrm{FetCO}_{2} & 5.754 & 5.991 & 5.942 & 5.849 & 6.002 \\ & & & & & & \\ & \mathrm{VE}(\text { Extrap) } & .7 .158 & 5.469 & 5.112 & 5.412 & 5.352 \\ & \mathrm{Vt} & 0.368 & 0.311 & 0.294 & 0.312 & 0.307 \\ \mathrm{A.C} . & \mathrm{CD} & 3.097 & 3.475 & 3.511 & 3.517 & 3.506 \\ \mathrm{~N}=8 & \mathrm{FetO}_{2} & 15.67 & 15.48 & 15.29 & 15.30 & 15.16 \\ & \mathrm{FetCO}_{2} & 5.696 & 5.759 & 5.766 & 5.835 & 5.811\end{array}$

Subject

Alpha

CATH Nights

\begin{tabular}{lllllll} 
Subject & & Alpha & \multicolumn{4}{c}{ Breaths following cessation of alpha } \\
& & & $1-5$ & $6-10$ & $11-20$ & $21-35$ \\
& $\mathrm{VE}($ Extrap)** & 6.427 & 5.776 & 5.866 & 5.483 & 5.648 \\
& $\mathrm{Vt}($ Regr) & 0.339 & 0.346 & 0.368 & 0.350 & 0.353 \\
D.L. & $\mathrm{CD}$ & 3.159 & 3.585 & 3.717 & 3.830 & 3.751 \\
$\mathrm{~N}=6$ & $\mathrm{FetO}_{2}$ & 15.99 & 15.61 & 15.45 & 15.40 & 15.31 \\
& $\mathrm{FetCO}_{2}$ & 5.550 & 5.753 & 5.754 & 5.748 & 5.812 \\
& & & & & & \\
& $\mathrm{VE}($ Extrap)* & 5.603 & 3.850 & 4.004 & 4.197 & 4.247 \\
& $\mathrm{Vt}($ Regr $)$ & 0.257 & 0.192 & 0.195 & 0.205 & 0.213 \\
A.C. & $\mathrm{CD}$ & 2.752 & 2.992 & 2.922 & 2.931 & 3.009 \\
$\mathrm{~N}=7$ & $\mathrm{FetO} 2$ & 14.99 & 14.80 & 14.66 & 14.73 & 14.78 \\
& $\mathrm{FetCO} 2$ & 5.643 & 5.735 & 5.692 & 5.690 & 5.706
\end{tabular}


Table 8.3 (continued)

Subject

GAUGE Nights

\begin{tabular}{|c|c|c|c|c|c|c|}
\hline \multirow[t]{2}{*}{ Subject } & & \multirow[t]{2}{*}{ Alpha } & \multicolumn{4}{|c|}{ Breaths following cessation of alpha } \\
\hline & & & $1-5$ & $6-10$ & $11-20$ & $21-35$ \\
\hline & VE(Extrap)* & 5.779 & 5.003 & 4.967 & 4.764 & 5.027 \\
\hline D.L. & Vt (Regr) & 0.308 & 0.304 & 0.300 & 0.300 & 0.314 \\
\hline$N=6$ & $\mathrm{CD}$ & 3.198 & 3.646 & 3.624 & 3.778 & 3.748 \\
\hline & VE(Extrap)* & 5.660 & 3.239 & 3.407 & 3.762 & 3.97 \\
\hline A.C. & Vt (Regr) & 0.266 & 0.167 & 0.179 & 0.204 & 0.212 \\
\hline$N=5$ & $\mathrm{CD}$ & 2.820 & 3.094 & 3.152 & 3.253 & $3.2+>>>3$ \\
\hline
\end{tabular}

Breath by breath changes in respiratory variables during the transition from sustained alpha activity of at least one minute to sustained theta leading to Stage 2 sleep. The alpha values are averages over the last 5 breaths before the cessation of alpha while the remaining values are averages over varying intervals (see table) to a total of 35 breaths following the cessation of alpha activity. $\mathrm{N}=$ the number of transitions available for each subject. VE(Extrap) and VE(Extrap)* are expressed in litres/min, $\mathrm{Vt}$ and $\mathrm{Vt}(\mathrm{Regr})$ in litres, cycle duration (CD) in seconds and $\mathrm{FetO}_{2}$ in percentages. See glossary for explanation of abbreviations. 
Subject A.C. displayed a 25\% decrease in VE (Extrap) (1.806 litres/min., see figure 8.3), in the MASK condition, which was mediated by both a $17 \%$ decrease in Vt (61 ml.) and a $12 \%$ rise in CD (0.409 secs.). Theta onset was also associated with a decreased $\mathrm{FetO}_{2}(3 \%)$ and an increased $\mathrm{FetCO}_{2}(2 \%)$. The same pattern was evidenced in the CATH condition with a 24\% decrease VE (Extrap)* (1.356 litres/min), a 17\% decrease in $\mathrm{Vt}(\mathrm{Regr})(44 \mathrm{ml}$.) and a $9 \%$ increase in $\mathrm{CD}(0.257$ secs.) Further there were slight changes in $\mathrm{FetO}_{2}(2 \%)$ and $\mathrm{FetCO}_{2}(1 \%)$. The GAUGE condition displayed a 30\% decrease VE (Extrap)* (1.689 litres/min), a 20\% decrease in Vt (Regr) (54 ml.), and a $12 \%$ increase in $\mathrm{CD}(0.383 \mathrm{sec}$.) associated with theta onset. In all three conditions, the changes in all variables were substantial within the first five theta breaths.

Two factor (sleep state by experimental condition) analyses of variance were conducted using VE(Extrap)* for each subject. The results of these analyses are presented in Table 8.4. For both subjects, there was a significant effect of experimental condition displayed for ventilation, with the MASK condition producing higher levels than the other two conditions. The most important result from the analyses was that the interaction between state (alpha, theta) and condition (MASK, CATH, GAUGE) was not significant for either subject. Thus the magnitude of the ventilation decrease from alpha to theta activity was not systematically altered by the measurement equipment used. 


\section{Table 8.4}

Summary ANOVA tables for the analysis of equipment condition

\begin{tabular}{|c|c|c|c|c|c|}
\hline Subject & Variable & Effect & $\mathrm{F}$ & $\mathrm{p}$ & Degrees of Freedom \\
\hline \multirow[t]{6}{*}{ D.L. } & VE(Extrap)* & state & 127.97 & 0.000 & 1,277 \\
\hline & & condition & 34.47 & 0.000 & \\
\hline & & $\mathrm{s} \times \mathrm{c}$ & 0.726 & 0.485 & \\
\hline & VE(Regr) & state & 48.83 & 0.000 & 1,237 \\
\hline & & condition & 59.97 & 0.000 & \\
\hline & & $\mathrm{s} \times \mathrm{c}$ & 41.26 & 0.000 & \\
\hline \multirow[t]{6}{*}{ A.C. } & VE(Extrap)* & state & 112.52 & 0.000 & 1,285 \\
\hline & & condition & 13.31 & 0.000 & \\
\hline & & $\mathrm{sxc}$ & 0.364 & 0.695 & \\
\hline & VE(Regr) & state & 132.73 & 0.000 & 1,135 \\
\hline & & condition & 21.70 & 0.000 & \\
\hline & & $\mathrm{s} \times \mathrm{c}$ & 4.148 & 0.018 & \\
\hline
\end{tabular}

Analysis of variance of the two factors: state (alpha, theta); and experimental condition (MASK, CATH, GAUGE). VE(Extrap)* was calculated using breath values from transitions identified using the rules of the fourth analysis method. The alpha values were the last five breaths preceding the onset of theta activity. The theta values were breaths 26 to 35 following theta onset. $\mathrm{VE}$ (Regr) was calculated using minute values from transitions identified using the rules of the first analysis method. The wake values were the last five minutes preceding theta onset. The sleep values were the first five minutes following the onset of stage 2 sleep. See glossary for explanation of abbreviations. 
Figure 8.2

A.C.
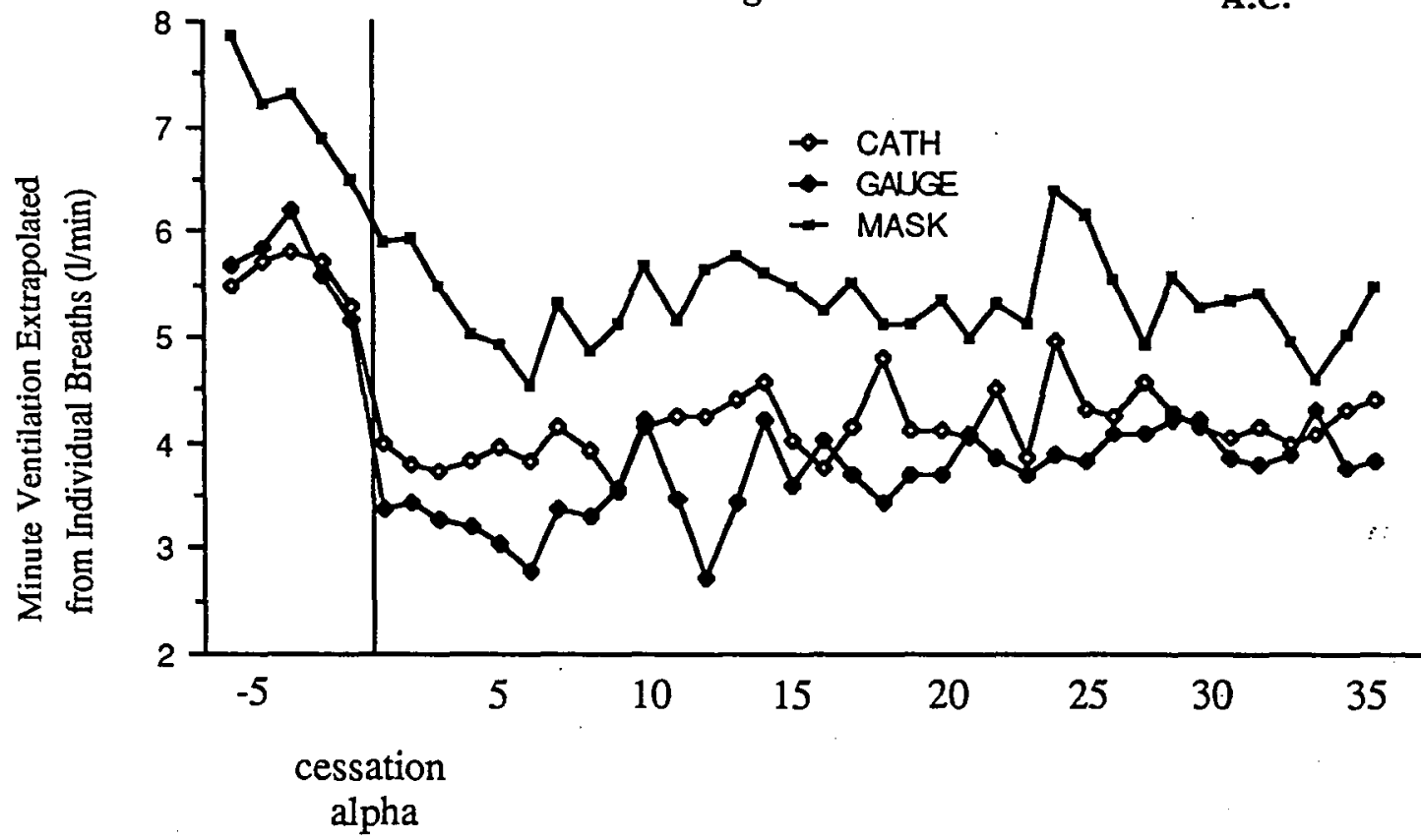

Breaths before and after cessation of alpha

Figure 8.2 Minute ventilation extrapolated from individual breaths for subject A.C. The values for the final 5 breaths before cessation of alpha in the EEG, and the following 35 breaths are shown. Data is presented from the MASK, CATH, and GAUGE conditions. 


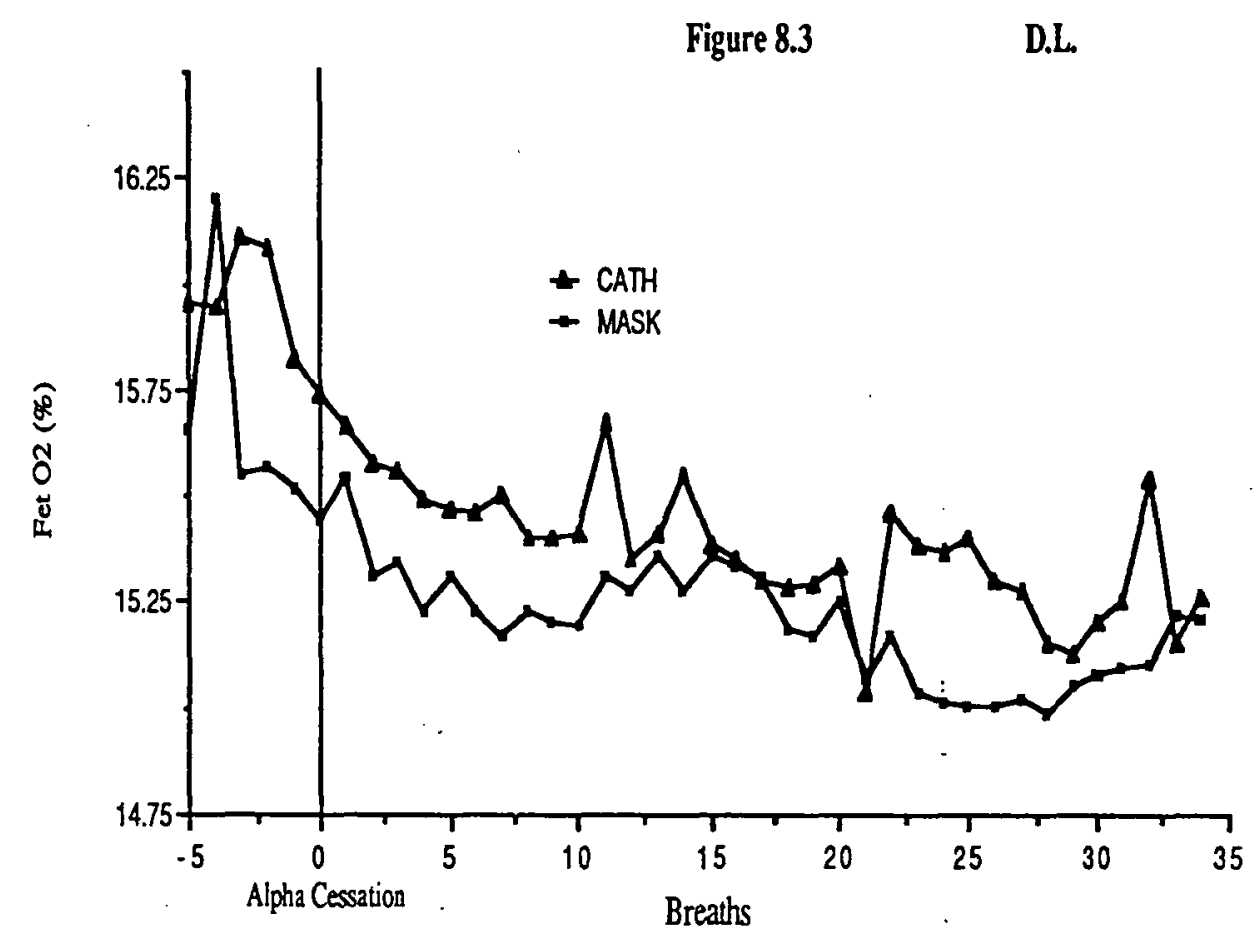

Figure 8.3 End tidal Oxygen for subject D.L. The values of the final 5 breaths before cessation of alpha in EEG, and the following 35 breaths are shown. Data is presented from the CATH and MASK conditions. 


\subsection{Discussion}

The analysis conducted using minute values indicated that the magnitude of the sleep related decrease varied significantly between conditions (see table 8.4). There are however three points which can be made regarding this result. Firstly, the ordering of the relative sizes of the change across conditions varied between the two subjects. Thus in subject D.L. the greatest decrease occurred in the MASK condition, while in subject A.C. the greatest change occurred in the GAUGE condition. Secondly, despite the differing magnitudes, substantial changes occurred with sleep in all conditions for each subject. Thirdly, the analysis conducted using breath values (see table 8.4 ) indicated that the pattern of change in ventilation from alpha/beta to theta activity was unaffected by the use of a mask and breathing valve. Thus while the overall level of ventilation appeared to be increased by the mask in both subjects as has been reported in previous studies (Askanazi, 1980; and Hirsch \& Bishop, 1982), the decrease in ventilation at theta onset reported in the first two experiments of the present thesis (chapters 4 and 6) was not due to the use of a mask and breathing valve.

The results indicate that separate regression equations are required to describe the relationship between respiratory movement and tidal volume in wakefulness and sleep. This is probably due to the different relative contributions made to ventilation by the rib cage and diaphragm during sleep in supine subjects (Goldie \& Green, 1961; Gothe et al., 1981; Gothe et al., 1982; Mortola \& Anch, 1978; Naifeh \& Kamiya, 1981; Tabachnik et al., 1981; and Timmons et al., 1977). Previous studies which have attempted to predict ventilation from respiratory movements have used a calibration procedure based on a small number of breaths of varying volumes while the subject is awake (Gothe et al., 1981; Rist et al., 1986; Shore et al., 1985; and Tabachnik et al., 1981). The data presented in the present experiment would indicate that this practice could potentially lead to error when ventilation was investigated in sleeping subjects, as 
the relationship between respiratory movement and ventilation varies between wakefulness and sleep. Further, it is likely that the regression coefficients reported in the previous studies, have overestimated the strength of the relationship in normal breathing, due to the artificial nature of the calibration data collection.

The present study also found that varying the strain gauges used to measure thoracic movement results in different regression equations being required to describe the data. The combination of these last two points indicates that much more care needs to be taken in collecting data on which regression equations can be calculated than was previously thought to be the case. In order to accurately estimate tidal volume during sleep, it would be necessary to measure abdominal and thoracic movement with and without some direct measure of tidal volume during sleep in the same session, with the indirect measuring equipment not varying between the two measurements.

The results of the study reinforce those of the two earlier experiments of the present thesis in indicating that theta onset is the point at which breathing consistently changes when subjects go to sleep. Further it indicates that the mask and valve apparatus used in those experiments was not a contributing factor to the effect. Thus the evidence for ventilation decreasing in association with cessation of a wakefulness drive is strengthened. 


\section{CHAPTER NINE}

DISCUSSION AND CONCLUSIONS 


\section{CHAPTER NINE DISCUSSION AND CONCLUSIONS}

The central emphasis of the three experiments of the present thesis was a description of the changes in ventilation which occur during the sleep onset period. These changes were interpreted in terms of the wakefulness drive hypothesis which predicts that ventilatory changes should be rapid, and consistently related to changes in brain activity related to onset of sleep. Both of these predictions were supported by the data. In all three experiments, ventilation decreased rapidly at the point of the onset of theta EEG activity, in almost all sleep onsets for all subjects.

\subsection{Male Subjects}

In the first experiment, the loss of alpha activity in the EEG during sleep onset was closely associated with a substantial, rapid, and highly predictable reduction in ventilation. While oxygen consumption also fell over the total sleep onset period (wake to stage 2 sleep), the consistent reduction in $\mathrm{FetO}_{2}$ indicated that the ventilation decrease was larger than that required by the diminished metabolic rate. It was speculated that the non-metabolic component in the ventilation decrease reflected the loss of wakefulness drive. The reduction in ventilation at sleep onset was consistent with informal observations reported in earlier papers (Bulow, 1963; and Naifeh \& Kamiya, 1981), though the experiment presented in chapter four was the first to accurately quantify the magnitude and temporal properties of the phenomenon. 


\subsection{Female Subjects}

There appear to be a number of differences between female and male subjects in terms of waking respiration, chemical control mechanisms during sleep, and the incidence of sleep related respiratory dysfunction. It has often been hypothesised that these differences reflect the fact that females have higher levels of circulating progesterone, especially during the luteal phase of the menstrual cycle. More recently, it has been hypothesised that sex differences might also be in part due to differential levels of testosterone, which appear to enhance the ventilatory sensitivity to hypoxia and hypercapnia.

The alteration of central respiratory drive by hormones and possible sex-differences in hypoxic and hypercapnic ventilatory responses, raised the question as to whether or not females exhibit changes in respiration at sleep onset. If females did not possess a wakefulness drive, it was predicted that the ventilation decrease with sleep would not demonstrate the rapidity and close association to changes in the EEG found in males. Further, there was a question as to whether different patterns of change were apparent during the high and low progesterone phases of the menstrual cycle, due to the possibility of an interaction between a wakefulness drive and the effects of progesterone.

The data from five female subjects in chapter six, demonstrated that the decrease in ventilation during sleep onset occurred rapidly at the point of theta onset. The ventilation decrease was shown to be greater than that required by decreasing metabolic rate as end tidal oxygen levels rose and the ratio of ventilation to metabolic rate fell with sleep in all subjects. The data were consistent with those reported for the male subjects of the first experiment.

There was some suggestion that male and females differed with respect to the timing and magnitude of the ventilatory change. These results must be treated with caution as the comparison of the magnitude and timing of respiratory changes at sleep onset were made on samples collected at different times and with slight differences in mehodology. 
The differences in the timing of the fall in ventilation is also compromised by the use of running aveages to smooth the ventilatory data and by the relative difficulty in identifying alpha-theta transitions in the female subjects. Nevertheless the data suggest the possibility that the timing of the changes in ventilatory control and brain activity may be slightly different in females, as opposed to males.

The differences in the magnitude of the ventilatory fall at the onset of theta activity revealed in the breath by breath analysis is perhaps of greater interest. The data suggest that either the magnitude of the process affecting the change in ventilation is different, or that male subjects are affected by an additional process. For example, in male subjects, in addition to a loss of wakefulness drive to ventilation, sleep onset may be associated with an increase in airway resistance, whereas females may only show the decrease in wakefulness drive.

The critical finding from this study is that females, like males, have large and rapid reductions in ventilation at sleep onset which are systematically associated with the loss of alpha activity and the appearance of theta in the EEG. As for males it is proposed that this change in part reflects the loss of a wakefulness drive to respiration during sleep.

Any statements regarding the effect of menstrual phase are limited because menstrual phase could be accurately identified in only two subjects. However, with these two, the analysis of the interaction between state (wake and sleep) and menstrual phase (luteal and follicular) indicated that the magnitude of the decrease in ventilation associated with sleep did not differ between the two phases. The pattern of the ventilation change was also found not to differ between the two phases. Thus, on the limited data available, menstrual phase, and therefore the concentration of progesterone, did not appear to interact in any way with the loss of a wakefulness drive. 


\subsection{The Effect of a Mask on Ventilation}

The results of the first two experiments were compelling. There were, however, a number of factors which suggested caution in the interpretation of the data. The reduction in ventilation attributable to a non-metabolic factor may have been a consequence of a variable, or variables, other than the loss of waking neural drive to ventilation, the most obvious being the use of a mask with a large mask and valve dead space.

Several studies have reported the effects of measurement apparatus on the ventilation of awake subjects. These effects have been ascribed to three causes: increased dead-space and thus increased ventilatory load; increased sensory stimulation; and psychological factors related to awareness of the equipment. Increased respiratory load and sensory stimulation will continue to influence data during sleep, in much the same way as they did during wakefulness. Thus any artifact produced should not be systematically related to the point of theta onset. However, any influence on ventilation, produced by awareness of the equipment, will cease with the acquisition of sleep. Further, it has been demonstrated that, behavioural measures of awareness, such as reaction time, display significant decreases from wakefulness to stage one sleep (Ogilvie \& Wilkinson, 1984). There is therefore evidence to suggest that awareness related equipment effects may cease early in the sleep onset period, and thus confound any changes provided by the cessation of a wakefulness drive.

The results presented in chapter eight indicated that the pattern of change in ventilation during sleep onset remained the same, whether or not a mask and breathing valve were used to measure ventilation. That is, when the measurement of ventilation was conducted using abdominal and thoracic strain gauges alone, ventilation levels still decreased rapidly at the point of theta onset and were associated with decreased $\mathrm{FetO}_{2}$ and increased $\mathrm{FetCO}_{2}$. Further, the magnitude of the theta related ventilation decrease was not affected by wearing a mask, as indicated by the absence of a significant 
interaction between the different data collection methods and awareness state (alpha/beta, theta).

Interestingly, different regression equations were required to describe the relationship between respiratory movement and tidal volume in wakefulness and sleep. This difference in procedure was probably caused by the change in the relative contributions of the abdomen and thorax to breathing during sleep and has not been considered in earlier studies (Gothe et al., 1981; Rist et al., 1986; Tabachnik et al., 1981). Different equations were also required when the equipment was varied between sessions. The results emphasised that a single regression equation can not be generalised across awareness states or experimental sessions without loss of accuracy.

\subsection{Implications for Awake-sleep mechanisms}

It has been hypothesised that during wakefulness, respiration is under metabolic and behavioural control, the latter being composed of both voluntary and state-related elements. There is much experimental evidence (see chapter three) to indicate that during NREM sleep, behavioural control is absent and respiration is solely determined by metabolic mechanisms. Further, during sleep, these mechanisms display an alteration of the set point which relates ventilation to the partial pressure of carbon dioxide, as well as a diminished responsiveness to the stimuli of hypoxia and hypercapnia. (Phillipson \& Bowes, 1986). This evidence is provided largely by the common finding that during sleep, hyperventilation leads to apnea whereas during wakefulness, "apnea is prevented over a wide range of alkalosis because some contribution from the awake state to inspiratory drive is sufficient to replace most of that provided by reduced $\mathrm{H}+$ " (Dempsey \& Skatrud, 1986).

As was cited in chapter three, wakefulness is thought to be maintained by cortical excitation resulting from activity within the ascending activity of the brain stem. (Remmers, 1981; Phillipson, 1978). While the onset of sleep is associated with a 
decrease in ascending reticular activity, it is uncertain as to whether this results from a passive withdrawal of activity or an active inhibition of the arousal system (Phillipson \& Bowes, 1986) the latter possibly mediated by forebrain mechanisms (Phillipson, 1978).

It is generally accepted that a wakefulness drive to respiration is also mediated by the brain stem reticular system. In a major review article, Phillipson \& Bowes (1986) state that "it is the progressive withdrawal of this neural ("non-cemical") stimulus through stages 1-4 of NREM sleep, because of the progressive reticular deactivation or inhibition, that results in a passive reduction of medullary respiratory neuronal activity". The notion of a progressive withdrawal of a wakefulness stimulus as presented by the above authors is not supported by the data presented in chapters four, six and eight of the thesis. Rather, the rapidity and magnitude of the ventilation decrease associated with variations in cortical arousal, indicate that the decrease of the wakefulness drive occurs rapidly rather than progressively. While the evidence presented is in the form of observation, and no direct measurements of brain stem reticular activitywere made, the close association between changes in ventilation and EEG support the argument for an interactive control of arousal and respiration by reticular structures within the brrain stem.

\subsection{Clinical implications}

The rapidity and magnitude of the decrease in ventilation and the rise in the end-tidal levels of oxygen have implications for the hypoventilation sometimes observed in NREM sleep, most seriously evidenced as apnea. Dempsey and Skatrud (1983) hypothesise that the apneic threshold for $\mathrm{CO}_{2}$ is dependent on the overall level of afferent input to the ventilatory control system. In the same article, they go on to say that " in the absence of the stabilizing influences of wakefulness, even the healthy person is vulnerable to instabilities and ventilatory control as mainteneance of a rhythmic breathing pattern becomes overwhelmingly dependent on $\mathrm{CO}_{2}$ " (Dempsey \& Skatrud, 1983). 
Such a relationship would predict that the magnitude and rapidity of the decrease in ventilation with sleep may in some circumstances be sufficient to produce apnea. A large amount of tonic afferent activity provided to the ventilatory control system by a wakefulness drive could produce a state of mild hypocapnia. Given that the $\mathrm{CO}_{2}$ inspiration threshold is known to increase with the onset of NREM sleep, in the early stages of NREM the $\mathrm{P}_{\mathrm{A}} \mathrm{CO}_{2}$ level could be well below the new sleep threshold and lead to some period of apnea ( as ventilatory control is soley determined by $\mathrm{CO}_{2}$ levels during NREM sleep). Two possible courses of events could follow such an apnea. The condition could be followed by a small number of large (augmented) breaths, which would again drive $\mathrm{CO}_{2}$ levels below the inspiration threshold, and another period of apnea would then occur. This is of course the pattern of respiration known as periodic breathing. A second possibility is that the initial apnea results in an arousal response rather than an augmented inspiration response, or that the augmented inspiration litself leads to a re-arousal. This would be a cause of sleep onset insomnia, where the sufferer has difficulty attaining a state of stable NREM sleep.

It could be hypothesised that the larger the tonic afferent input to ventilation provided by wakefulness (wakefulness drive) the more susceptible a person would be to either or both of the above problems. That is, the larger the degree of hypocapnia, the longer the period reqired for an inspiration threshold to be reached. While this hypothesis needs to be tested experimentally (by artifically increasing wake arousal levels in normal subjects) and in clinical populations, the data of the thesis provide evidence for a usefull model to explain the cause of some respiratory-related sleep pathology. 


\subsection{Summary}

The data from the three experiments of the present thesis have provided conclusive evidence that the point of theta onset is consistently related to decreases in ventilation and changes in end tidal gas levels. This relationship was found in almost every sleep onset in both males and females, and the female result was not affected by menstrual phase. Further the relationship was independent of the method used to measure ventilation and was thus not influenced by equipment artifact. The interpretation of these results has been that the ventilation decrease is due to the cessation of a wakefulness drive.

A qualification of this interpretation is that the changes in ventilation may be due to the increase in upper airway airway resistance known to be associated with NREM sleep in animals and man. However, as already commented, Rist et al. (1986) have shown that during sleep, reduced mechanical efficiency accounts for only a portion of the total reduction in ventilation, the other factors being reduced central respiratory neural output and reduced MR. Further, there is evidence in animals which suggests that ventilation decreases occur with sleep, despite the maintenance of a patent airway (Phillipson et al., 1976; Phillipson et al., 1978; Sullivan et al., 1978). It remains to be determined whether or not increased airway resistance occurs during the period of sleep onset in humans or to what extent it can account for the ventilation decrease during this time. 
REFERENCES 
Askanazi J., P.A. Silverberg, R.J. Foster, A.I. Hyman, J. Milic-Emili, J.M. Kinney. Effects of respiratory apparatus on breathing pattern. J. Appl. Physiol. 48(4): 577-580, 1980.

Asmussen, E. Regulation of respiration: "The black box". Acta Physiol. Scand. 99: 85-90, 1977.

Bäckström, T., H. Carstensen \& R. Södergård. Concentration of estradiol, testosterone and progesterone in cerebrospinal fluid compared to plasma unbound and total concentrations. J. Steroid. Biochem. 7: 469 -472, 1976.

Bainton, C.R. \& R. A. Mitchell. Posthyperventilation apnea in awake man. J. Appl. Physiol. 21(2): 411-415, 1966.

Bellville, J.W., W.S. Howland, J.C. Steed \& R.W. Houde. The effect of sleep on the respiratory response to carbon dioxide. Anesthesiology 20(5): 628-634, 1959.

Berger, A.J., R.A. Mitchell \& J.W. Severinghaus. Regulation of respiration (First of three parts). New Engl. J. Med. 297: 92-97, 1977a.

Berger, A.J., R.A. Mitchell \& J.W. Severinghaus. Regulation of respiration (Second of three parts). New Engl. J. Med. 297: 138 - 143, 1977 b.

Berger, A.J., R.A. Mitchell \& J.W. Severinghaus. Regulation of respiration (Third of three parts). New Engl. J. Med. 297: 194-201, 1977 c.

Berthon-Jones M., C.E. Sullivan. Ventilatory and arousal responses to hypoxia in sleeping humans. Am. Rev. Respir. Dis. 125: 632 - 639, 1982. 
Berthon-Jones, M., C.E. Sullivan. Ventilation and arousal responses to hypercapnia in normal sleeping humans. J. Appl. Physiol. 57(1): 59 - 67, 1984.

Birchfield, R.I., H.O. Sieker \& A. Heyman. Alterations in respiratory function during natural sleep. J. Lab. \& Clin. Med. 54(2): 216-222, 1959.

Block A.J., P.G. Boysen, J.W. Wynne, L.A. Hunt. Sleep apnea, hypopnea and oxygen desaturation in normal subjects ( A strong male predominance). N. Eng. J. Med. 300(10): 513 - 517, 1979.

Block A.J., D.W. Hellard \& P.C. Slayton. Alcohol ingestion has minimal effects on breathing during sleep of postmenopausal women. Am. Rev. Respir. Dis. 131: A101, 1985.

Block A.J., D.W. Hellard \& P.C. Slayton. Effect of alcohol ingestion on breathing and oxygenation during sleep: analysis of the influence of age and sex. Am. J. Med. 80: 595-600, 1986.

Block A.J., J.W. Wynne \& P.G. Boysen. Sleep disordered breathing and nocturnal oxygen saturation in postmenopausal women. Am. J. Med. 69: 75 - 79, 1980.

Botella-Llusiá. Endochrinology of Woman. Translated by E.A. Moscovic.

W.B. Saunders Co., Philadelphia, 1973.

Brebbia, D.R. \& K.Z. Altshuler. Oxygen consumption rate and electroencephalographic stage of sleep. Science 150: 1621-1623, 1965.

Bulow, K. Respiration and wakefulness in man. Acta Physiol. Scand. 59: [Suppl. 209] $1-110,1963$. 
Bulow, K.\& D.H. Ingvar. Respiration and state of wakefulness in normals studied by spirography, capnography and EEG. Acta Physiol. Scand. 51: 230-238, 1961.

Catterall, J.R., P.M.A. Calverley, C.M. Shapiro, D.C. Flenley \& N.J. Douglas. Breathing and oxygenation during sleep are similar in normal men and normal women. Am. Rev. Respir. Dis. 132: 86 - 88, 1985.

Cherniack, N.S. Respiratory dysrythmias during sleep. New Engl. J. Med. 305(6): 325-330, 1981.

Chiodi H., H.D. Snow. Action of progesterone on respiratory exchanges of goats. Fed. Proc. 30: 327, A256, 1971.

Coccagna, G. \& E. Lugaresi. Arterial blood gasses and pulmonary and systemic arterial pressure during sleep in chronic obstructive pulmonary disease. Sleep 1(2): 117-124, 1978.

Cohen, M.I. Central determinants of respiratory rhythm. Ann. Rev. Physiol. 43: 91-104, 1981.

Comroe, J.H. Physiology of Respiration 2nd Ed. Year Book Medical Publishers Inc., Chicago, 1974.

Coote, J.H. Respiratory and circulatory control during sleep. J. Exp. Biol. 100: 223-244, 1982.

Cugell, D.W., N.R. Frank, E.A. Gaensler \& T.L. Badger. Pulmomary function in pregnancy. Am. Rev. Respir. Dis. 67: 568-597, 1953. 
Cullen, J.H., V.C. Brum \& W.V. Reidt. The respiratory effects of progesterone in severe pulmonary emphysema. Am. J. Med. Oct: 551 - 557, 1959.

Dempsey, J .A., J. B. Skatrud. A sleep-induced apneic thresholld and its consequences. Am. Rev. Resp. Dis. 133; 1163-1170

Douglas, N.J. Control of breathing during sleep. Clin. Sci. 67: 465-471, 1984.

Douglas, N.J., D.P. White, C.K. Pickett, J.V. Weil \& C.W. Zwillich. Respiration during sleep in normal man. Thorax 37: 840-844, 1982a.

Douglas N.J., D.P. White, J.V. Weil, C.K. Pickett, R.J. Martin, D.W. Hudgel \& C.W. Zwillich. Hypoxic ventilatory response decreases during sleep in normal men. Am. Rev. Resp. Dis 125: 286-289, 1982 b.

Douglas, N.J., D.P. White, J.V. Weil, C.K. Pickett \& C.W. Zwillich. Hypercapnic ventilatory response in sleeping adults. Am. Rev. Respir. Dis. 126: 758-762, 1982c.

Duron, B. Respiratory function during phsyiological sleep. Bull. Physio-path. Resp. 8: 1031-1057, 1972.

Duron, B., C. Andrac \& P. Laval. Ventilation pulmonaire globale $\mathrm{CO} 2$ alvéolaire et consommation d'oxygene au cours du sommeil chez l'Homme normal. Société de Biologie de Marseille Décembre: 139-145, 1967.

Duron, B., D. Marlot. Intercostal and diaphragmatic electrical activity during wakefulness and sleep in normal unrestrained adult cats. Sleep 3(3/4): 269-280, 1980. 
Eldridge, F.L. Posthyperventilation breathing: different effects of active and passive hyperventilation. J. Appl. Physiol. 34(4): 422-430, 1973.

England, S.J. \& L.E. Farhi. Fluctuations in alveolar $\mathrm{CO} 2$ and in base excess during the menstrual cycle. Resp. Physiol. 26: 157-161, 1976.

Fink, B.R. Influence of cerebral activity in wakefulness on regulation of breathing. J. Appl. Physiol. 16(1): 15-20, 1961.

Gilbert R., J.H. Auchincloss Jr., J. Brodsky, W.Boden. Changes in tidal volume, frequency, and ventilation induced by their measurement. J. Appl. Physiol. 33(2): $252-254,1972$.

Gleeson K., C.W. Zwillich, K. Braier \& D.P. White. Breathing route during sleep. Am. Rev. Respir. Dis. 134: 115-120, 1986.

Goldie, L. \& J.M. Green. Changes in mode of respiration an indication of level of awareness. Nature 189: 581-582, 1961.

Goldman, M.D. Interpretation of thoracoabdominal movements during breathing. Clin. Sci. 62: 7-11, 1982.

Goodland, R. L., J.G. Reynolds, A.B. McCoord \& W.T. Pommerenke. Respiratory and electrolyte effects induced by estrogen and progesterone. Fertil. Steril. 4: 300 - 317, 1953.

Gothe, B., M.D. Altose, M.D. Goldman \& N.S. Cherniack. Effect of quiet sleep on resting and CO2-stimulated breathing in humans. J. Appl. Physiol. 50(4): 724-730, 1981. 
Gothe, B., N.S. Cherniack \& L.Williams. Effect of hypoxia on ventilatory and arousal responses to $\mathrm{CO} 2$ during NREM sleep with and without Flurazepam in young adults. Sleep. 9(1): 24-37, 1986.

Gothe B., M.D. Goldman, N.S. Cherniack \& P. Mantey. Effect of progressive hypoxia on breathing during sleep. Am. Rev. Respir. Dis. 126: 97-102, 1982.

Greig, M., M.G. Coyle, W.Cooper \& J. Walker. Plasma progesterone in mother and fetus in the second half of human pregnancy. J. Obst. Gynaecol. Brit. Common. 69: 772 - 776, 1962.

Guazzi, G., E.D. Freis. Sino-aortic reflexes and arterial pH, pO2 and pCO2 in wakefulness and sleep. Am. J. Physiol. 217(6): 1623-1627, 1969.

Guilleminault,C., J. van der Hoed \& M.M. Mitler. Clinical overview of the sleep apnea syndromes. In Sleep Apnea Syndromes, Edited by Guilleminault, C. \& W.C. Dement, Allan R. Liss Inc., New York, 1978, p. 1-12.

Harman, E., J.W. Wynne, A.J. Block \& L. Malloy-Fisher. Sleep-disordered breathing and oxygen saturation in obese patients. Chest 79(3): $256-260,1981$.

Haskell, E.H., J.W. Palca, J.M. Walker, R.J. Berger \& H.C. Heller. Metabolism and thermoregulation during stages of sleep in humans exposed to heat and cold. J. Appl. Physiol. 51(4): 948-954, 1981.

Hedemark, L.L. \& R.S. Kronenberg. Ventilatory and heart rate responses to hypoxia and hypercapnia during sleep in adults. J. Appl. Physiol. 53(2): 307-312, 1982. 
Hellegers, A., J. Metcalfe, W.E. Huckabee, H. Prytowsky, G. Meschia \&

D.H. Barron. Alveolar PCO2 and PO2 in pregnant and nonpregnant women at high altitude. Am. J. Obstet. and Gynecol. 82(2): 241 - 245, 1961.

Hensley, M.J., N.A. Saunders \& K.P. Strohl. Medroxyprogesterone treatment of obstructive sleep apnea. Sleep 3(3/4): 441-446, 1980.

Hirsch. J.A. \& B.Bishop. Human breathing patterns on mouthpiece or face mask during air, CO2, or low O2.J. Appl. Physiol. 53(5): 1281-1290, 1982.

Hirshman, C.A., R.E. McCullough \& J.V. Weil. Normal values for hypoxic and hypercapnic ventilatory drives in man. J. Appl. Physiol. 38(6): 1095 - 1098, 1975.

Honda, Y., F. Hayashi, A. Yoshida, Y. Ohyabu, Y. Nishibayashi \& H. Kimura. Overall "gain" of the respiratory control system in normoxic humans awake and asleep. J. Appl. Physiol. 55(5): 1530-1535, 1983.

Hudgel, D.W. \& P. Devadatta. Decrease in functional residual capacity during sleep in normal humans. J. Appl. Physiol. 57(5): 1319-1322, 1984.

Hudgel, D.W., R.J. Martin, B. Johnson \& P. Hill. Mechanics of the respiratory system and breathing pattern during sleep in normal humans. J. Appl. Physiol. 56(1): 133-137, 1984.

Hugelin, A. \& M.I. Cohen. The reticular activating system and respiratory regulation in the cat. Annals New York Acad. Sci. 109(2): 586-603, 1963. 
Irsigler, G.B. Carbon dioxide response lines in young adults: the limits of the normal response. Am. Rev. Respir. Dis. 114: 529 - 536, 1976.

Ito, H. \& D.M. Aviado. Prevention of pulmonary emphysema in rats by progesterone. J. Pharmacol. \& Exper. Therapeutics. 161(2): 197 - 205, 1968.

Johnson, M.W., A.M. Anch, J.E. Remmers. Induction of the obstructive sleep apnea syndrome in a woman by exogenous androgen administration. Am. Rev. Resp. Dis. 129: 1023-1025, 1984.

Khatri, I.M. \& E.D. Freis. Hemodynamic changes during sleep. J. Appl. Physiol. 22(5): 867-873, 1967.

Kopelman P.G., M.C.P. Apps, T. Cope \& D.W. Empey. The influence of menstrual status, body weight and hypothalamic function on nocturnal respiration in women. J. Royal Coll. Physicians Lond. 19(4): 243-247, 1985.

Kreider, M.B. \& P.F. Iampietro. Oxygen consumption and body temperature during sleep in cold environments. J. Appl. Physiol. 14(5): 765-767, 1959.

Krieger, J., J. Turlot, P. Mangin \& D. Kurtz. Breathing during sleep in normal young and elderly subjects: hypopneas, apneas and correlated findings. Sleep 6(2): 108-120, 1983.

Krol, R.C., S.L. Knuth \& D. Bartlett. Selective reduction of genioglossal muscle activity by alcohol in normal human subjects. Am. Rev. Respir. Dis. 129: 247-250, 1984. 
Kryger, M., R. Glas, D. Jackson, R.E. McCullough, C. Scoggin, R.F. Grover \& J.V. Weil. Impaired oxygenation during sleep in excessive polycythemia of high altitude: improvement with respiratory stimulation. Sleep 1: 3 - 17, 1978.

Kryger, M., R.E.McCullough, D. Collins, C.H. Scoggin, J.V. Weil \& R.F. Grover. Treatment of excessive polycythemia of high altitude with respiratory stimulant drugs. Am. Rev. Respir. Dis. 117: 455 - 464, 1978.

Kumar, A., R. van Diest, W.F. Hofman, P. Visser, P.A.M. Poelstra \& H.J. Bakker. Sleep state dependent parameters of respiration in man. In Sleep 1978: Fourth Eurpoean Conference on Sleep Research. Edited by Popoviviu, L., B. Asgian \& G Badia. S. Karger, Basel, 1980.

Leiter, J.C., E.A. Doble, S.L. Knuth \& D. Bartlett Jr. Respiratory activity of genioglossus: interaction between alcohol and the menstrual cycle. Am Rev Respir Dis 135: 383-386, 1987.

Llewellyn-Jones, D. Fundamentals of Obstetrics and Gynaecology. Vol. II. Faber \& Faber, London, 1970.

Lopes, J.M., E. Tabachnik, N.L. Muller, H. Levison, A.C. Bryan. Total airway resistance and respiratory muscle activity during sleep. J. Appl. Physiol 54(3): 773-777, 1983.

Lugaresi, E., G. Coccagna, F. Cirignotta, P. Farneti, R. Gallassi, G. Di Donato, P. Verucchi. Breathing during sleep in man in normal and pathological conditions. Advances in Exp. Med. \& Biol. 99: 35-45, 1978. 
Lurie, A.O. \& J.B. Weiss. Progesterone in cerebrospinal fluid during human pregnancy. Nature 215(9): 1178, 1967.

Lyons, H. \& R. Antonio. The sensitivity of the respiratory center in pregnancy and after administration of progesterone. Trans. Assoc. Am. Phys. 72: 173 - 180, 1959.

Lyons, H.A. \& C.T. Huang. Therapeutic use of progesterone in alveolar hypoventilation associated with obesity. Am. J. Med. 44: 881 - 888, 1968.

Machida, H. Influence of progesterone on arterial blood and CSF acid-base balance in women. J. Appl. Physiol. 51(6): 1433-1436, 1981.

Magnussen, G. Studies on the respiration during sleep. H.K. Lewis \& Co. Ltd. London, 1944.

Mayevsky, A. \& D. Samuel. The use of 1802 in studying oxygen metabolism in various behavioral situation. J.Neurosci. Res. 1: 495-499, 1975.

Megirian, D., R. Cespuglio \& M. Jouvet. Rhythmical activity of the rat's tongue in sleep and wakefulness. Electroenceph. Clin. Neurophys. 44: 8-13, 1978.

Megirian, D., J. H. Sherrey. Respiratory functions of the laryngeal muscles during sleep. Sleep 3(3/4): 289-298, 1980.

Mei, S.S., D. Gort \& F.F. Kao. The invetsiagtion of respiratory effects of progesterone in cross-circulated dogs. Fed. Proc. 36: 489, 1977.

Mitchell, R.A., C.R. Bainton \& G. Edelist. Posthyperventilation apnea in awake dogs during metabolic acidosis and hypoxia. J. Appl. Physiol. 21(4): 1363-1367, 1966. 
Moore, L.G., R.E. McCullough \& J.V. Weil. Increased HVR in preganacy: relationship to hormonal and metabolic changes. J. Appl. Physiol. 62(1): 158 - 163, 1987.

Morrison, D.A. \& A.L. Goldman. Oral progesterone therapy in COPD. Am. Rev. Respir. Dis. 119: 154, 1979.

Mortola, J.P.\& A.M. Anch. Chest wall configuration in supine man: wakefulness and sleep. Resp. Physiol. 35: 201-213, 1978.

Naifeh K.H. \& J. Kamiya. The nature of respiratory changes associated with sleep onset. Sleep. 4(1): 49-59, 1981.

Naifeh, K.H., J. Kamiya \& D. Monroe-Sweet. Biofeedback of alveolar carbon dioxide tension and levels of arousal. Biofeedback and Self Regulation 7(3): 283-299, 1982.

Naifeh K.H., J.W. Severinghaus \& J. Kamiya. Effect of aging on estimates of hypercapnic ventilatory response during sleep. Sleep Research 16: 52, 1987.

Newsom-Davis, J., L. Loh, J. Nodal \& M. Charnock. Effects of sleep on the pattern of $\mathrm{CO} 2$ stimulated breathing in males and females. In The Regulation of Respiration during Sleep and Anesthesia. Edited by Fitzgerald, R.S., H. Gautier \& S, Lahiri. Plenum. New York, 1978.

Ogilvie R.D. \& R. T. Wilkinson. The detection of sleep onset: behavioral and physiological convergence. Psychophysiology 21(5): 510-520, 1984. 
Orem, J.M. Central neural interactions between sleep and breathing. In $\underline{\text { Sleep and }}$ Breathing Edited by Saunders, N.A, \& C.E. Sullivan, Marcel Dekker, Inc. New York, 1984, p. $91-136$.

Orem, J. \& W.C. Dement. Neurophysiological substrates of the changes in respiration during sleep. In Advances in Sleep Research Vol 2. Edited by Weitzman, E.D. Spectrum Publications, New York, 1975, p. 1-42.

Orem, J.\& R. Lydic. Upper airway function during sleep and wakefulness: experimental studies on normal and anesthetized cats. Sleep 1: 49-68, 1978.

Orem, J., J. Montplaisir \& W.C. Dement. Changes in the activity of respiratory neurons during sleep. Brain Research. 82: 309-315, 1974.

Orem, J., A. Netick \& W.C. Dement. Breathing during sleep and wakefulness in the cat. Respir. Physiol. 30: 265-289, 1977a.

Orem, J., A. Netick \&W. C. Dement. Increased upper airway resistance to breathing during sleep in the cat. Electroenceph. Clin. Neuro. 43: 14-22, $1977 \mathrm{~b}$.

Orr, W.C. Sleep and breathing: an overview. Ear Nose \& Throat J. 63: 10-21, 1984.

Orr, W.C., N.K. Imes \& R.J. Martin. Progesterone therapy in obese patients with sleep apnea. Ann. Intern. Med. 139: 109 - 111, 1979.

Pappenheimer, J.R. Sleep and respiration of rats during hypoxia. J. Physiol. 266: 191-207, 1977. 
Parmeggiani, R.L. Integrative aspects of hypothalamic influences on respiratory brain stem mechanisms during wakefulness and sleep. In Central Nervous Control Mechanisms in Breathing Edited by von Euler, C. \& H. Lagercrantz. Pergamon Press, Oxford, 1979, p. 53 - 70.

Parmeggiani, R.L. \& L. Sabattini. Electromyographic aspects of postural, respiratory and thermoregulatory mechanisms in sleeping cats. Electroenceph.\& Clin. Neuro. 33: $1-13,1972$.

Patrick, J.M. \& A. Howard. The influence of age, sex, body size and lung size on the control and pattern of breathing during $\mathrm{CO} 2$ inhalation in caucasians. Resp. Physiol. 16: $337-350,1972$.

Pernoll, M.L., J. Metcalfe, P.A. Kovach, R. Wachtel \& M.J. Dunham. Ventilation during rest and exercise in pregnancy and postpartum. Respir. Physiol. 25: 295 - 310, 1975.

Peset, F.G. Changes in oxygen saturation and heart frequency during sleep in young normal subjects. Thorax 39: 673-675, 1984.

Phillipson, E.A. Regulation of breathing during sleep. Am. Rev. Resp. Dis. 6(2): 217-224, 1977.

Phillipson E.A. Control of breathing during sleep. Am. Rev. Resp. Dis. 118: 909-939, 1978. 
Phillipson, E.A., G. Bowes. Control of breathing during sleep. In Handbook of Physiology. 1986

Phillipson, E.A., G. Bowes, E.R. Townsend, J. Duffin \& J.D. Cooper.

Role of metabolic $\mathrm{CO} 2$ production in ventilatory response to steady-state exercise.

J. Clin. Invest. 68: 768-774, 1981.

Phillipson, E.A., J. Duffin \& J.D. Cooper. Critical dependence of respiratory rhythmicity on metabolic CO2 load. J. Appl. Physiol. 50(1): 45-54, 1981.

Phillipson, E.A., E. Murphy \& L.F. Kozar. Regulation of respiration in sleeping dogs. J. Appl. Physiol. 40(5): 688-693, 1976.

Phillipson, E.A., C.E. Sullivan, J.C. Read, E. Murphy \& L.F. Kozar. Ventilatory and waking responses to hypoxia in sleeping dogs. J. Appl. Physiol. 44(4): 512 - 520, 1978.

Plass, E.D., F.W. Oberst. Respiration and pulmonary ventilation in normal nonpregnant, pregnant an puerperal women. Am. J. Obs. Gyn. 35: 441-452, 1938.

Plum F. Neurological integration of behavioural and metabolic control of breathing. In Breathing: Hering-Breuer Centenary Symposium. Edited by Porter, R. Churchill, London, 1970, p. 159-181.

Plum, F. Cerebral control of breathing. In Ventilatory and Phonatory Control Systems Edited by Wyke, B. Oxford University Press, London, 1974. p. 208-223. 
Rechtshaffen, A., A.Kales. A Manual of Standardised Terminology. Techniques and Scoring System for Sleep Stages of Human Subjects. Washington D.C. Natl. Inst. Health, 1968 (publ. 204).

Reed, D.J. \& R.H. Kellogg. Changes in respiratory response to $\mathrm{CO} 2$ during natural sleep at sea level and at altitude. J. Appl. Physiol. 13(3): 325-330, 1958.

Reed, D.J.\& R.H. Kellogg. Effect of sleep on hypoxic stimulation of breathing at sea level and altitude. J.Appl. Physiol 15(6): 1130-1134, 1960a.

Reed, D.J. \& R.H. Kellogg. Effect of sleep on $\mathrm{CO} 2$ stimulation of breathing in acute and chronic hypoxia. J. Appl. Physiol. 15(6): 1135-1138, $1960 \mathrm{~b}$.

Remmers, J.E. Effects of sleep on control of breathing. In Respiratory Physiology III Vol. 23 Edited by J.G. Widdicombe University Park Press, Baltimore, 1981.

Remmers, J.E., D. Bartlett \& M.D. Putnam. Changes in the respiratory cycle associated with sleep. Respir. Physiol. 28: 227-238, 1976.

Rist K. E., J. A. Daubenspeck, J.F. McGovern. Effects of non-REM sleep upon respiratory drive and the respiratory pump in humans. Resp. Physiol. 63: 241-256, 1986.

Robin, E.D., R.D. Whaley, C.H. Crump \& D.H. Travis. Alveolar gas tensions, pulmonary ventilation and blood $\mathrm{pH}$ during physiologic sleep in normal subjects. J.Clin. Invest. 37: 981-989, 1958. 
Sackner, J.D., A.J. Nixon, B.Davis, N. Atkins \& M.A. Sackner. Effects of breathing through external dead space on ventilation at rest and during exercise. Am. Rev. Resp. Dis. 122: 933-940, 1980.

Sandblom, R.E., A.V. Matsumoto, R.B. Schoene, K.A. Lee, E.C. Giblin, W.J. Bremner \& D.J. Pierson. Obstructive sleep apnea syndrome induced by testosterone administration. N. Engl. J. Med. 308(9): 508-510, 1983.

Santiago, T.V., A.K. Shina \& N.M. Edelman. The respiratory responses to continuous inspiratory flow resistive loading of sleeping cats. Proc. Fed. Am. Soc. Exp. Biol. 38(2): 1299, A5673, 1979.

Saunders, N.A., S. Heilpern \& A.S. Rebuck. Relation between personality and ventilatory respose to carbon dixide in normal subjects: A role in asthma? $\underline{\mathrm{Br} . \text { Med. J. }}$ 1: $719-721,1972$.

Schmidt, R.F. Fundamentals of Neurophysiology Spinger-Verlag, New York, 1978.

Schoene, R.B., D.J. Pierson, S. Lakshminarayan, D.L. Shrader \& J. Butler. Effect of medroxyprogesterone acetate on respiratory drives and occlusion pressure. Bull. Europ. Physiopath. Resp. 16: 645 - 653, 1980.

Schoene, R.B., H.T. Robertson, D.J. Pierson \& A.P.Peterson. Respiratory drives and exercise in menstrual cycles of athletic and nonathletic women. J. Appl. Physiol. 50: 1300-1305, 1981. 
Shapiro, C.M., C.C. Goll, G.R. Cohen \& I. Oswald. Heat production during sleep. J. Appl. Physiol. 56(3): 671-677, 1984.

Sharp, J.T., N.B. Goldberg, W.S. Druz \& J. Danon. Relative contributions of rib cage and abdomen to breathing in normal subjects. J. Appl. Physiol. 39(4): 608-618, 1975.

Shea, S.A., J. Walter, C. Pelley, K. Murphy \& A. Guz. The effect of visual and auditory stimuli upon resting ventilation in man. Respir. Physiol. 68: 345-357, 1987.

Sherrey, J.H. \& D. Megirian. State dependence of upper airway respiratory motoneurons: functions of cricothyroid and nasolabial muscles of the unanesthetized rat. Electroenceph. Clin. Neurophys. 43: 218-228, 1977.

Sherrey, J. \& D. Megirian. Respiratory EMG activity of the posterior cricoarytenoid, cricothyroid and diaphragm muscles during sleep. Respir. Physiol. 39: 355-365, 1980. Shore, E.T., R.P. Millman, D.A. Silage, D.C. Chung \& A.I. Pack. Ventilatory and arousal patterns during sleep in normal young and elderly subjects. $\underline{\text { J. Appl. Physiol. }}$ 59(5): 1607-1615, 1985.

Skatrud, J.B., A.D. Berssenbrugge. Effect of sleep state and chemical stimuli on breathing. Prog. Clin. Biol. Res. (U.S) 136: 87-95, 1983.

Skatrud J.B.\& J.A. Dempsey. Interaction of sleep state and chemical stimuli in sustaining rhythmic ventilation. J. Appl. Physiol. 55(3): 813-822, 1983. 
Skatrud, J.B., J.A. Dempsey \& D.G. Kaiser. Ventilatory response to medroxyprogesterone acetate in normal subjects: time course and mechanism. J. Appl. Physiol. 44(6): 939 - 944, 1978.

Snyder, F., J.A. Hobson, D.F. Morrison \& F. Goldfrank. Changes in respiration, heart rate, and systolic blood pressure in human sleep. J. Appl. Physiol. 19(3): 417-422, 1964.

St. John, W.M. Influence of reticular mechanisms upon hypoglossal trigeminal and phrenic activities. Respir. Physiol. 66: 27-40, 1986.

St. John, W.M., D. Bartlett, K.V. Knuth, S.L. Knuth \& J. A. Daubenspeck. Differential depression of hypoglossal nerve activity by alcohol: Protection by pretreatment with medroxyprogesterone acetate. Am. Rev. Resp. Dis. 133: 46-48, 1986.

Stahl, M.L., W.C. Orr \& J.L. Males. Progesterone levels and sleep-related breathing during menstrual cycles in normal women. Sleep 8(3): 227 - 230, 1985.

Stoney, C.M., A.W. Langer \& P.D. Gelling. The effects of menstrual cycle phase on cardiovascular and pulmonary responses to behavioral and exercise stress. Psychophysiology 23(4): 393 - 402, 1986.

Strohl, K.P., M.J. Hensley, N.A. Saunders, S.M. Scharf, R.Brown \& R.H. Ingram. Progesterone administration and progressive sleep apneas. J. Am. Med. Assoc. 245(12): $1230-1232,1981$. 
Sullivan C.E. Breathing in sleep. In Physiology in sleep. Edited by Orem J. and C.D. Barnes. New York: Academic Press, 1980. p 213-272.

Sullivan, C.E., L.F. Kozar, E. Murphy \& E.A. Phillipson. Primary role of respiratory afferents in sustaining breathing rhythm. J. Appl. Phys. 45(1): 11-17, 1978.

Sutton, F.D., C.W. Zwillich, C.E. Creagh, S.J. Pierson \& J.V. Weil. Progesterone for outpatient treatment of Pickwickian syndrome. Ann. Intern. Med. 83; 476 - 479, 1975.

Taasan, V.C., A.J. Block, P.G. Boysen, J.W. Wynne, C. White \& S. Lindsey. Alcohol increases sleep apnea and oxygen desaturation in asymptomatic men. Am. J. Med. 71: 240- 245, 1981

Tabachnik E., N.L. Muller, A.C. Bryan \& H. Levison. Changes in ventilation and chest wall mechanics during sleep in normal adolescents. J. Appl. Physiol. 51(3): 557-564, 1981.

Takano, N. Reflex hypoxic drive to respiration during the menstrual cycle. Respir. Physiol. 56: 229 - 235, 1984.

Takano, N., A. Sakai \& Y. lida. Analysis of alveolar PCO2 control during the menstrual cycle. Pflügers Arch. 390: 56 - 62, 1981.

Tawadrous, F.D. \& F.L.Eldridge. Posthyperventilation breathing patterns after active hyperventilation in man. J. Appl. Physiol. 37(3): 353-356, 1974.

Timmons, B., J. Salamy, J. Kamiya \& D. Girton. Abdominal-thoracic respiratory movements and levels of arousal. Psychonomic Sci. 27(3): 173-175, 1972. 
Tok, G. \& H.H. Loeschcke. The effect of progesterone on the medullary respiratory chemosensitivity in the cat. Pflügers Archiv. 382: R18(Suppl:), 1975.

Townsend, R.E., P.N. Prinz \& W.D. Obrist. Human cerebral blood flow during sleep and waking. J. Appl. Physiol. 35(5): 620-625, 1973.

Tusiewicz, K., Moldofsky, A.C. Bryan \& M.H. Bryan. Mechanics of the rib cage and diaphragm during sleep. J. Appl. Physiol. 43(4): 600-602, 1977.

Tyler, J.M. The effect of progesterone on the respiration of patients with emphysema and hypercapnia.J. Clin. Invest. 39: 34 - 41, 1960.

Vander, A.J., J.H. Sherman \& D.S. Luciano. Human Physiology - The Mechanisms of Body Function. McGraw-Hill Book Co., New York, 1975.

Vincent S.B., The function of vibrissae in the behavior of the white rat. Behav. Monog. 1: 1912.

Webb, P. \& M. Hiestand. Sleep metabolism and age. J. Appl. Physiol. 38(2): 257-262, 1975.

Weissman, C., J. Askanazi, J. Milic-Emili \& J.M. Kinney. Effect of respiratory apparatus on respiration. J. Appl. Physiol. 57(2): 475-480, 1984.

White, D.P. Central sleep apnea. Med. Clinics Nth. Am. 69(6): 1205-1219, 1985. 
White, D.P. Occlusion pressure and ventilation during sleep in normal humans. J. Appl. Physiol. 61(4): 1279-1287, 1986.

White D.P., N.J. Douglas, C.K. Pickett, J.V. Weil. \& C.W. Zwillich. Hypoxic ventilatory response during sleep in normal premenopausal women. Am. Rev. Respir. Dis. 126: 530 - 533, 1982.

White, D.P., N.J. Douglas, C.K. Pickett, J.V. Weil \& C.W. Zwillich. Sexual influence on the control of breathing. J. Appl. Physiol. 54(4): 874 - 879, 1983.

White, D.P., B.K. Schneider, R.J. Santeen, M.McDermott, C.K. Pickett, C.L. Zwillich \& J.V. Weil . Influence of testosterone on ventilation and chemosenstitivty in male subjects. J. Appl. Physiol. 59(5): 1452-1457, 1985a.

White D.P., J.V. Weil \& C.W. Zwillich. Metabolic rate and breathing during sleep. J. Appl. Physiol. 59(2): 384-391, 1985 b.

Yamashiro Y., Y. Fukushima, N. Okudaira, Y. Suzuku \& I. Nishi. Gas exchange ratio during sleep. Sleep Research 16: 60, 1987.

Zwillich, C.W., M.R. Natalino, F.D. Sutton \& J.V. Weil. Effects of progesterone on chemosensitivity in normal men. J. Lab. Clin. Med. 92(2): 262-269, 1978. 


\section{APPENDIX 1}

\section{List of Papers from the Thesis}

Colrain, I.M., J.A. Trinder, G.J., Fraser \& G.V. Wilson Ventilation During Sleep Onset. J. Appl. Physiol. 63(5): 2067 - 2074, 1987. 
Appendix 2

Standard deviation values for Table 6.1

Wake Transitions in Quartiles Sleep

\begin{tabular}{|c|c|c|c|c|c|c|c|}
\hline Y.B. & $\mathrm{VE}$ & 0.596 & 0.631 & 0.417 & 0.529 & 0.499 & 0.432 \\
\hline \multirow[t]{3}{*}{$\mathrm{N}=18$} & $\mathrm{VO}_{2}$ & 0.039 & & & & & 0.033 \\
\hline & $\mathrm{FetO}_{2}$ & 0.65 & 0.71 & 0.67 & 0.64 & 0.66 & 0.64 \\
\hline & $\mathrm{VE} / \mathrm{VO}_{2}$ & 5.75 & & & & & 4.85 \\
\hline J.B. & VE & 0.592 & 0.559 & 0.561 & 0.494 & 0.472 & 0.582 \\
\hline \multirow[t]{3}{*}{$\mathrm{N}=20$} & $\mathrm{VO}_{2}$ & 0.029 & & & & & 0.032 \\
\hline & $\mathrm{FetO}_{2}$ & 0.43 & 0.42 & 0.50 & 0.47 & 0.39 & 0.38 \\
\hline & $\mathrm{VE} / \mathrm{VO}_{2}$ & 2.66 & & & & & 2.46 \\
\hline J.S. & $\mathrm{VE}$ & 0.746 & 0.810 & 0.797 & 0.629 & 0.704 & 0.528 \\
\hline \multirow[t]{3}{*}{$\mathrm{N}=25$} & $\mathrm{VO}_{2}$ & 0.030 & & & & & 0.020 \\
\hline & $\mathrm{FetO}_{2}$ & 0.42 & 0.38 & 0.47 & 0.41 & 0.46 & 0.44 \\
\hline & $\mathrm{VE} / \mathrm{VO}_{2}$ & 2.56 & & & & & 1.24 \\
\hline G.O. & VE & 0.727 & 0.795 & 0.814 & 0.698 & 0.348 & 0.605 \\
\hline \multirow[t]{3}{*}{$\mathrm{N}=22$} & $\mathrm{VO}_{2}$ & 0.035 & & & & & 0.033 \\
\hline & $\mathrm{FetO}_{2}$ & 0.30 & 0.33 & 0.29 & 0.31 & 0.32 & 0.33 \\
\hline & $\mathrm{VE} / \mathrm{VO}_{2}$ & 2.83 & & & & & 2.78 \\
\hline
\end{tabular}

$\begin{array}{llllllll}\text { K.M. } & \text { VE } & 0.718 & 0.746 & 0.693 & 0.599 & 0.631 & 0.448\end{array}$

$\mathrm{N}=19 \quad \mathrm{VO}_{2} \quad 0.025 \quad$. $\quad 0.017$

$\begin{array}{lllllll}\text { FetO }_{2} & 0.47 & 0.50 & 0.44 & 0.43 & 0.27 & 0.41\end{array}$

$\begin{array}{lll}\mathrm{VE} / \mathrm{VO}_{2} & 2.40 & 1.80\end{array}$ 


\section{Appendix 3}

Standard deviation values for Table 6.2

Subject Interval between EEG change and decrease (secs)

Y.B.

$\mathrm{N}=34$

22.72

J.B.

22.59

$\mathrm{N}=39$

J.S.

$\mathrm{N}=34$

28.73

G.O.

$\mathrm{N}=25$

31.38

K.M.

$\mathrm{N}=27$

28.74 


\section{Appendix 4}

Standard deviations for Table 6.3

\begin{tabular}{lclllll} 
& \multicolumn{2}{c}{ VE } & \multicolumn{2}{c}{ Vt } & \multicolumn{2}{c}{ Cycle Duration } \\
Subject & $\begin{array}{l}\text { Awake } \\
\text { Theta }\end{array}$ & $\begin{array}{l}\text { Theta } \\
\text { Stage 2 }\end{array}$ & $\begin{array}{l}\text { Awake } \\
\text { Theta }\end{array}$ & $\begin{array}{l}\text { Theta } \\
\text { Stage } 2\end{array}$ & $\begin{array}{l}\text { Awake } \\
\text { Theta }\end{array}$ & $\begin{array}{l}\text { Theta } \\
\text { Stage 2 }\end{array}$ \\
Y.B. & -0.46 & 0.09 & -0.33 & 0.01 & 0.46 & -0.13 \\
$\begin{array}{l}\text { N=34 } \\
\text { J.B. }\end{array}$ & -0.46 & 0.13 & -0.42 & 0.03 & -0.01 & -0.21 \\
N=39 & & & $\ddots$ & & & \\
J.S. & 0.22 & 0.21 & 0.23 & 0.20 & 0.32 & 0.21 \\
N=34 &. & & & & & \\
$\begin{array}{l}\text { G.O. } \\
\text { N=25 }\end{array}$ & 0.20 & 0.17 & 0.14 & 0.10 & 0.26 & 0.15 \\
K.M. & 0.25 & 0.14 & 0.21 & 0.13 & 0.24 & 0.13 \\
N=27 & & & & & &
\end{tabular}

